\title{
Grid Convergence for Turbulent Flows (Invited)
}

\author{
Boris Diskin,* \\ National Institute of Aerospace, Hampton, VA 23666 \\ James L. Thomas, ${ }^{\dagger}$ Christopher L. Rumsey, ${ }^{\ddagger}$ \\ NASA Langley Research Center, Hampton, VA 23681 \\ Axel Schwöppe ${ }^{\S}$ \\ DLR, Braunschweig, Germany
}

\begin{abstract}
A detailed grid convergence study has been conducted to establish accurate reference solutions corresponding to the one-equation linear eddy-viscosity Spalart-Allmaras turbulence model for two dimensional turbulent flows around the NACA 0012 airfoil and a flat plate. The study involved three widely used codes, CFL3D (NASA), FUN3D (NASA), and TAU (DLR), and families of uniformly refined structured grids that differ in the grid density patterns. Solutions computed by different codes on different grid families appear to converge to the same continuous limit, but exhibit different convergence characteristics. The grid resolution in the vicinity of geometric singularities, such as a sharp trailing edge, is found to be the major factor affecting accuracy and convergence of discrete solutions, more prominent than differences in discretization schemes and/or grid elements. The results reported for these relatively simple turbulent flows demonstrate that CFL3D, FUN3D, and TAU solutions are very accurate on the finest grids used in the study, but even those grids are not sufficient to conclusively establish an asymptotic convergence order.
\end{abstract}

\footnotetext{
* NIA Research Fellow and Research Associate Professor, MAE Department, University of Virginia, Charlottesville, VA, Associate Fellow AIAA.

${ }^{\dagger}$ Distinguished Research Associate, Computational AeroSciences Branch, Fellow AIAA.

¥Senior Research Scientist, Computational AeroSciences Branch, Fellow AIAA.

$\S$ Research Engineer, Institute of Aerodynamics and Flow Technology.
} 


\section{Nomenclature}

$\begin{array}{llll}A R_{L E} & \text { Aspect ratio at leading edge } & k & \text { Mesh gradation coefficient } \\ C_{f, x} & \text { Skin friction coefficient component } & \hat{r} & \text { Distance from reentrant corner tip } \\ C_{p} & \text { Pressure coefficient } & p & \text { Convergence order } \\ C_{L} & \text { Lift coefficient } & q & \text { Exact solution of the Laplace equation } \\ C_{M} & \text { Pitching moment coefficient } & s & \text { Size of reentrant-corner cell } \\ C_{D} & \text { Total drag coefficient } & u, w & \text { Velocity components } \\ C_{D p} & \text { Pressure drag coefficient } & \hat{x}, \hat{z} & \text { Coordinates relative to reentrant corner tip } \\ C_{D v} & \text { Viscous drag coefficient } & x, y, z & \text { Orthogonal coordinates in streamwise, } \\ M_{\infty} & \text { Freestream Mach number } & & \text { spanwise, and normal directions } \\ N & \text { Number of degrees of freedom } & x_{T E}, z_{T E} & \text { Reentrant corner / trailing-edge position } \\ P_{r e f} & \text { Reference pressure } & z^{+} & \text {Nondimensional boundary layer spacing } \\ P_{t} & \text { Total pressure } & \alpha & \text { Angle of attack } \\ P r & \text { Prandtl number } & \hat{\alpha} & \text { Inverse relative exterior angle } \\ P r_{t} & \text { Turbulent Prandtl number } & \beta & \text { Stretching factor } \\ R e & \text { Reynolds number } & \epsilon & \text { Discretization error } \\ T_{r e f} & \text { Reference temperature } & \theta & \text { Polar coordinate angle } \\ T_{t} & \text { Total temperature } & \kappa & \text { MUSCL scheme parameter } \\ a_{r e f} & \text { Reference speed of sound } & \mu_{r e f} & \text { Reference eddy viscosity } \\ c & \text { Chord length } & \mu_{t} & \text { Eddy viscosity } \\ h_{\text {avg }} & \text { Averaged mesh spacing } & \nu_{\infty} & \text { Freestream kinematic viscosity } \\ h, h_{e f f} & \text { Characteristic mesh size } & \xi & \text { Mapping coordinate } \\ h_{m a x} & \text { Maximum mesh size } & \omega & \text { External angle for reentrant corner }\end{array}$

\section{Introduction}

With ever-increasing computing power and recent advancements in solver technology, turbulent flows are routinely simulated on high-density grids with many millions of degrees of freedom. While accurate and reliable Computational Fluid Dynamics (CFD) analysis of attached turbulent flows is now possible, accuracy and robustness of separated turbulent flow simulations in complex geometries are still not adequate. Errors in such simulations are typically attributed to three sources. (1) The modeling error is due to approximations in the continuous formulation (e.g., in differential equations describing turbulent flows, or in geometry definitions, or in boundary conditions) and is defined as the difference between the exact continuous solution of the model formulation and the real flow. (2) The discretization error is due to approximations in discretizing the continuous formulation on a specific grid and is defined as the difference between the exact discrete and continuous solutions. (3) The iterative (algebraic) error is due to an imperfect iterative solution process for the discrete formulation and is defined as the difference between the exact and approximate solutions to the discrete formulation.

A Turbulence Modeling Resource (TMR) website ${ }^{1,2}$ has been recently established at NASA Langley Research Center to describe, standardize, verify, and validate formulations of common turbulence models. The purpose of this website is to avoid ambiguity associated with specific implementations of turbulence models in CFD codes. The turbulent flows considered in this paper are modeled by Reynolds-averaged Navier-Stokes (RANS) equations and the one-equation linear eddy-viscosity Spalart-Allmaras (SA) turbulence model. ${ }^{3}$ The formulation details are available at the TMR website.

This paper reports on an attempt to eliminate (or at least minimize) the discretization and iterative errors by conducting an extensive grid convergence study for relatively simple benchmark turbulent flows. Current guidelines for grid convergence studies ${ }^{4,5}$ emphasize a parametric similarity of grids forming a family and an asymptotic convergence order, which is expected to be observed on three-to-four fine grids in a family. The mesh resolution required for establishing a convergence order is sought through a uniform grid refinement. For structured grids, a family of uniformly refined grids is typically derived recursively, starting from the finest grid. Each coarser grid in the family is derived from the preceding finer grid by removing every-other grid plane/line in each dimension. This uniform-refinement approach has rigorous mathematical foundations. However, it is also expensive because it lacks the flexibility of a local refinement, which is the basis for effective grid adaptation methods.

Ideally, solutions obtained on grids in a family would monotonically converge in grid refinement to the continuous 
solution with the same asymptotic convergence order for any solution quantity. Then, the entire solution can be extrapolated to the limit of the infinite grid refinement.

A single asymptotic convergence order characterizing the entire solution is typically observed only for model problems with smooth continuous solutions. In practical computations, an asymptotic convergence order remains elusive. Computations presented at the recent Drag Prediction Workshop (DPW-V) ${ }^{6,7}$ illustrate the difficulty in realizing asymptotic convergence order for turbulent flows. A relatively close agreement was observed between different computations in predicting the drag on a three-dimensional (3D) wing-body configuration, but the grid convergence properties were very different between solutions computed with different codes or even between same-code solutions computed on different grid families. In anticipation of second-order convergence, the values of drag were plotted versus $N^{-\frac{2}{3}}$, where $N$ is the number of degrees of freedom, but second-order convergence was not observed.

The study reported in this paper aims at computing highly accurate reference solutions for some benchmark turbulent-flow cases and at providing some guidance on accuracy variation for grid families with different mesh density patterns. The main grid-convergence test case considered is a turbulent flow around the 2D NACA 0012 airfoil at $10^{\circ}$ angle of attack. The RANS equations are solved on uniformly-refined, structured, high-density grids by three well-established CFD codes: CFL3D (NASA), FUN3D (NASA), and TAU (DLR), which use different discretization and iteration schemes. Advanced turbulent-flow solver technologies recently implemented in two of these $\operatorname{codes}^{8-10}$ provide means for minimizing effects of iterative errors. FUN3D and TAU converge all residuals, including the residual of the SA turbulence-model equation, to levels comparable with the machine zero. CFL3D converges the density residual to the level of $10^{-13}$ and the SA model residual to the level of $10^{-7}$; the corresponding aerodynamic forces converge to at least five significant digits; and the pitching moment converges to at least four significant digits.

The study began with an attempt to characterize the grid-refined solutions by using grids offered in the "Turbulence Model Validation Cases and Grids" section of the TMR website. The grid-refinement study was conducted using FUN3D solutions on the family of grids then available ${ }^{9}$ - the finest grid had about $1 \mathrm{M}$ degrees of freedom. Convergence of the lift and pitching moment coefficients did not exhibit any clear order property. The lift values increased with grid refinement and then decreased on the finest grid. The pitching moment coefficients were continually increasing with grid refinement, but showed no consistent order. A detailed inspection of the solutions on the surface of the airfoil revealed erratic convergence of the pressure coefficients near the trailing edge.

This observation motivated the current grid convergence study that involves solutions obtained with the three CFD codes on three expanded grid families. The finest grids in each family have about $15 \mathrm{M}$ degrees of freedom. The grid families differ in the trailing-edge resolution and are now available in the "Cases and Grids for Turbulence Model Numerical Analysis" section of the TMR website. Convergence sensitivities to the trailing-edge resolutions as well as to various discretization aspects, such as grid elements and the order of approximation for the turbulence-model convection term, have been considered.

Besides the NACA 0012 study, an existing flat plate test case has been extended and used to study grid convergence. FUN3D solutions have been computed on a set of structured grids. The grids are also available in the "Cases and Grids for Turbulence Model Numerical Analysis" section of the TMR website. Methodologically, the paper follows the current guidelines for grid convergence study: families of uniformly refined grids are used and convergence orders of local and global solution quantities are reported.

Additionally, the convergence degradation long-known for solutions of elliptic (pure diffusion) equations on uniformly refined grids with geometric singularities ${ }^{11}$ is revisited. Elliptic equations describe diffusion phenomena and thus apply directly to low-Reynolds number (i.e., Stokes) flows. Near surfaces, turbulent-flow solutions are expected to be similar to Stokes-flow solutions. For a sharp trailing edge, the pure-diffusion solution exhibits a square-root behavior near the trailing-edge singularity and has unbounded derivatives. The discretization error converges on uniformly refined grids with the first order in the $L_{1}$ norm and with an order of 0.5 in the $L_{\infty}$ norm. A series of structured (non-uniformly) refined grids that have a higher refinement rate near the singularity than in the rest of the domain are developed and shown to recover the convergence rate obtained for smooth flows on domains without singularities. This grid refinement strategy has not been applied to turbulent-flow computations. However, its success in application to the pure-diffusion equation provides an indication that improved resolution near geometric singularities would improve the accuracy of turbulent-flow simulations.

The material in the paper is presented in the following order. First, the CFL3D, FUN3D, and TAU codes used in the study are described in Section II, including discretization details and iterative convergence strategies. Then, a benchmark turbulent flow around the NACA 0012 airfoil is introduced in Section III together with a description of the grid families and the corresponding numerical solutions obtained with the three codes. A detailed description of the solutions and grid convergence is provided for future verification of CFD solvers. A study of solution sensitivity to the variation of discretization methods and grid elements is presented. Section IV reports on a grid convergence study 
for a turbulent flow around a finite flat-plate configuration. Finally, concluding remarks are offered. The appendix considers effects of geometric singularity on accuracy for solutions of a pure-diffusion equation and presents some strategies to overcome the convergence degradation.

\section{CFD Codes Used in the Study}

This section introduces the three well-established large-scale practical CFD codes used in the grid-convergence study for the NACA 0012 airfoil. The codes represent the state of the art in aerodynamic computations.

\section{A. CFL3D}

CFL3D is a structured-grid multi-block cell-centered finite-volume code widely applied for analysis of complex flows. It has been used in many recent workshops involving complex turbulent flows ${ }^{12-15}$ and for computing benchmark turbulent-flow solutions at the TMR website. It uses second-order, upwind-biased spatial differencing scheme (a MUSCL scheme ${ }^{16,17}$ corresponding to $\kappa=1 / 3$ that allows a third-order accuracy in one dimension (1D)) for the convective and pressure terms, and second-order differencing for the viscous terms; it is globally second-order accurate. Roe's flux difference-splitting method is used to obtain fluxes at the cell faces. The option to model the full Navier-Stokes meanflow equations is exercised for all cases. In distinction from the other two codes that use the SA-neg scheme, ${ }^{18}$ CFL3D uses the standard SA one-equation turbulence model for this study. The negative values of the Spalart turbulence variable are not allowed; the minimum values are clipped at $10^{-12}$. First and second-order approximations for the convection term in the turbulence-model equation are available. A second-order approximation is used for the NACA 0012 case on grids of Family II (see Section III for grid family definitions). Initially, the firstorder approximation was used for all computations; however, the second-order approximation was found to make a significant difference on Family II grids (see figures on the TMR website). In this study, the first-order approximation is used for computations on grids of Family I and Family III. The turbulence-model diffusion term uses the thin-layer approximation. The iteration scheme is loosely coupled, i.e., first, the meanflow equations are advanced with the eddyviscosity fixed, then the turbulence-model equation is advanced with the meanflow solution fixed. CFL3D employs local time-step scaling, grid sequencing, and multigrid to accelerate convergence to steady state.

\section{B. FUN3D}

FUN3D is a finite-volume, node-centered, unstructured-grid RANS solver, which is also widely used for high-fidelity analysis and adjoint-based design of complex turbulent flows. ${ }^{15,19-25}$ FUN3D solves governing flow equations on mixed-element grids; the elements are tetrahedra, pyramids, prisms, and hexahedra. At median-dual control-volume faces, the inviscid fluxes are computed using an approximate Riemann solver. Roe's flux difference splitting is used in the current study. For second-order accuracy, face values are obtained by a MUSCL scheme, with unweighted least-squares gradients computed at the nodes. If grid lines are available, e.g., within boundary layers or in the wake, there is an option to use a directional gradient that exploits a 1D line-mapping along the grid lines. For this study, the MUSCL scheme coefficient is set to $\kappa=0.5$ for the meanflow equations and to $\kappa=0$ for the turbulence convection term. The viscous fluxes use full viscous stresses. For tetrahedral meshes, the viscous fluxes are discretized using the Green-Gauss cell-based gradients; this is equivalent to a Galerkin type approximation. For non-tetrahedral meshes, the edge-based gradients are combined with Green-Gauss gradients; this improves the h-ellipticity of the viscous operator. The diffusion term in the turbulence model is handled in the same fashion as the meanflow viscous terms. FUN3D uses the SA-neg variant of the SA turbulence mode ${ }^{18}$ that admits negative values for the Spalart turbulence variable. This variant was designed for improved numerical behavior. The SA-neg model is identical to the original SA model for positive values of the Spalart turbulence variable. FUN3D uses a second-order approximation for the convection term in the turbulence-model equation.

A multigrid solver is used to converge residuals. The relaxation scheme in this multigrid solver is a hierarchical nonlinear scheme. On the innermost level it uses a preconditioner based on a defect-correction method and iterates on a simplified first-order Jacobian with a pseudo-time term. One preconditioner iteration involves an implicit-line pass through the portion of the domain where implicit grid lines are defined, followed by a point-implicit sweep through the entire domain. The number of preconditioner iterations may vary for different nonlinear iterations. This variable preconditioner is wrapped with a Generalized Conjugate Residual (GCR) method to form a Jacobian-free linear solver that uses Frechet derivatives to approximate linear residuals. A nonlinear controller assesses the correction computed by the linear solver. The controller is responsible for the CFL adaptation strategy and for deciding when to update the Jacobian. As a result of this assessment, the suggested correction can be applied fully, partially, or completely 
discarded; the current Jacobian may be updated or reused in the next iteration; and the current CFL number may increase, decrease, or stay the same. In the relaxation scheme, the iterations can be tightly or loosely coupled, i.e., applied to the meanflow and turbulence equations collectively or separately. The multigrid iterations are always coupled in the sense that the meanflow and turbulent equations are solved on coarse grids and the meanflow and turbulence variables use a coarse-grid correction. Initially, the CFL number is ramped over a prescribed number of iterations, but then it automatically changes within prescribed bounds. The coarse-grid corrections are also assessed by the nonlinear controller and can be applied fully, partially, or completely discarded.

\section{TAU}

TAU is a finite-volume node-centered unstructured-grid RANS solver widely used for a broad range of aerodynamic and aero-thermodynamic problems. ${ }^{26}$ It offers coupling interfaces to other disciplines like structure and flight mechanics to allow for multidisciplinary simulations. ${ }^{27}$ A full derivative is available for adjoint-based shape optimization. TAU solves the 3D compressible time-accurate RANS equations on grids with mixed elements, including tetrahedra, pyramids, prisms, and hexahedra. Control volumes are constructed by median-dual partition. The numerical scheme is based on a second-order, finite-volume formulation. At control volume faces, the inviscid fluxes are computed using a central difference scheme with an added matrix-valued artificial viscosity. ${ }^{10}$ To deal with highly stretched meshes, a cell stretching coefficient is included into the scheme. The full viscous fluxes of the meanflow and turbulence equations are discretized using an edge-normal gradient formulation as an augmented average of the adjacent Green-Gauss cell gradients. ${ }^{28}$ Various turbulence models are available, ranging from eddy viscosity to full differential Reynolds stress models, ${ }^{29}$ including options for Large-Eddy Simulation (LES) and hybrid RANS/LES. The SA-neg model ${ }^{18}$ is used as the turbulence model in this study, and the SA model convection term is discretized using a second-order approximation.

A multigrid solver based on agglomerated coarse grids is used to converge to steady state. The baseline relaxation scheme of TAU in this multigrid solver is an implicit Lower-Upper Symmetric Gauss-Seidel (LU-SGS) scheme. ${ }^{30}$ Recently the LU-SGS scheme was embedded in a hierarchy of smoothers derived from a general implicit RungeKutta method to further improve reliability and efficiency of the solutions algorithms of TAU. ${ }^{10}$ The smoothers, e.g. a first-order preconditioned Runge-Kutta or Newton-Krylov generalized minimal residual (GMRES) methods, can be considered as simplified Newton methods. The smoothers differ in Jacobian approximations and in the solution methods used for the arising linear systems.

\section{NACA 0012 Airfoil}

A grid convergence study for a turbulent flow around the NACA 0012 airfoil is presented in this section. This test case corresponds to the NACA 0012 case in the "Cases and Grids for Turbulence Model Numerical Analysis" section of the TMR website. The goals of this study are (1) to establish an accurate reference solution that can be used for verification of CFD solutions computed with the SA turbulence model, (2) to evaluate the effects of grid resolution near a sharp trailing edge on convergence of turbulent-flow solutions, and (3) to assess sensitivity of the solutions to variations of discretization methods and grid elements.

\section{A. Flow Parameters, Boundary Conditions, and Discretization Details}

A turbulent essentially incompressible $\left(M_{\infty}=0.15\right)$ flow around the NACA 0012 airfoil at $\alpha=10^{\circ}$ angle of attack is considered. For the purposes of this study, the definition of the NACA 0012 airfoil is slightly altered from the original definition, so that the airfoil closes at $c=1$ with a sharp trailing edge. The exact definition is available at the TMR website. ${ }^{1}$ The Reynolds number computed per chord length is $R e=6 \mathrm{M}$. The computational domain and boundary conditions are sketched in Fig. 1. The farfield boundary conditions are based on inviscid characteristic methods. A noslip adiabatic wall condition is specified at the airfoil surface. FUN3D and TAU have a strong implementation of the wall boundary conditions; CFL3D has a weak implementation of the wall boundary conditions. $T_{r e f}=540^{\circ} \mathrm{R}$ is the freestream static temperature. Although a farfield point vortex boundary condition correction ${ }^{31}$ is recommended at the TMR website, the results below are presented without such a correction. This simplification facilitates comparisons with emerging high-order and mesh adaptation capabilities. ${ }^{32,33}$ The farfield value of the Spalart turbulence variable is $\tilde{\nu}_{\text {farfield }}=3 \nu_{\infty}$. The Prandtl number is taken to be constant at $\operatorname{Pr}=0.72$, and the turbulent Prandtl number is taken to be constant at $P r_{t}=0.9$. The molecular viscosity is computed using Sutherland's Law. ${ }^{34}$ 


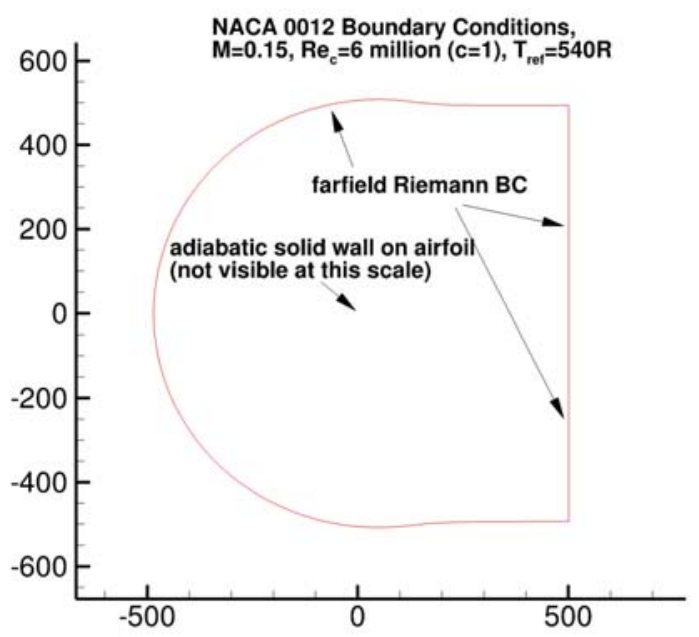

Figure 1. Domain and boundary conditions.

Table 1. NACA 0012: summary of along-surface mesh spacing on the finest 7, $169 \times 2,049$ grids in three families.

\begin{tabular}{llll} 
Grids & $\begin{array}{l}x \approx 0 \\
(\text { Leading-edge })\end{array}$ & $\begin{array}{l}x \approx 1 \\
(\text { Trailing-edge })\end{array}$ & $\begin{array}{l}x \approx 0.5 \\
(\text { Middle of the surface) }\end{array}$ \\
\hline Family I & $0.0000125 c$ & $0.0001250 c$ & $0.00123 c$ \\
Family II & $0.0000125 c$ & $0.0000125 c$ & $0.00155 c$ \\
Family III & $0.0000125 c$ & $0.0000375 c$ & $0.00139 c$
\end{tabular}

B. Grids

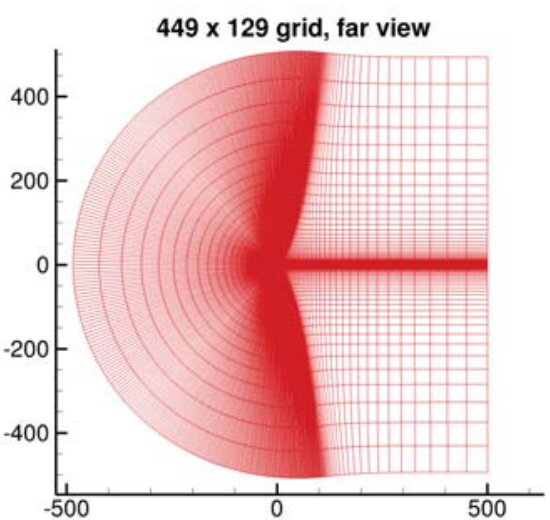

(a) Far view.

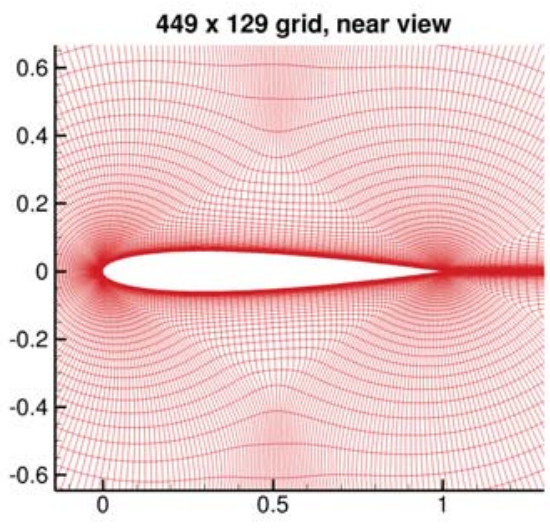

(b) Near view.

Figure 2. Computational domain and a $449 \times 129$ grid for NACA 0012 airfoil.

Three families of grids are generated with a farfield extent of approximately 500 chord lengths. Figures 2 (a) and (b) show two views of the $449 \times 129$ grid of Family I. Family I grids have the density distribution similar to the distribution used on grids of the family available on the TMR website prior to this study. Family II grids are clustered 


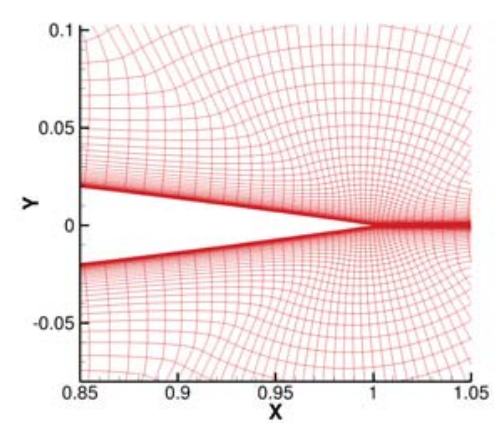

(a) Family I.

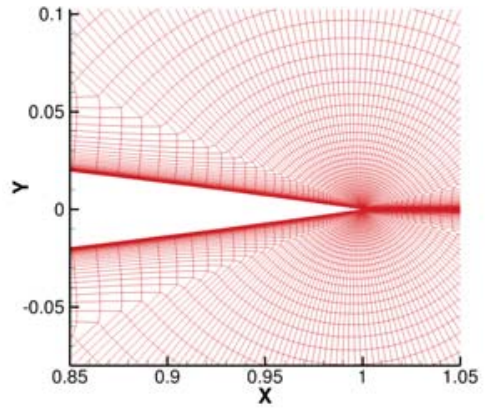

(b) Family II.

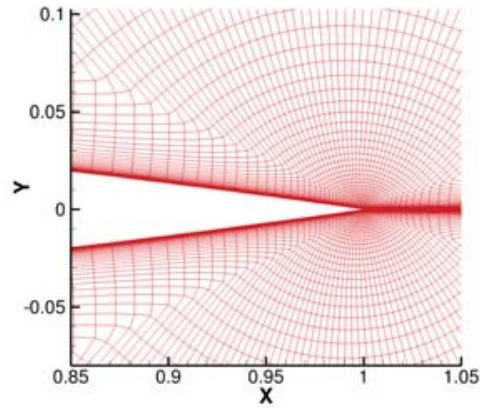

(c) Family III.

Figure 3. Near view of trailing-edge grids of Families I, II, and III.

near the trailing edge and Family III grids are intermediate between the two. See Figs. 3 (a), (b), and (c) for neartrailing-edge views of the $449 \times 129$ grids for each family. A series of seven nested grids are generated for each family, ranging from the finest 7,169 $\times 2,049$ to the coarsest $113 \times 33$ grid. The grid topology is a so-called "C $\mathrm{C}$-grid." Each of the grids wraps around the airfoil from the downstream far field, around the lower surface to the upper, then back to the downstream far field; the grid connects to itself in a one-to-one fashion in the wake. There are 4097 points on the airfoil surface on the finest grid, with 1537 points along the wake from the airfoil trailing edge to the outflow boundary. Each family's finest grid has the minimum normal spacing at the wall of $10^{-7}$. The spacing along the airfoil surface is documented in Table 1. The leading-edge spacing is the same for all families and corresponds to the aspect ratio of $A R_{L E}=125$. The trailing-edge spacing is largest for the Family I grids and ten times larger than the leading-edge spacing. On Family II grids, the trailing-edge spacing is the same as the leading-edge spacing. On Family III grids, the trailing-edge spacing is between that of Family I and Family II and three times larger than the trailing-edge spacing of the corresponding Family II grids. The family name convention is not consistent with the variation of the trailing-edge mesh spacing. The authors choose to keep the same names for grid families as in the "Cases and Grids for Turbulence Model Numerical Analysis" section of the TMR website.

The mesh spacing in the middle of the airfoil surface changes between the families. The along-surface spacings at $x \approx 0.5$ are $0.00123 c, 0.00139 c$, and $0.00155 c$ for families I, III, and II, respectively. The corresponding aspect ratios are 12300, 13900, and 15500. The relative increase in the middle-of-chord mesh spacing and aspect ratio between families I and II is approximately $25 \%$. The middle-of-chord aspect ratios are approximately two orders of magnitude higher than those at the leading edge.

\section{Grid Convergence of Aerodynamic Coefficients}

This section reports on convergence of aerodynamic coefficients on grids of families I, II, and III. Figures 4-8 compare convergence of the total drag $\left(C_{D}\right)$, pressure drag $\left(C_{D p}\right)$, viscous drag $\left(C_{D v}\right)$, lift $\left(C_{L}\right)$, and pitching moment $\left(C_{M}\right)$ with respect to the quarter-chord location. The variations are shown versus a characteristic mesh spacing $h=\sqrt{1 / N}$. FUN3D computations are shown only on the four finest grids of Family III. To accommodate a detailed scale for the $C_{L}$ and $C_{M}$ coefficients, only results on the three finest grids in each family are shown in Figs. 7-8.

Convergence plots of drag coefficients shown in Figs. 4-6 are similar on grids of different families. Convergence plots of lift and pitching moment differ significantly between grid families. Note, however, that, relatively speaking, the vertical scale for the lift figures is significantly smaller (showing variations in the fourth significant digit) than vertical scales for the drag and moment figures (showing variations in the third and first significant digits, respectively). Relatively large deviations of the CFL3D lift and moment coefficients from the corresponding FUN3D and TAU coefficients observed on Family I and Family III grids are partially explained by variations in the discretization scheme for the SA model equation. Recall that CFL3D solutions are computed with the first-order approximation for the convection term in the SA model equation on grids of Family I and Family III. Although not shown here, results on the TMR website demonstrate the effect of the SA model discretization order when using Family II grids.

All aerodynamic coefficients are predicted with a small variation between all the three codes on the finest grids of all families: the drag variation between the codes and grid families is less than 1 count (less than $1 \%$ ), the lift variation 
is less than $0.2 \%$, and the pitching moment variation is less than $10 \%$. The maximum variation is observed between the three codes on the Family I grid; the corresponding variation on the Family II grid is an order of magnitude smaller: 0.1 count (less than $0.1 \%$ ) for $C_{D}, 0.005 \%$ for $C_{L}$, and $1.5 \%$ for $C_{M}$.

The variations between the codes on finer grids within the same family are also smaller on the Family II grids than on grids of other two families. In fact, the lift and pitching moment coefficients computed on the Family I grids appear to be converging to values different from those computed on the Family II grids. This discrepancy motivated the introduction of an intermediate Family III. Family III solutions on coarser grids appear to be converging to yet another limit, but on the finest grid turn toward the values computed on the Family II grids. This behavior is observed for all codes and indicates that the solution variations due to differences in the trailing-edge resolution are larger than the variations due to differences in discretization schemes. Green dotted lines in Figs. 7-8 show the values corresponding to the infinite grid refinement computed by a linear extrapolation fitting the two finest grids. On grids of Family I, the extrapolated lift coefficients vary between values of 1.0885 and 1.0905 and the extrapolated pitching moment coefficients vary between 0.0069 and 0.0074 . On grids of Family II, the extrapolated lift coefficient is 1.0910 and the extrapolated pitching moment coefficient is 0.0068 . Note that the lift and pitching moment coefficients computed from presumably the most accurate solutions on the finest Family II grid lie outside of the range spanned by the lift and pitching moment values extrapolated from solutions on grids of Family I and Family III.

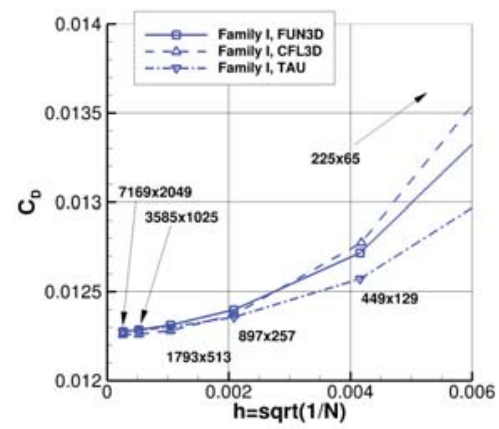

(a) Family I.

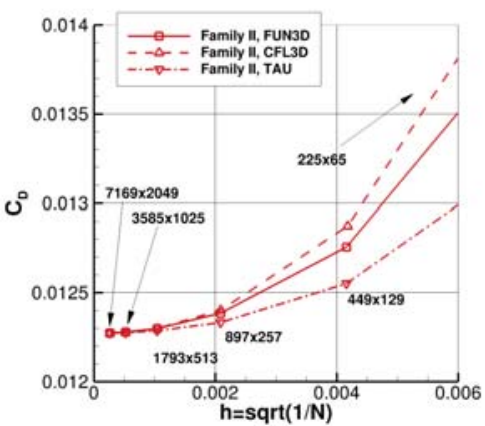

(b) Family II.

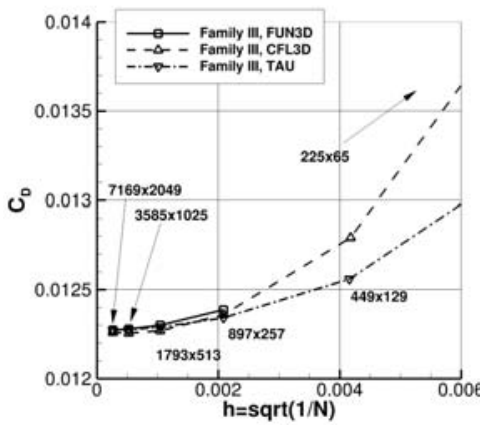

(c) Family III.

Figure 4. NACA 0012: Grid convergence of the total drag coefficient $\left(C_{D}\right)$.

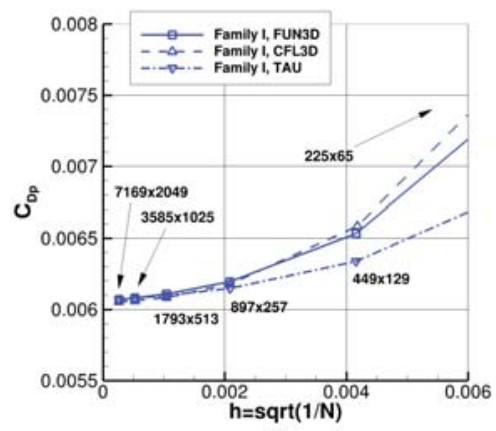

(a) Family I.

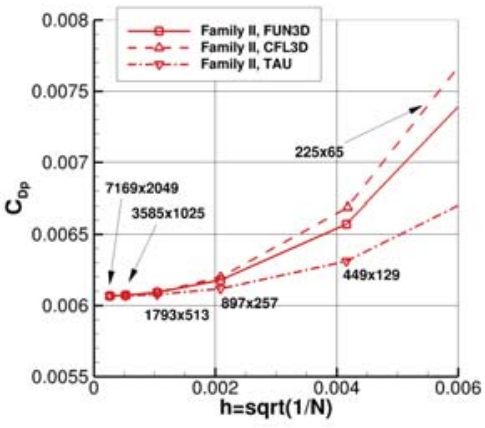

(b) Family II.

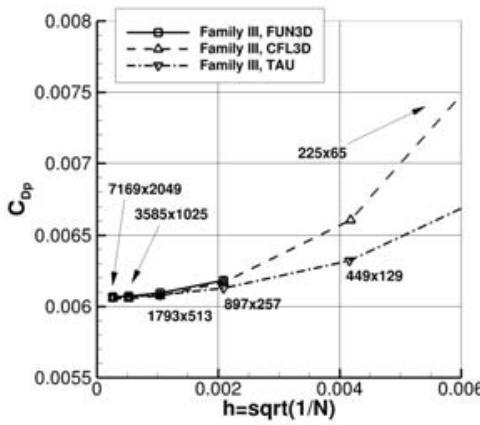

(c) Family III.

Figure 5. NACA 0012: Grid convergence of the pressure drag coefficient $\left(C_{D p}\right)$.

Figures 9-11 show variations of forces and moment computed on grids of Family II with respect to $h^{2}=1 / N$. The results are shown for forces and moment computed over the full airfoil and over the areas near the trailing and leading edges. The local integration areas are defined in Table 2. The right end of the leading-edge integration interval is selected as the $x$-coordinate of the surface node on the $897 \times 257$ grid nearest to $x=0.1$. Analogously, the left end of the trailing-edge integration interval is selected as the $x$-coordinate of the surface node on the same $897 \times 257$ grid nearest to $x=0.9$. These end nodes are present on four finer grids. The contributions to the forces and moment 


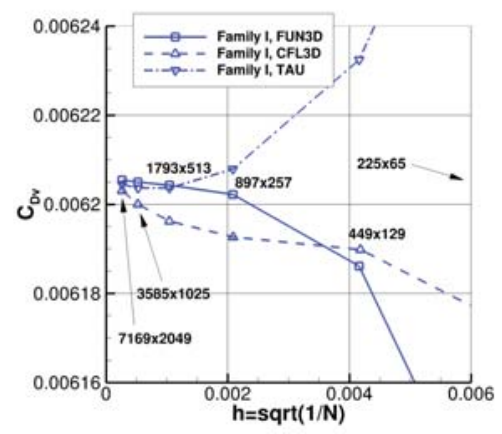

(a) Family I.

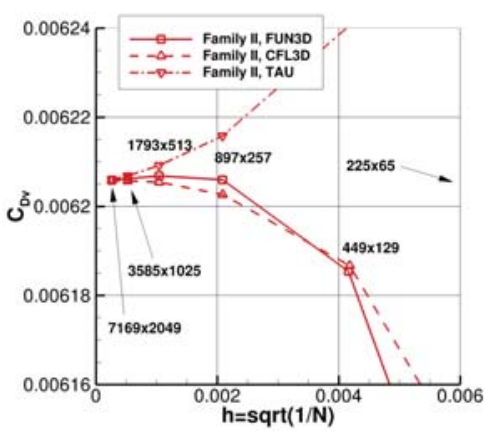

(b) Family II.

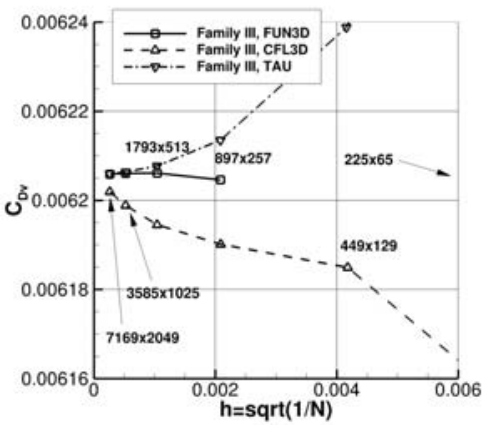

(c) Family III.

Figure 6. NACA 0012: Grid convergence of the viscous drag coefficient $\left(C_{D v}\right)$.

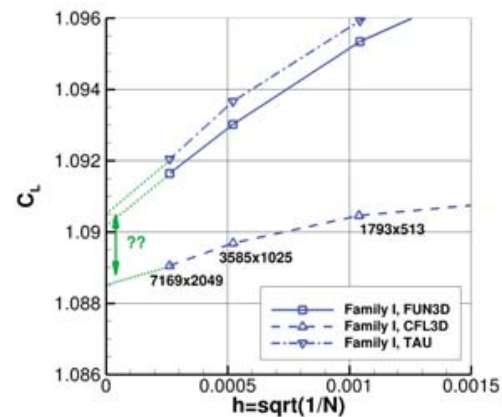

(a) Family I.

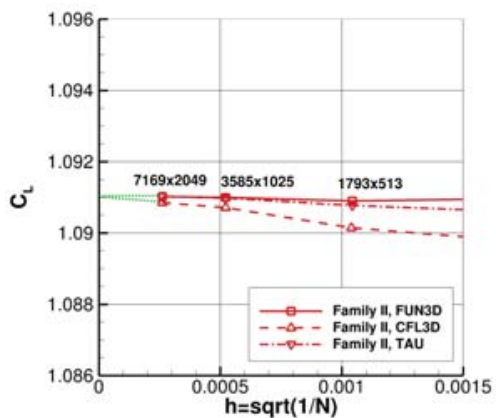

(b) Family II.

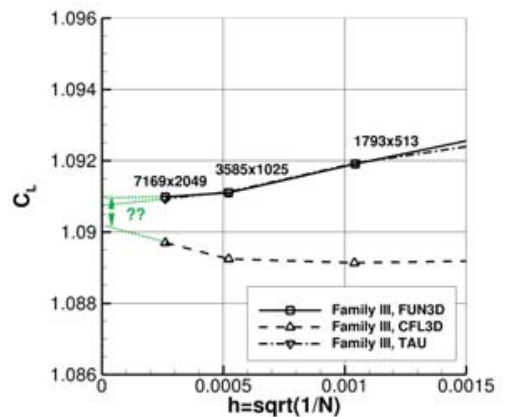

(c) Family III.

Figure 7. NACA 0012: Grid convergence of the lift coefficient $\left(C_{L}\right)$.

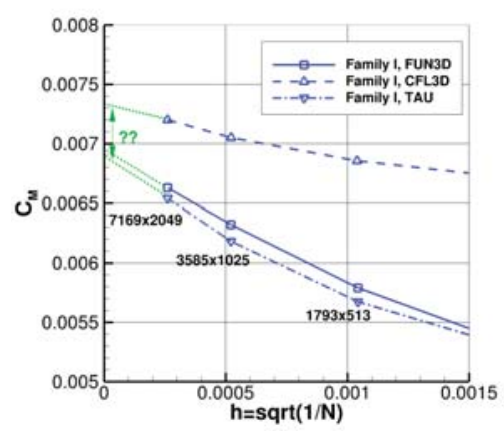

(a) Family I.

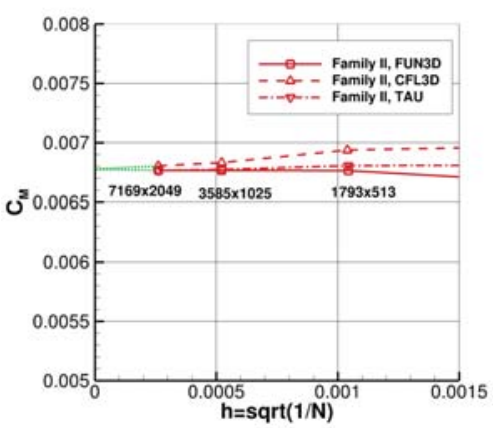

(b) Family II.

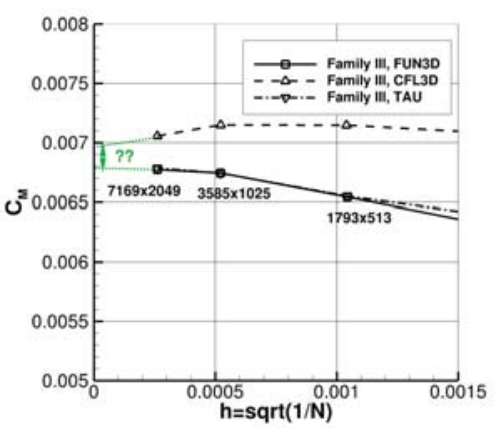

(c) Family III.

Figure 8. NACA 0012: Grid convergence of the pitching moment coefficient $\left(C_{M}\right)$.

are much larger in the leading-edge region than in the trailing-edge region. The results for CFL3D computations on quadrilateral grids and for FUN3D computations on triangular grids are plotted only for the full airfoil.

The convergence plots for lift, moment, and pressure drag are almost linear over the three finest quadrilateral grids for all three codes, indicating apparent second-order convergence. Lift and moment computed by FUN3D on 
Table 2. Leading and trailing-edge integration areas.

\begin{tabular}{cc} 
Leading Edge & Trailing Edge \\
\hline $0.0 \leq x \leq 0.100177952877727$ & $0.899166466843597 \leq x \leq 1.0$
\end{tabular}

triangular grids over the full airfoil show apparent second-order convergence over the three coarser grids and a sharp turn on the finest grid. The values of $C_{L}$ and $C_{M}$ computed by FUN3D on the finest triangular grid are close to the values computed by FUN3D and TAU on the finest quadrilateral grid. The $C_{D p}$ coefficient computed by FUN3D on triangular grids converges with an apparent order higher than second. Convergence plots for the viscous drag show less than second-order convergence for the drag computed by FUN3D and TAU over the full airfoil and over the leading-edge area. Variations of drag in the trailing-edge area appear very small. The extrapolated, grid-refined values of aerodynamic coefficients computed with different codes are not the same. CFL3D extrapolates lift and pitching moment to values somewhat different from the values extrapolated by FUN3D and TAU. These discrepancies may be a result of differences in implementation of the SA turbulence model. CFL3D employs a thin-layer approximation for the diffusion term and a standard SA formulation that does not allow negative values for the turbulence variables; FUN3D and TAU use a full-diffusion approximation and the SA-neg variant of the SA model. The extrapolated values of the lift and moment in the trailing-edge area show some differences between FUN3D and TAU solutions as well.

\section{Surface Pressure and Skin Friction}

This section compares the surface pressure and skin friction coefficients from the FUN3D, CFL3D, and TAU solutions on the finest 7, $169 \times 2,049$ grid of Family II. In moderately zoomed views focused on the leading and trailing edges (Fig. 12), the solutions are indistinguishable. Only with a super zoom (Fig. 13) do some differences come to light. Figures 13 (a) and (b) compare solutions close to the minimum pressure and the maximum skin friction locations near the leading edge. The CFL3D solution shows a smaller pressure and less skin friction than the FUN3D and TAU solutions, which are indistinguishably close to each other, even on the super-zoom view. The largest differences are observed in the immediate vicinity of the trailing edge (Figs. 13 (c) and (d)); FUN3D and TAU solutions indicate a small area of a positive load, while the CFL3D solution indicates a negative load in this area. The TAU solution shows a more oscillatory surface pressure, especially on the lower surface, than other two solutions. The near-trailing-edge maximums of the lower-surface pressure in the CFL3D and TAU solutions are comparable and larger than that in the FUN3D solution. CFL3D and TAU show a small area of negative skin friction in the immediate vicinity of the trailing edge indicating flow separation; FUN3D shows no flow separation. Although not shown, FUN3D solutions on coarse grids also have some flow separation, but it goes away with grid refinement. 


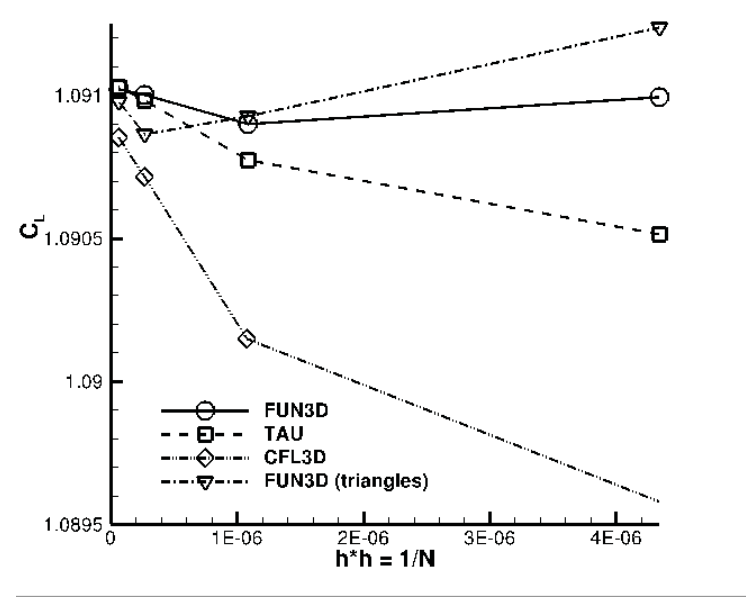

(a) Lift.

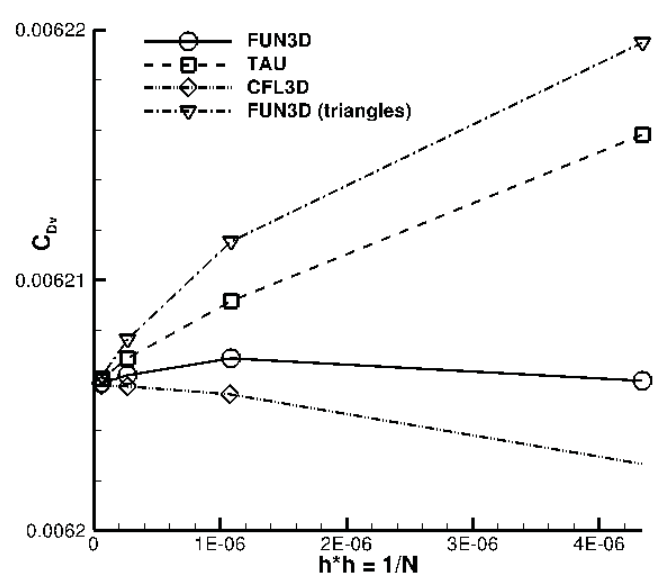

(c) Viscous drag.

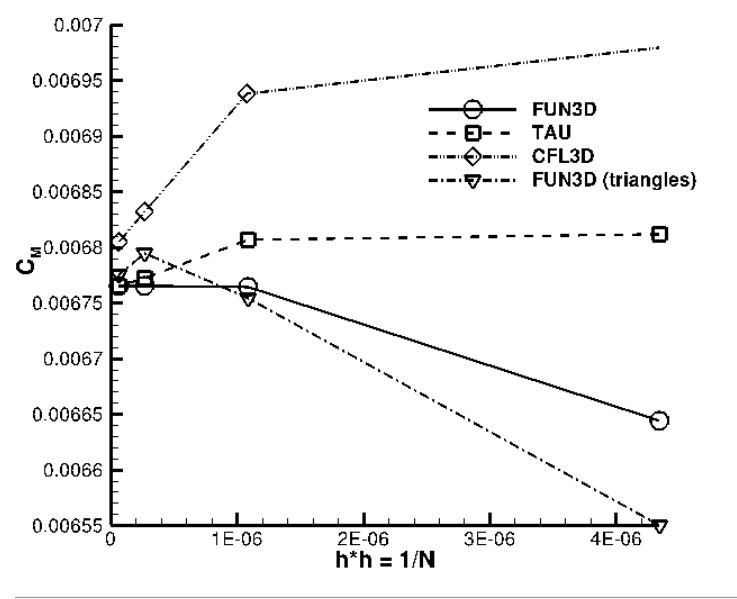

(b) Pitching moment.

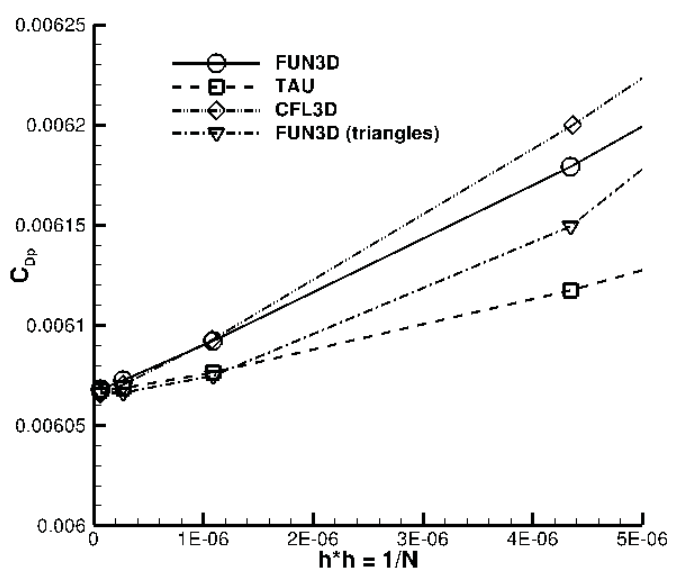

(d) Pressure drag.

Figure 9. Family II: Variation of forces and moment for the full airfoil. 


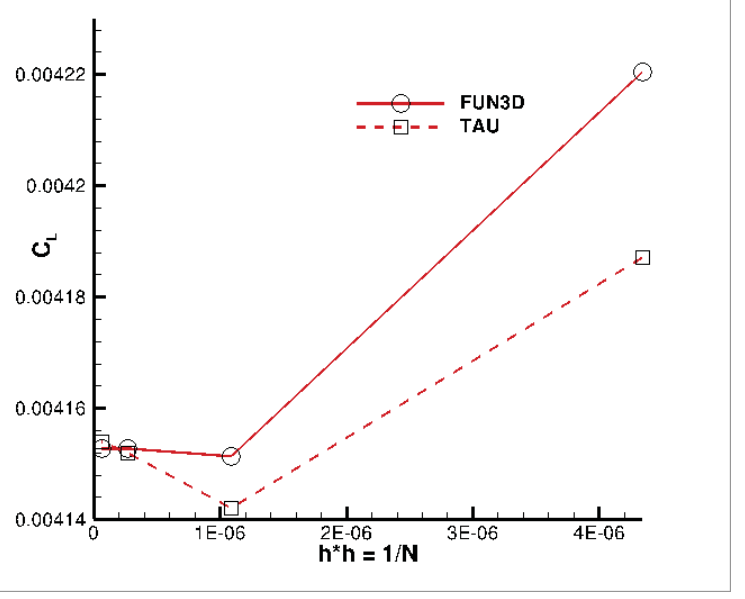

(a) Lift.

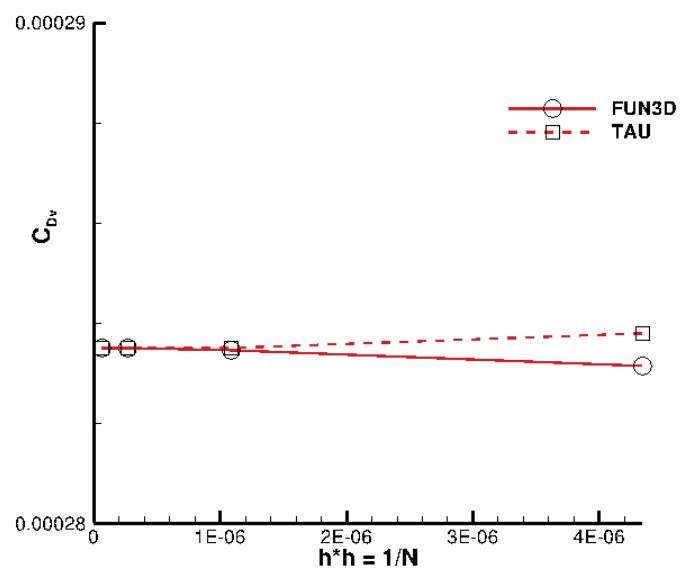

(c) Viscous drag.

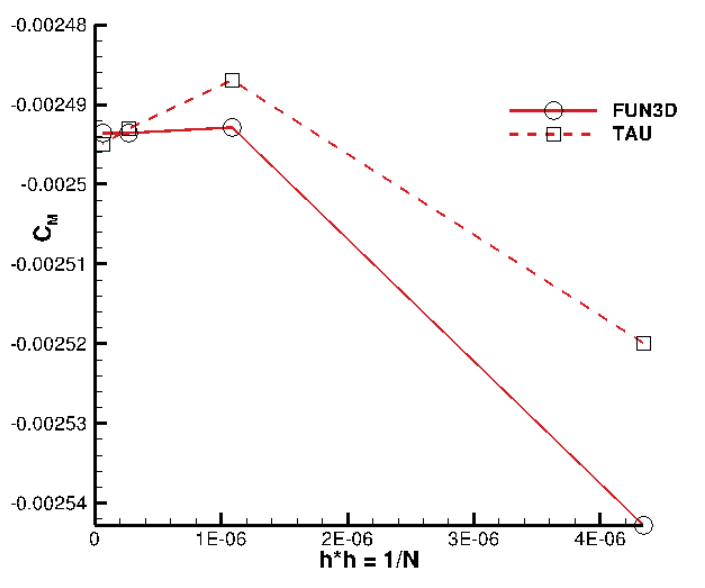

(b) Pitching moment.

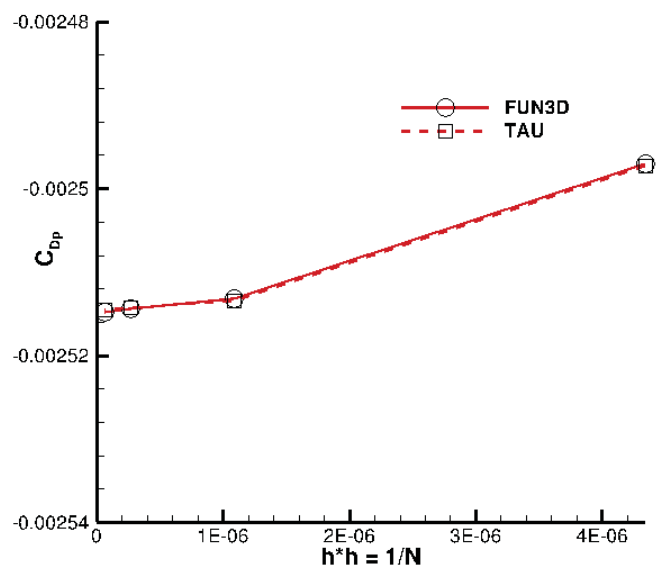

(d) Pressure drag.

Figure 10. Family II: Variation of forces and moment constrained to the trailing edge region; $0.899166466843597 \leq x \leq 1.0$. 


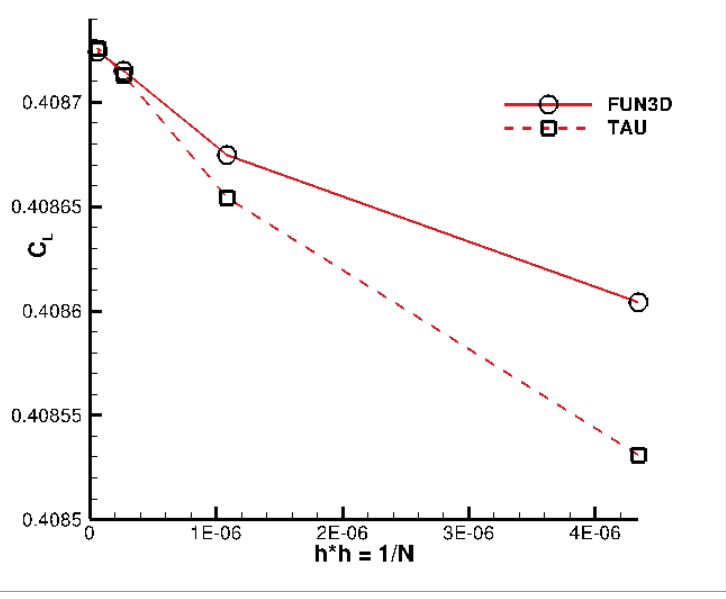

(a) Lift.

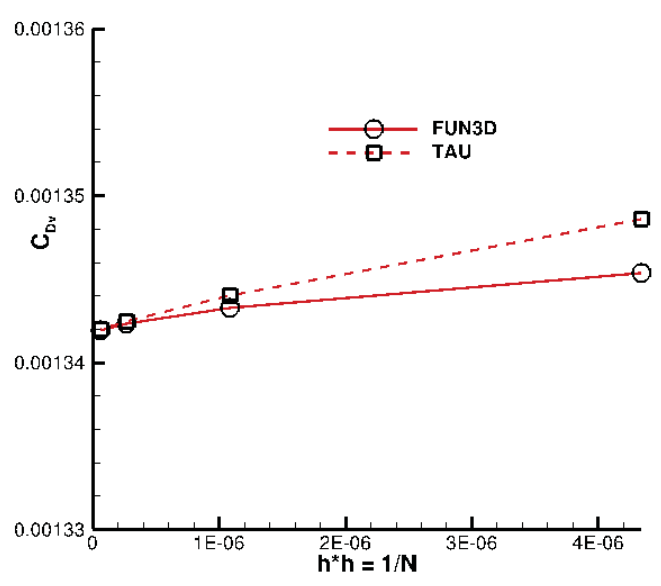

(c) Viscous drag.

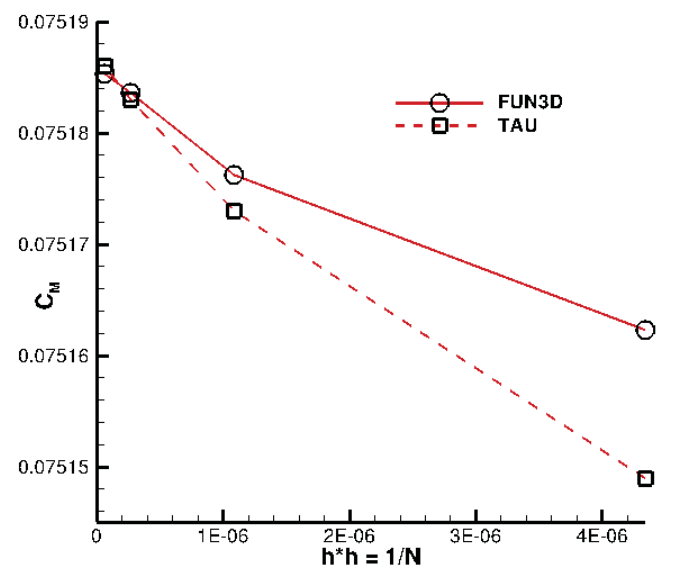

(b) Pitching moment.

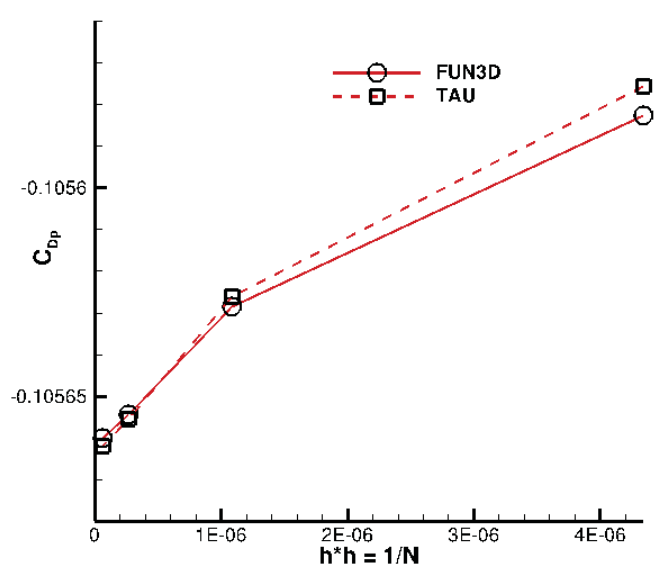

(d) Pressure drag.

Figure 11. Family II: Variation of forces and moment constrained to the leading-edge region; $0.0 \leq x \leq 0.100177952877727$. 


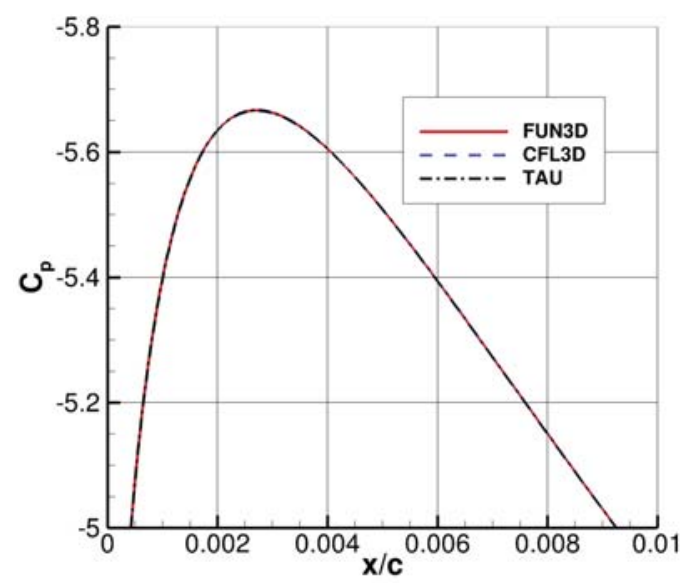

(a) $C_{P}$ at leading edge.

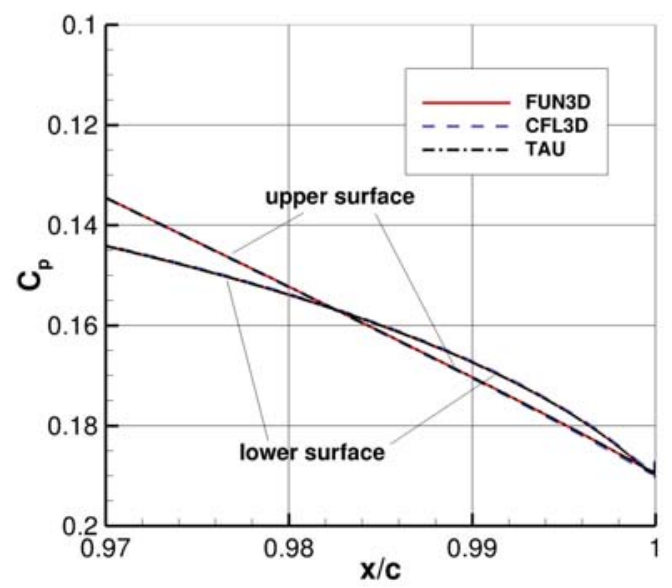

(c) $C_{P}$ at trailing edge.

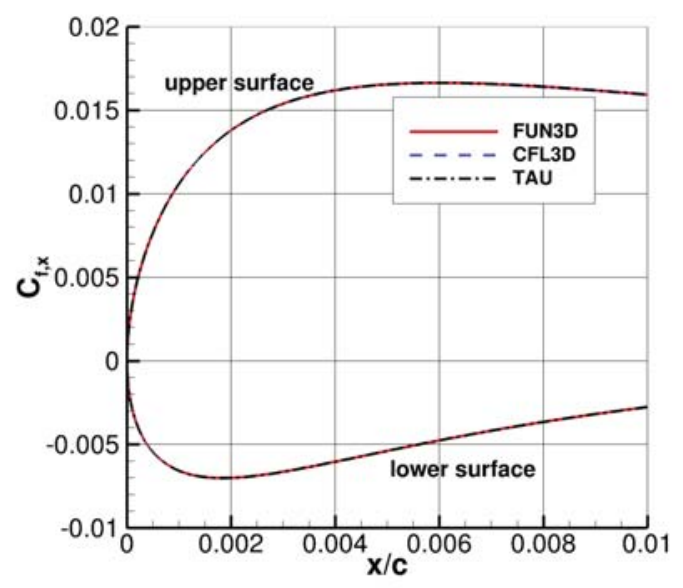

(b) $C_{f, x}$ at leading edge.

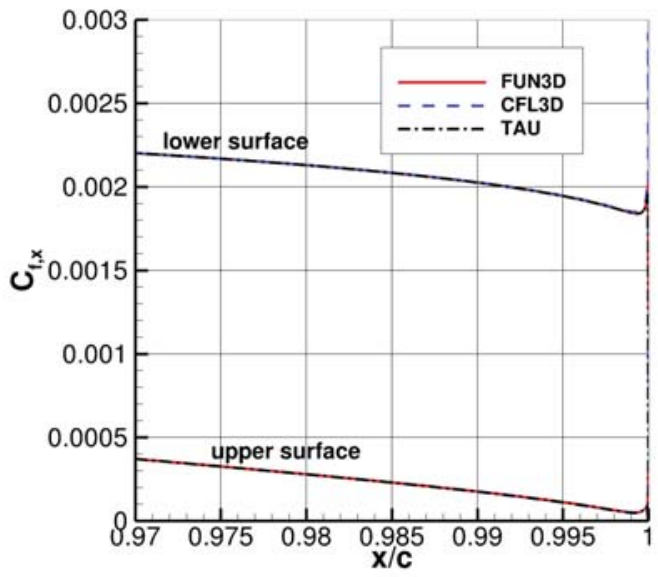

(d) $C_{f, x}$ at trailing edge.

Figure 12. Surface pressure and skin friction; moderate zoom; Family II grids. 


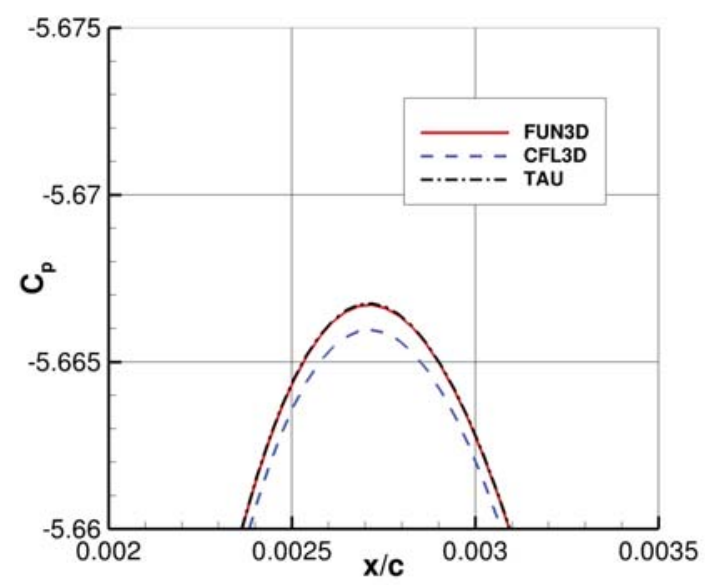

(a) $C_{P}$ at leading edge.

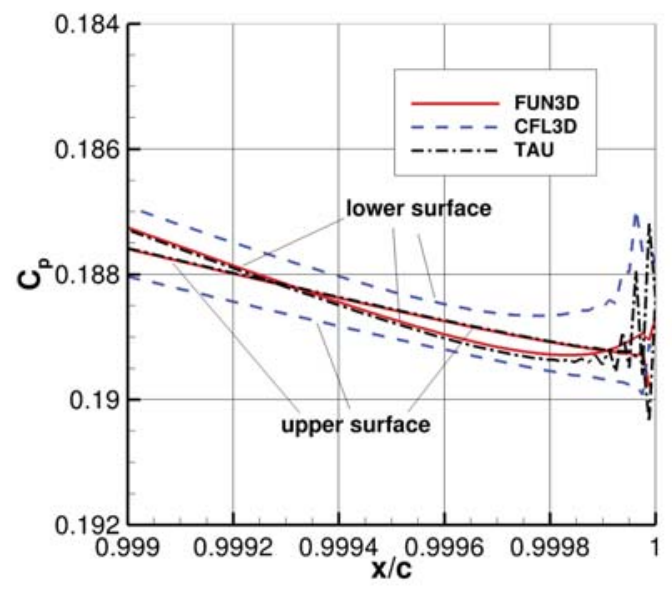

(c) $C_{P}$ at trailing edge.

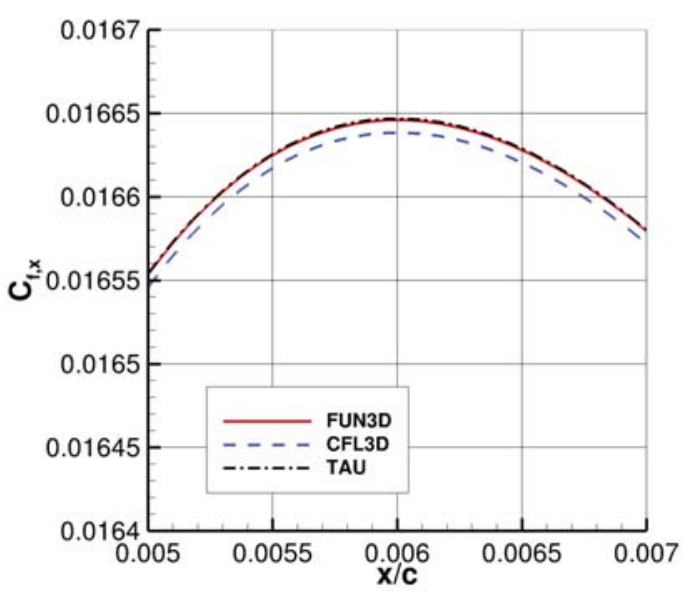

(b) $C_{f, x}$ at leading edge.

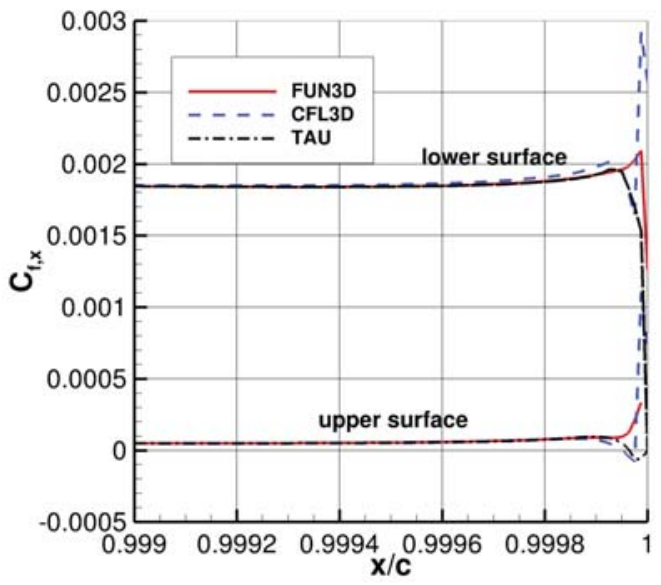

(d) $C_{f, x}$ at trailing edge.

Figure 13. Surface pressure and skin friction; super zoom; Family II grids. 


\section{E. Grid Convergence at Different Locations}

This section provides a detailed description of the reference solutions computed on Family II grids. CFL3D, FUN3D, and TAU solutions are shown at several locations near the trailing edge and in the wake. Figures exhibit convergence and variations of the pressure, velocities and the turbulence variables.

Figures 14-18 describe variations in the pressure coefficient near the trailing edge. Figure 14 shows the pressure variation along the vertical line corresponding to $x=1.001$. This is a wake location in a close proximity to the trailing edge. The solutions computed by different codes and on different grids in the family are indistinguishable. This invariance indicates that an accurate pressure profile at this location can be computed on relatively coarse grids. Figures 15 and 16 present variations along the vertical line corresponding to $x=0.999$; the variations corresponding to the upper (Fig. 15) and lower (Fig. 16) surfaces are shown separately. All solutions appear to be converging as grids are refined. CFL3D converges monotonically on the upper and lower surfaces. FUN3D and TAU converge monotonically on the upper surface. On the lower surface on the finest grid, the FUN3D solution appears to change the convergence direction. The coarse-grid TAU solution on the lower surface crosses the TAU solutions on finer grids; those finer-grid TAU solutions converge monotonically. On the upper surface, there is a noticeable difference between the CFL3D surface pressure and those of either FUN3D or TAU. The plots quickly become indistinguishable away from the surface. On the upper surface, FUN3D and TAU show larger variations in grid refinement than CFL3D solutions overall. However, the FUN3D and TAU solutions have a small variation on the 3 finest grids; the coarsest grid makes the solution variation large. On the lower surface, CFL3D shows larger variations between solutions computed on different grids than other two codes. Figures 17 and 18 show the pressure variation in the horizontal direction. Figure 17 shows variation behind the trailing edge (along the line $z=0$ ); all plots are indistinguishable. Figure 18 shows variations along the line $z=0.00008$ located slightly above the trailing edge. Even though the finest grid solutions computed by all three codes are close to each other, all codes show significant variations between solutions computed on different grids. This variability indicates that finer grid resolution is required to accurately represent the local solution.

Figures 19-21 show vertical variations of the horizontal velocity component, $u$, near the trailing edge. All plots are (almost) indistinguishable. Larger variations in $u$ are shown in Fig. 22 in the wake region along the vertical line corresponding to $x=10$. The variations are significant between solutions computed on different grids - the solutions on coarser grids do not resolve the wake velocity profile sufficiently. The variations between codes on the same grids are small. Figures 23 and 24 show variations of the $u$ velocity in the horizontal direction near the trailing edge. The plots of the wake profile along $z=0$ are indistinguishable. The variations along the $z=0.0008$ line are significant between solutions computed by different codes and on different grids. The coarse-grid solutions indicate the presence of a reverse flow near the location corresponding to $x \approx 0.9996$. The reverse-flow pocket is larger in the CFL3D solution than in the FUN3D and TAU solutions. The reverse-flow pocket disappears on the finest grid for all solutions.

Figures 25-30 demonstrate variation of the vertical velocity component, $w$, near the trailing edge and in the wake. Lines showing vertical variation near the trailing edge (Figs. 25-27) and the horizontal variation behind the trailing edge (Fig. 28) are indistinguishable. Large variations of $w$-velocity are observed between solutions on different grids in the wake region along the line $x=10$ (Fig. 29) and near the trailing edge along the line $z=0.00008$ (Figs. 30). Variations between solutions computed with different codes on the same grids are small.

Variations of the eddy viscosity near the trailing edge and in the wake are shown in Figs. 31-36. All eddyviscosity plots in the near-trailing-edge region are close to each other. There are some visible differences at the edge of the boundary layer shown in Figs. 31-33. CFL3D shows a larger variation between solutions on different grids than FUN3D. The TAU solution on each grid shows a small local oscillation of the eddy viscosity at $z \geq 0.047$ (Figs. 31 (c) and 32 (c)) and another one at $z \leq-0.017$ (Fig. 33 (c)); other codes show a smooth transition to zero in these regions. The explanation for this oscillation is that TAU uses a central difference scheme with a small matrix-valued artificial dissipation for inviscid fluxes, while the other two codes use upwind-biased schemes for inviscid fluxes. Note that the amplitude and footprint of the oscillation quickly decrease with grid refinement.

Similar to the meanflow characteristics, eddy viscosity has a significant variation in the wake (Fig. 34) and a small variation behind the trailing edge (Fig. 35). Note that CFL3D solutions on the two finest grids are not shown in Fig. 35 (a). With the SA model residuals at the level of $10^{-7}$, the CFL3D eddy-viscosity profiles along the cut $z=0$ were still changing, albeit very slowly. The computations on the two finest grids were stopped before converged eddyviscosity profiles have been achieved. The observed wake variations are mainly between eddy viscosity computed on different grids. Similar profiles are obtained by different codes on the same grids. In distinction from the meanflow characteristics, the grid variations of eddy viscosity near the trailing edge along the line $z=0.00008$ (Fig. 36) are small and plots are very similar for all solutions. Although not shown, eddy-viscosity convergence is very sensitive to the approximation order for the convection term in the SA model equation. CFL3D solutions computed with the first- 
order approximation showed a significant deterioration of accuracy and convergence for the eddy-viscosity profiles near the trailing edge and in the wake.

Figure 37 shows variations of the non-dimensional Spalart turbulence variable in FUN3D solutions. Figures 37 (a) and (b) zoom to two locations, the boundary-layer edge and the wake edge, where negative values of the turbulence variable are observed. Near the boundary-layer edge, the coarser-grid solutions show negative turbulence values of larger amplitude than fine-grid solutions. On finer grids, the area with negative turbulence variables is significantly reduced. However, the number of nodes with negative turbulence values appears approximately constant on all grids. Near the wake edge, the area of negative turbulence variables decreases on finer grids, but the number of affected nodes does not decrease. The amplitude of the negative turbulence initially increases in grid refinement, but decreases on the finest grid.

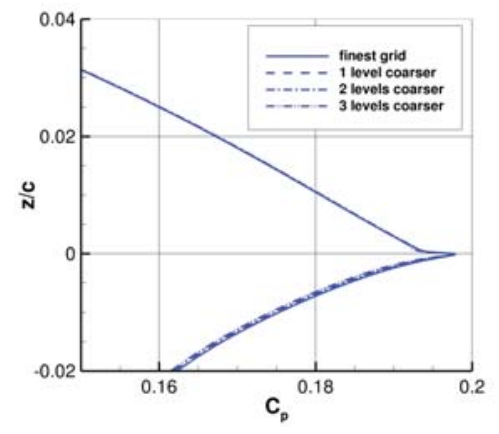

(a) CFL3D.

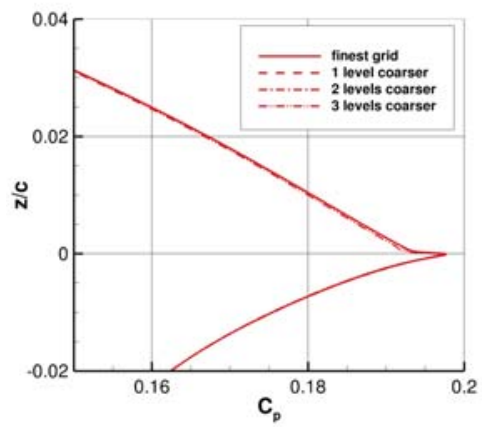

(b) FUN3D

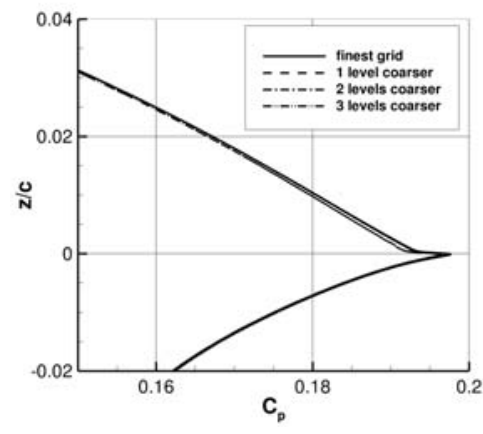

(c) TAU.

Figure 14. $C_{P}$ variation behind the trailing edge along the line $x=1.001$.

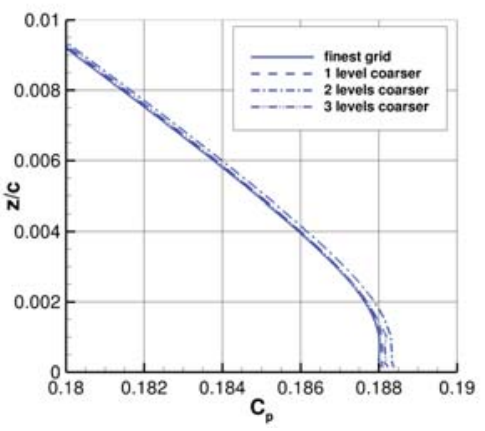

(a) CFL3D.

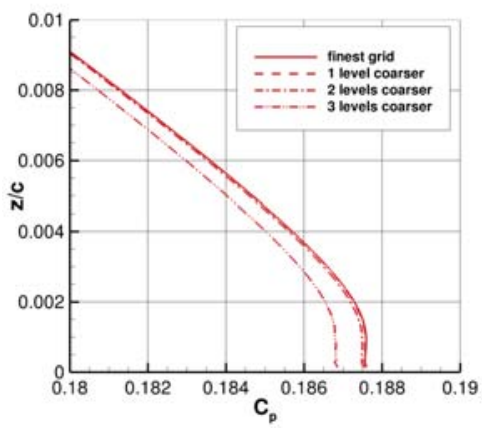

(b) FUN3D.

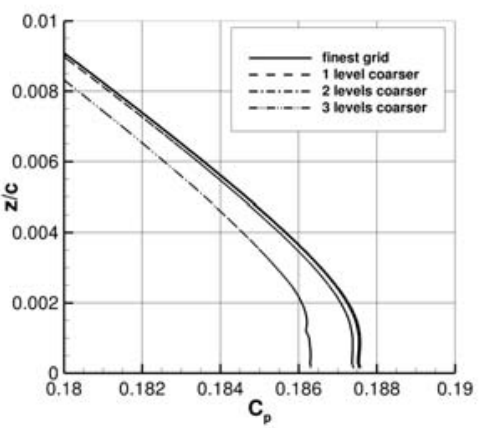

(c) TAU.

Figure 15. $C_{P}$ variation along the line $x=0.999$ over the upper surface. 


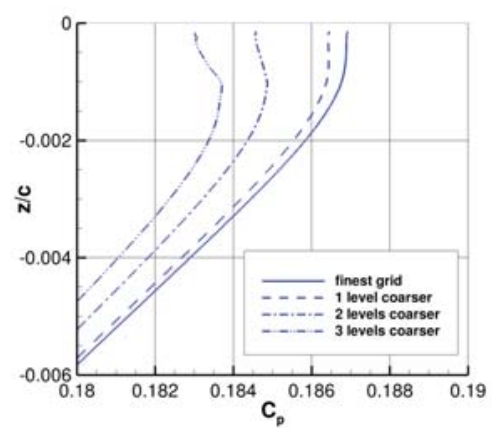

(a) CFL3D.

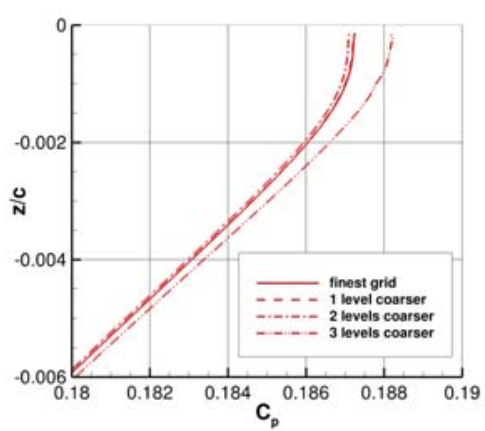

(b) FUN3D.

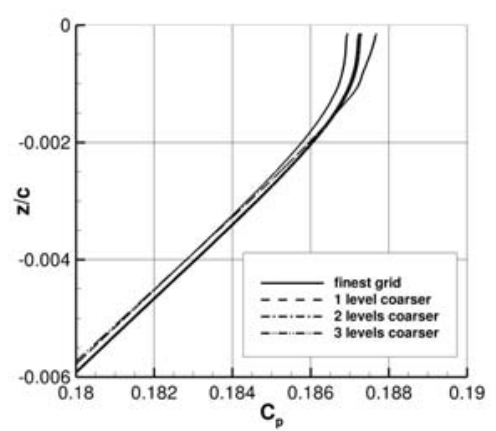

(c) TAU.

Figure 16. $C_{P}$ variation along the line $x=0.999$ under the lower surface.

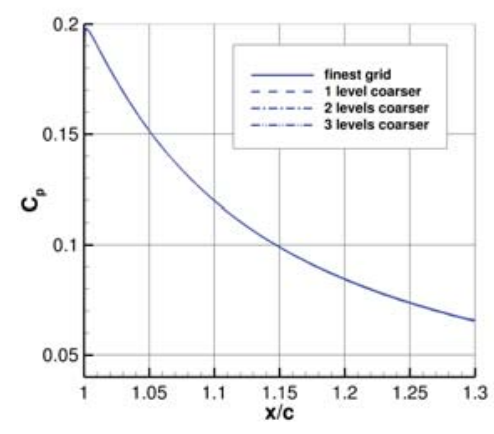

(a) CFL3D.

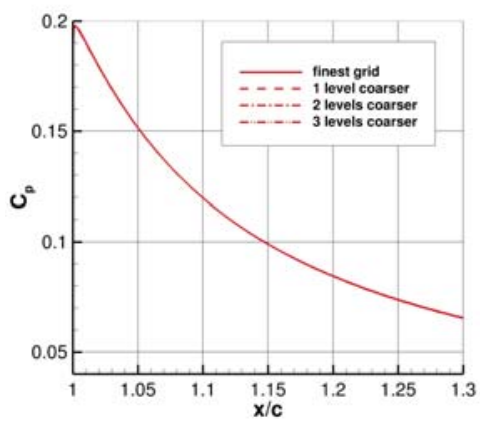

(b) FUN3D.

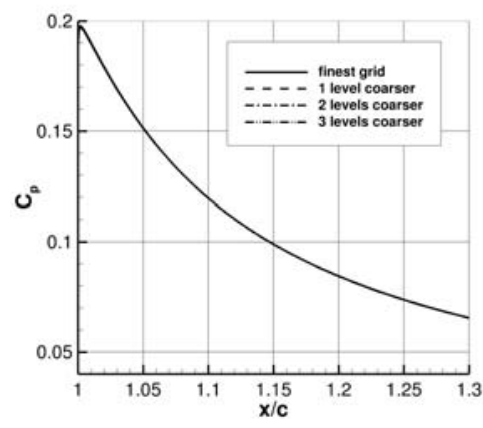

(c) TAU.

Figure 17. $C_{P}$ variation behind the trailing edge along the line $z=0$.

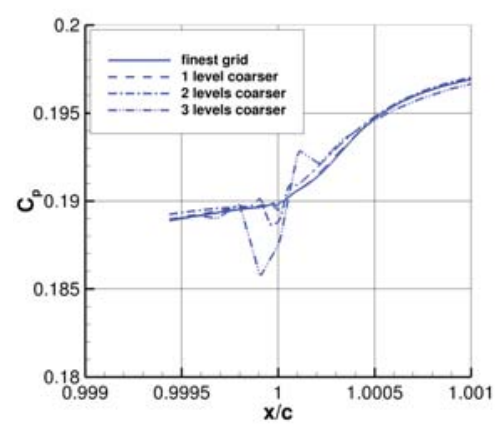

(a) CFL3D.

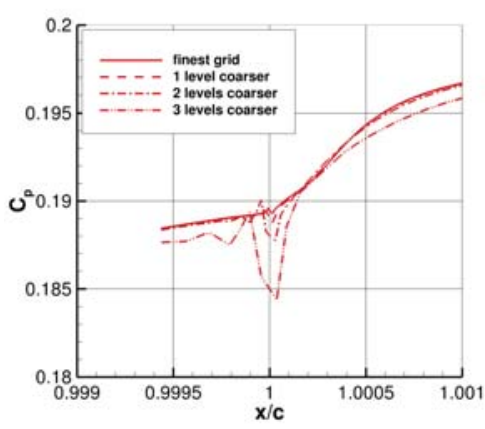

(b) FUN3D.

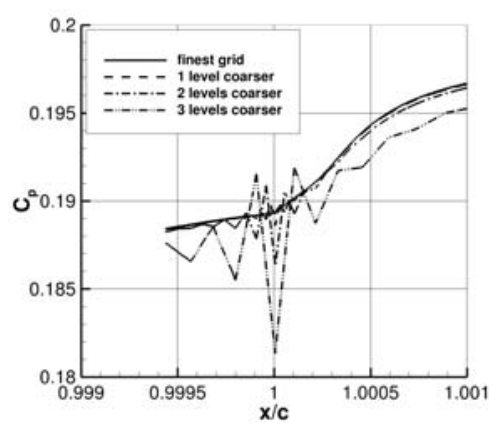

(c) TAU.

Figure 18. $C_{P}$ variation near the trailing edge along the line $z=0.00008$. 


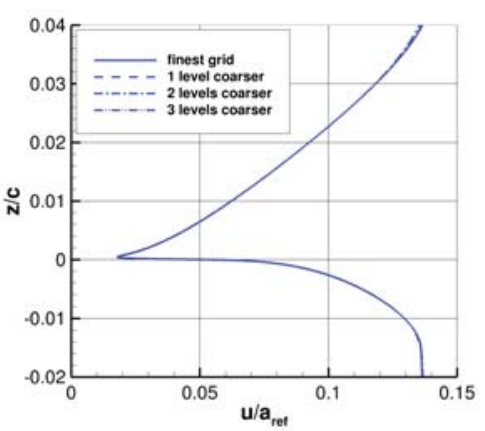

(a) CFL3D.

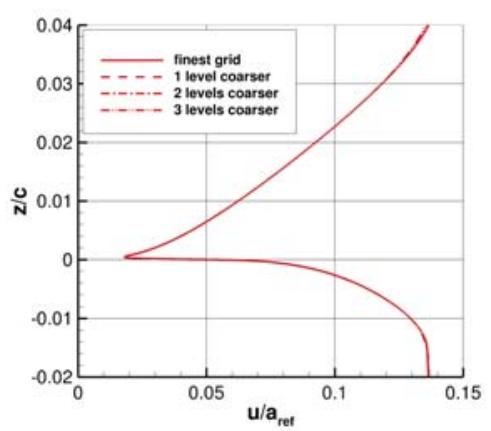

(b) FUN3D.

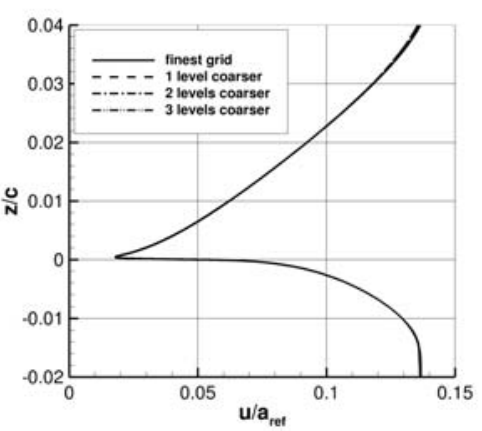

(c) TAU.

Figure 19. $u$-velocity variation behind the trailing edge along the line $x=1.001$.

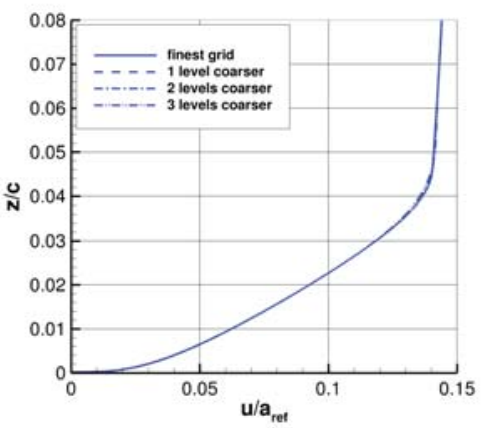

(a) CFL3D.

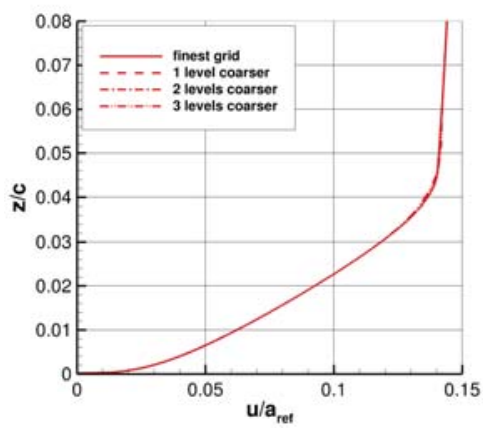

(b) FUN3D.

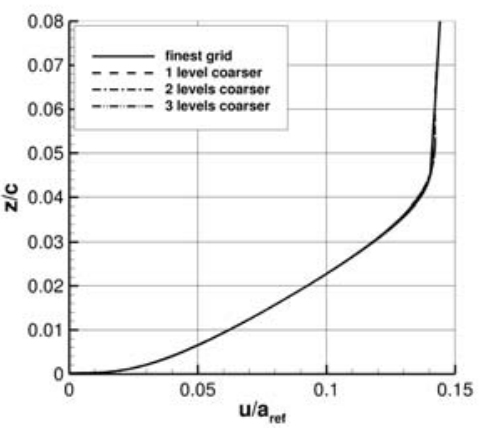

(c) TAU.

Figure 20. $u$-velocity variation along the line $x=0.999$ over the upper surface.

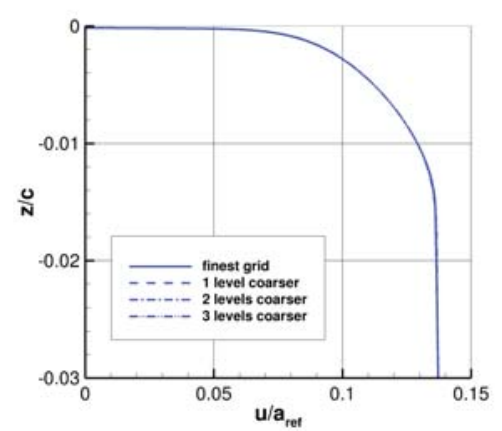

(a) CFL3D.

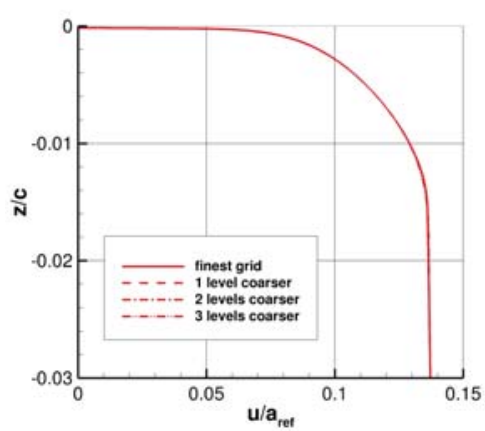

(b) FUN3D.

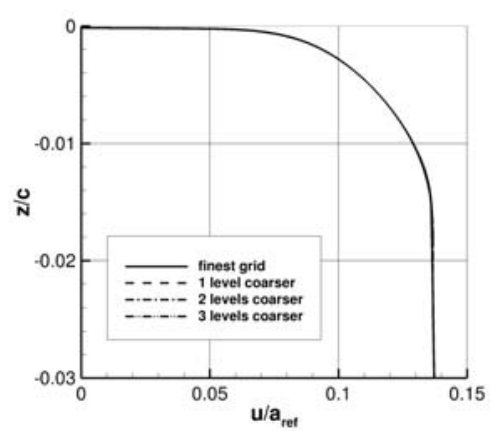

(c) TAU.

Figure 21. $u$-velocity variation along the line $x=0.999$ under the lower surface. 


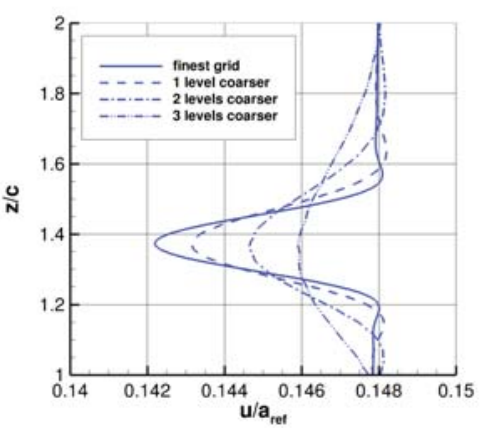

(a) CFL3D.

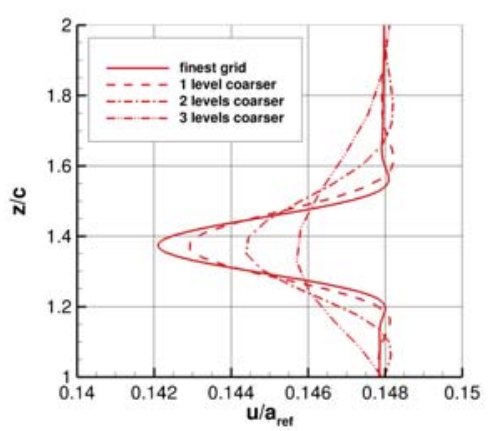

(b) FUN3D.

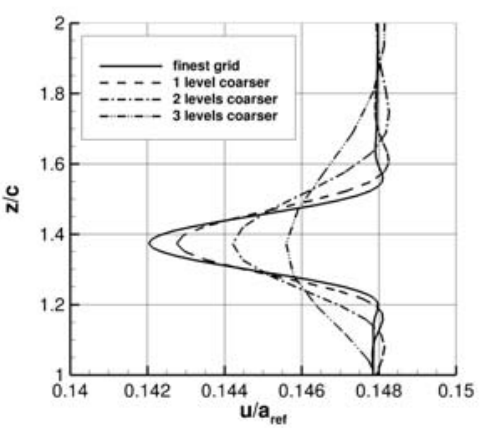

(c) TAU.

Figure 22. $u$-velocity variation in the wake along the line $x=10$.

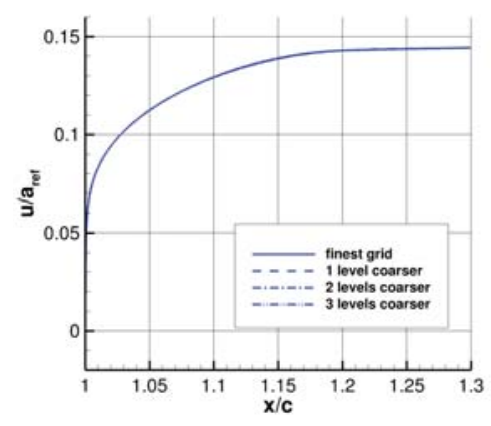

(a) CFL3D.

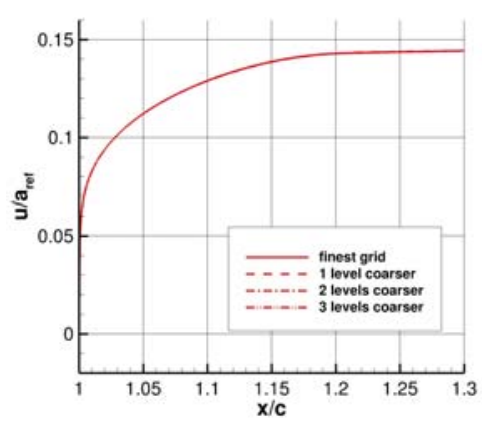

(b) FUN3D

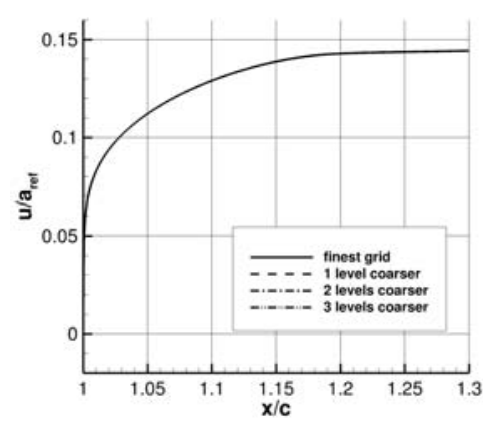

(c) TAU.

Figure 23. $u$-velocity variation behind the trailing edge along the line $z=0$.

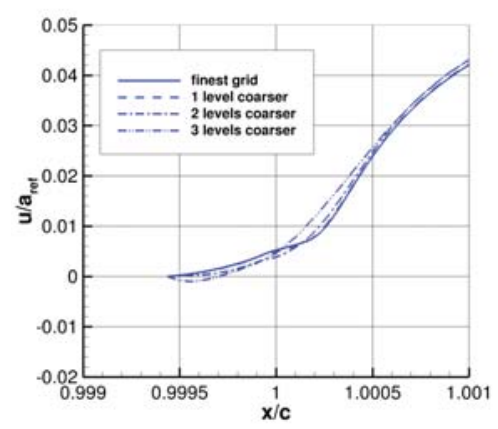

(a) CFL3D.

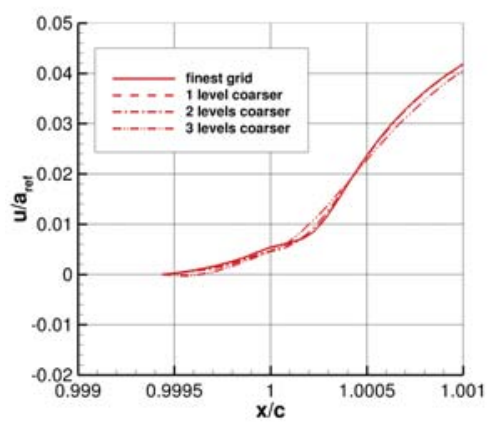

(b) FUN3D

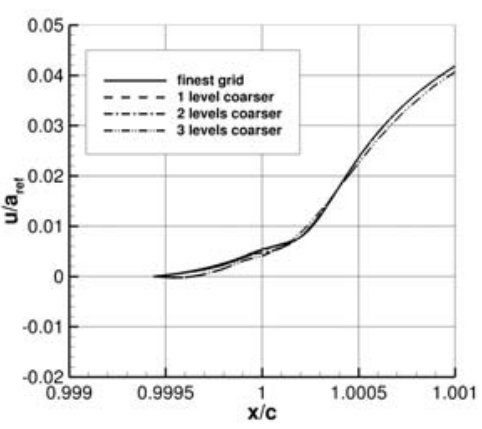

(c) TAU.

Figure 24. $u$-velocity variation near the trailing edge along the line $z=0.00008$. 


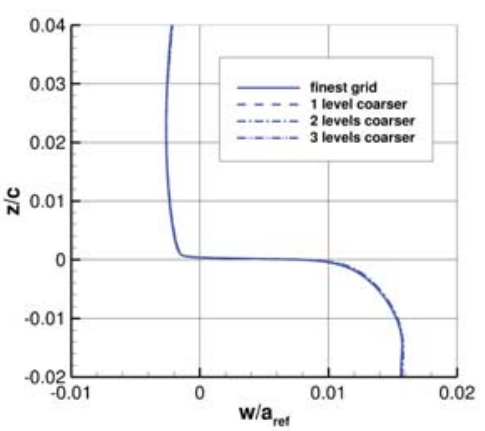

(a) CFL3D.

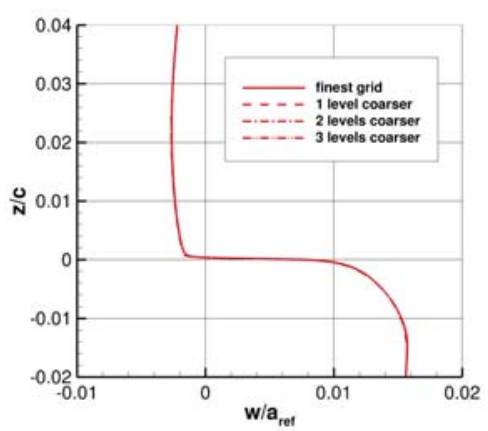

(b) FUN3D.

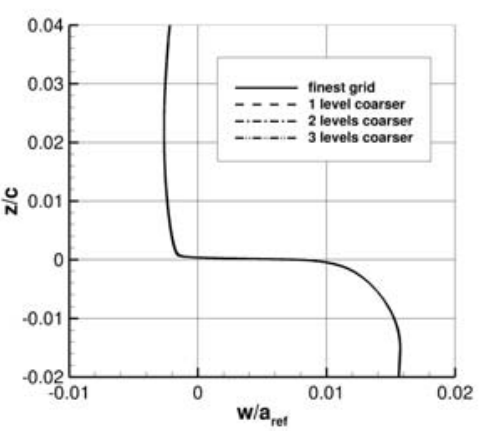

(c) TAU.

Figure 25. $w$-velocity variation behind the trailing edge along the line $x=1.001$.

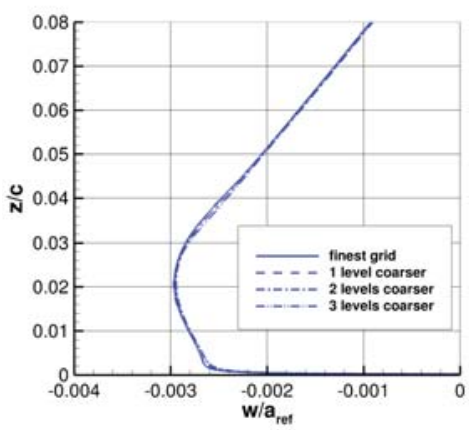

(a) CFL3D.

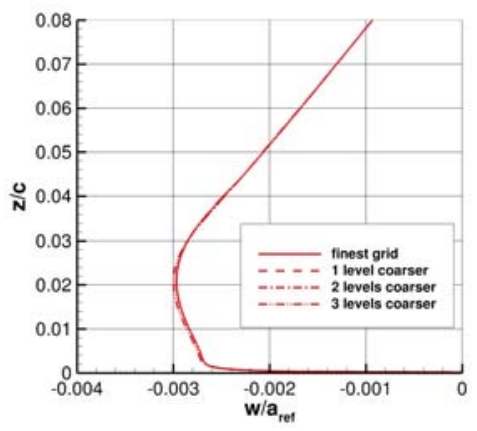

(b) FUN3D

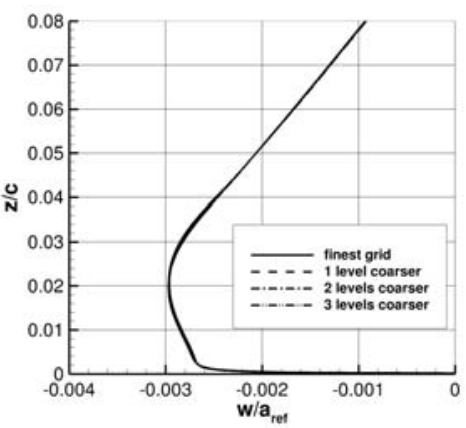

(c) TAU.

Figure 26. $w$-velocity variation along the line $x=0.999$ over the upper surface.

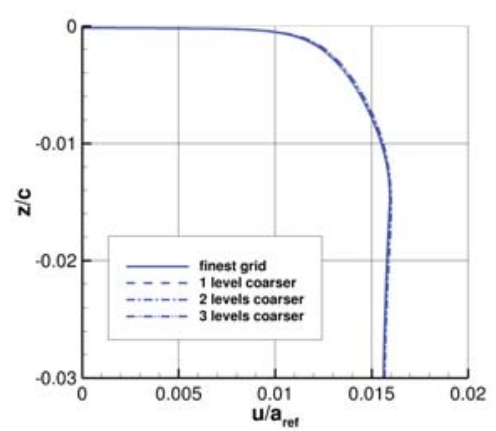

(a) CFL3D.

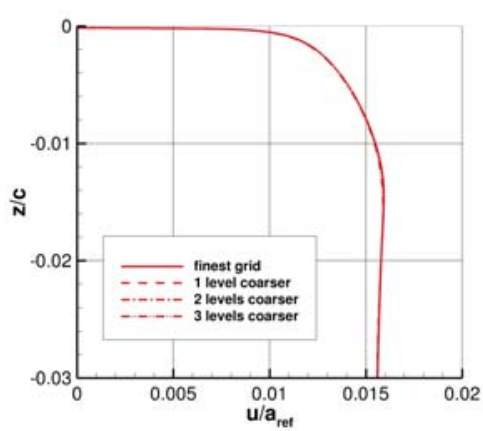

(b) FUN3D.

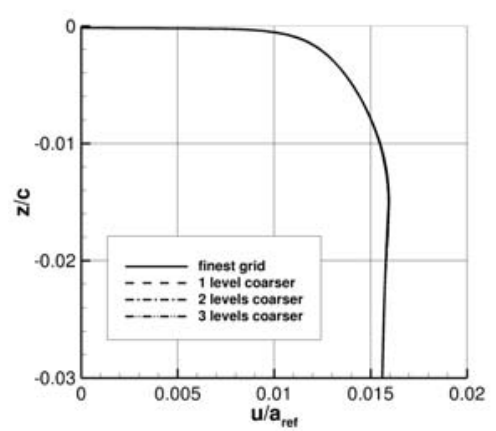

(c) TAU.

Figure 27. $w$-velocity variation along the line $x=0.999$ under the lower surface. 


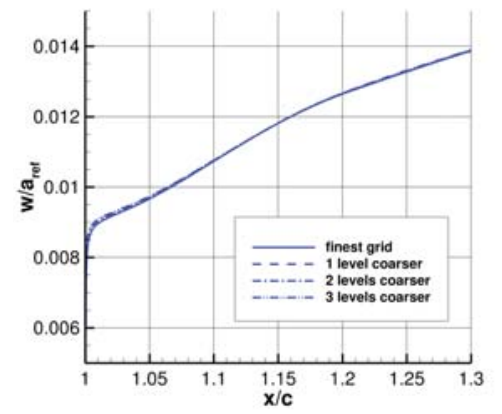

(a) CFL3D.

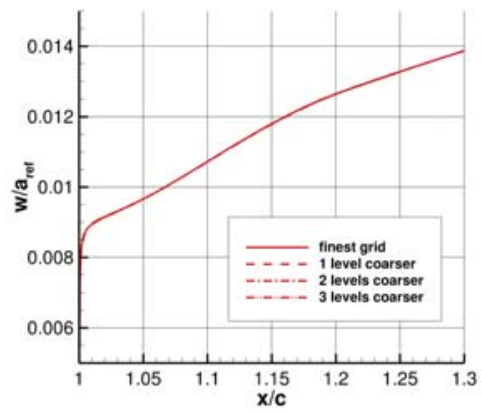

(b) FUN3D.

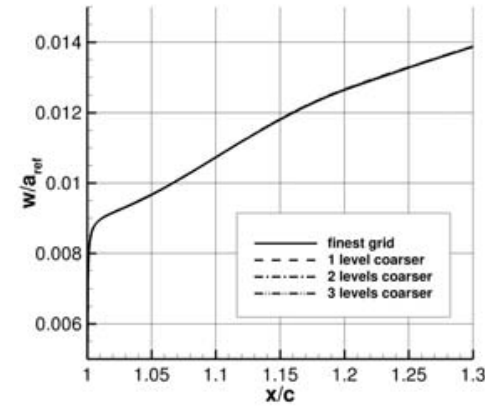

(c) TAU.

Figure 28. $w$-velocity variation behind the trailing edge along the line $z=0$.

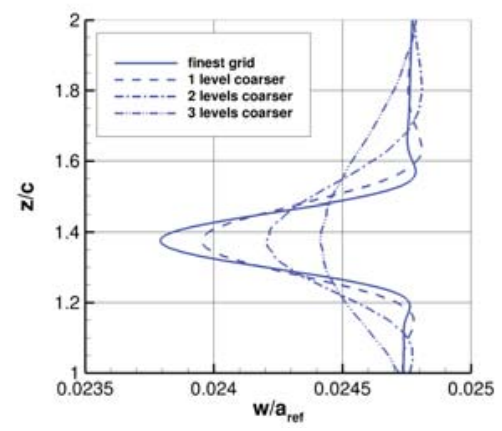

(a) CFL3D.

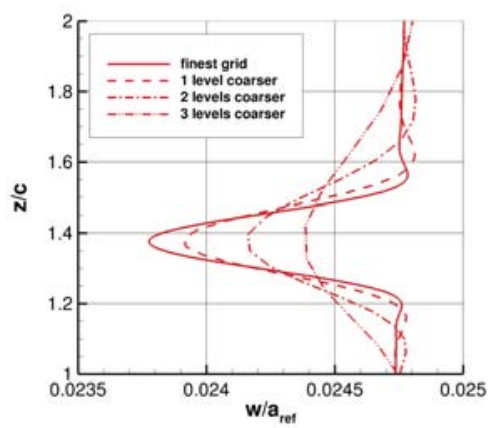

(b) FUN3D

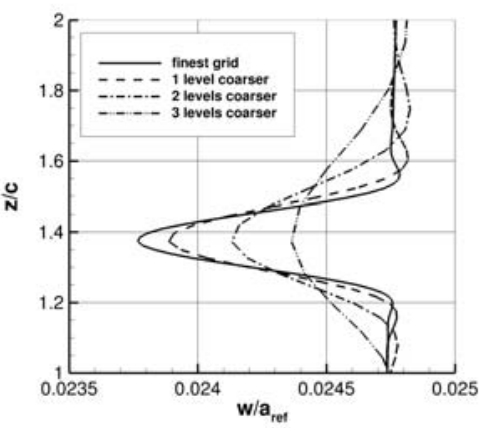

(c) TAU.

Figure 29. $w$-velocity variation in the wake along the line $x=10$.

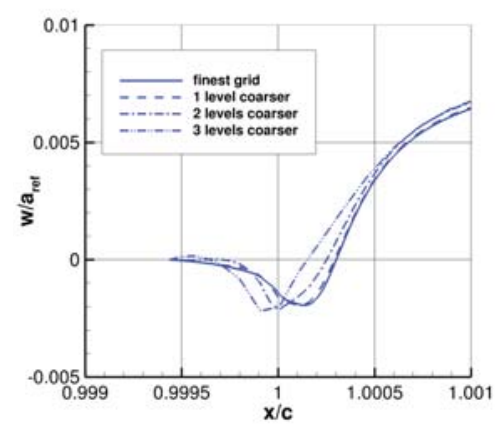

(a) CFL3D.

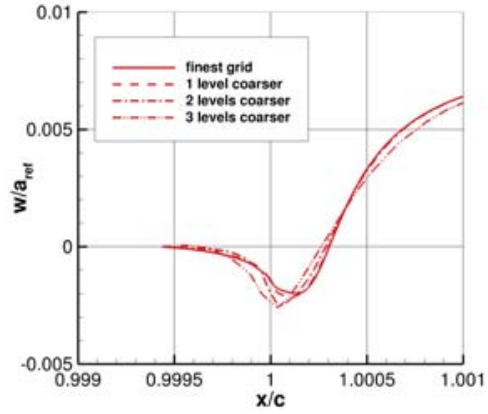

(b) FUN3D.

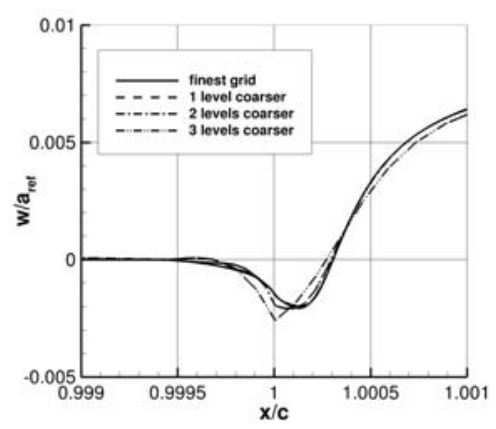

(c) TAU.

Figure 30. $w$-velocity variation near the trailing edge along the line $z=0.00008$. 


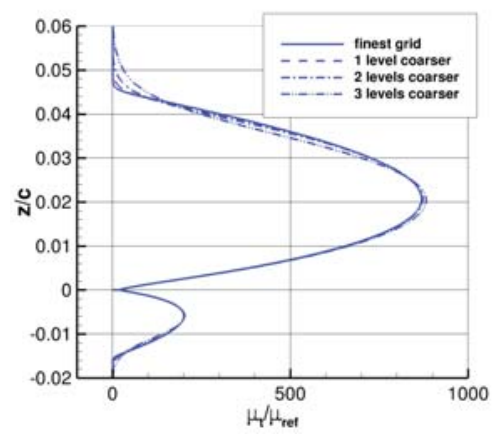

(a) CFL3D.

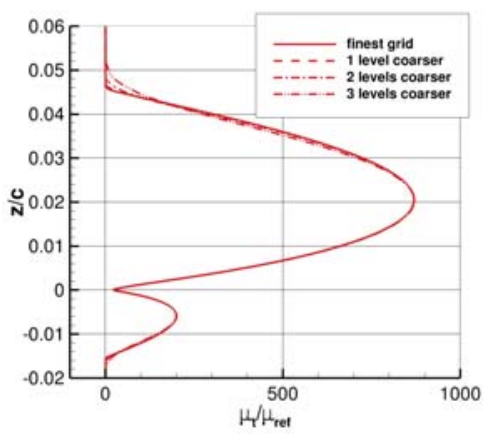

(b) FUN3D.

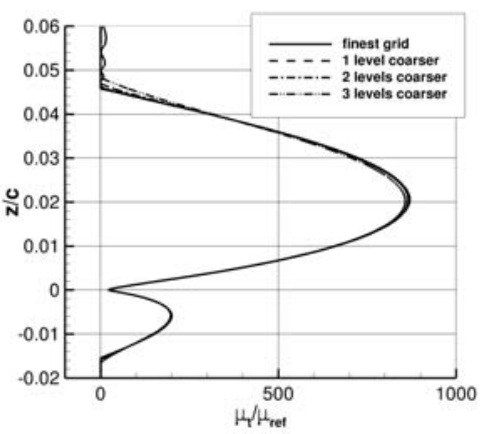

(c) TAU.

Figure 31. Eddy-viscosity variation behind the trailing edge along the line $x=1.001$.

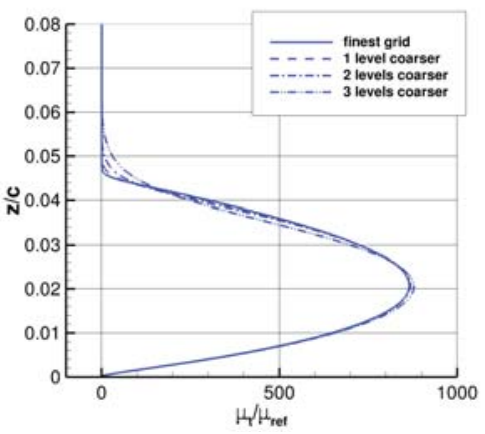

(a) CFL3D.

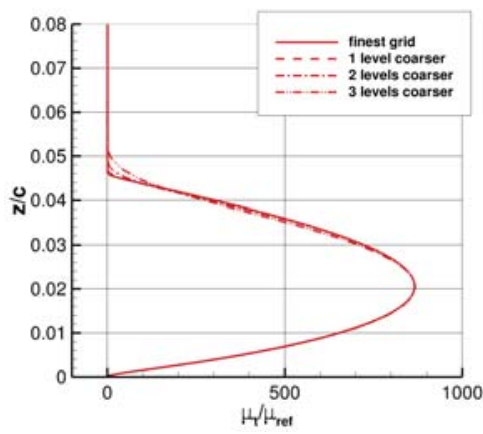

(b) FUN3D

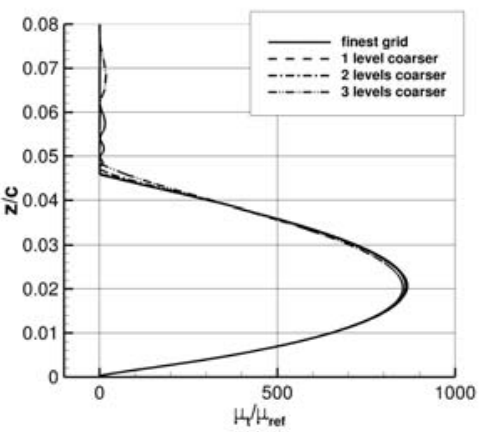

(c) TAU.

Figure 32. Eddy-viscosity variation along the line $x=0.999$ over the upper surface.

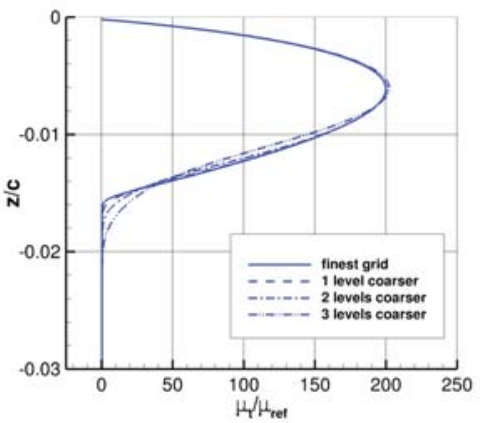

(a) CFL3D.

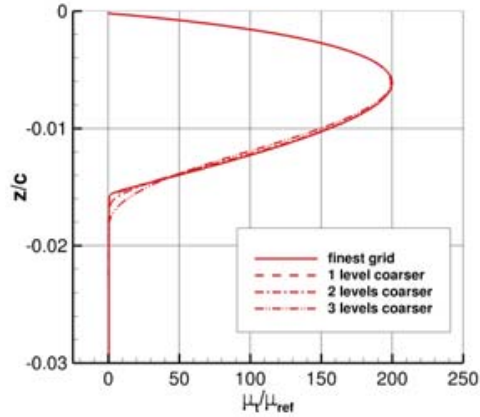

(b) FUN3D.

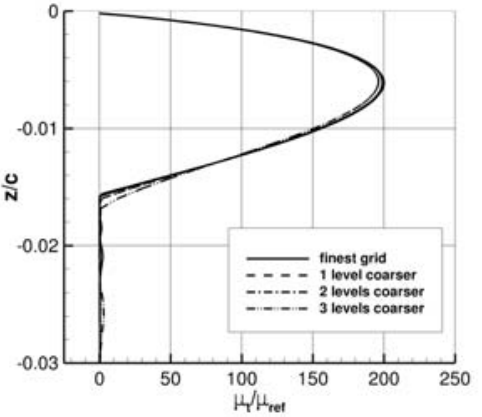

(c) TAU.

Figure 33. Eddy-viscosity variation along the line $x=0.999$ under the lower surface. 


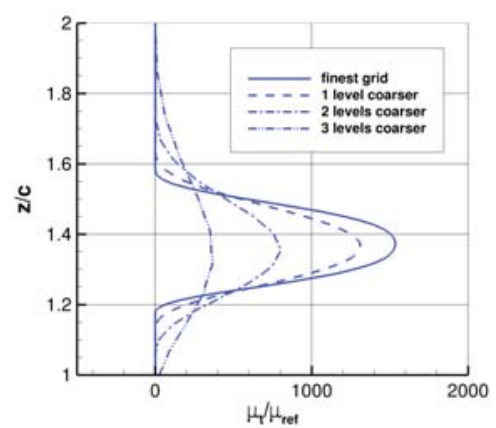

(a) CFL3D.

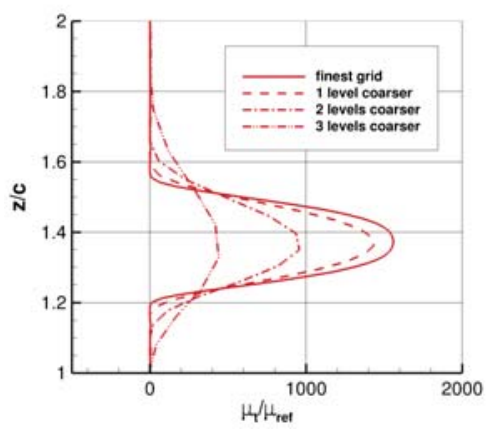

(b) FUN3D.

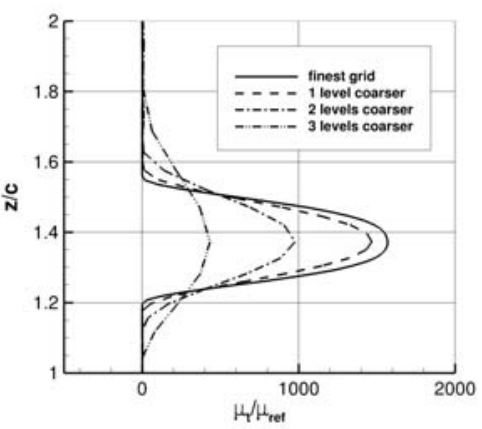

(c) TAU.

Figure 34. Eddy-viscosity variation in the wake along the line $x=10$.

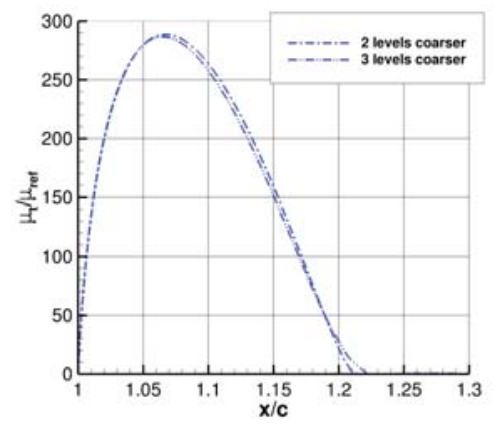

(a) CFL3D.

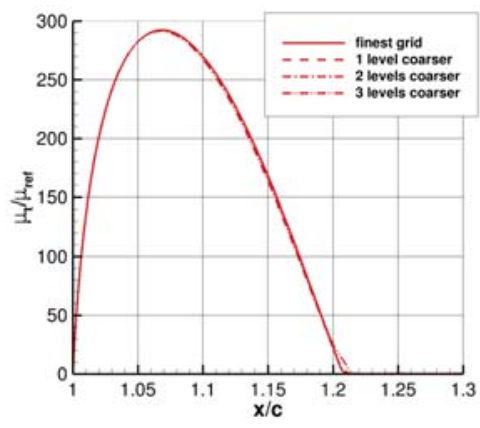

(b) FUN3D.

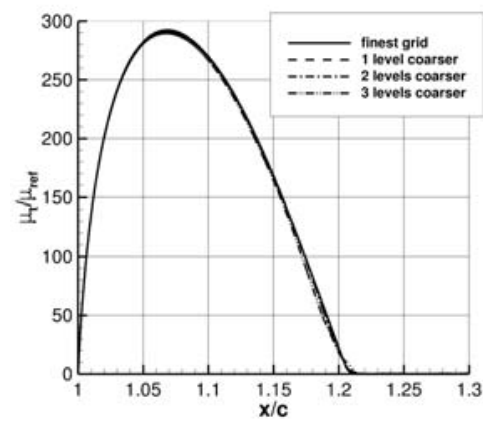

(c) TAU.

Figure 35. Eddy-viscosity variation behind the trailing edge along the line $z=0$.

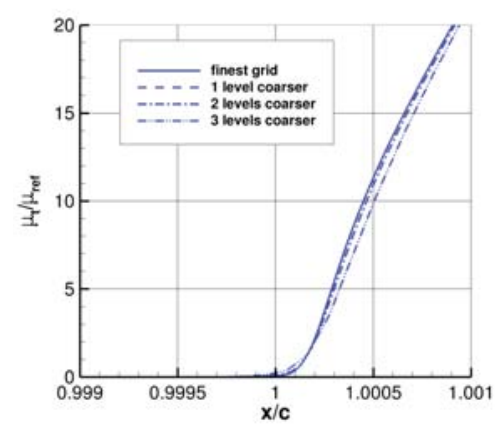

(a) CFL3D.

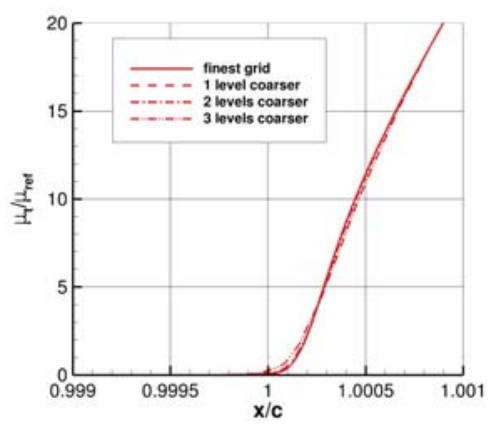

(b) FUN3D.

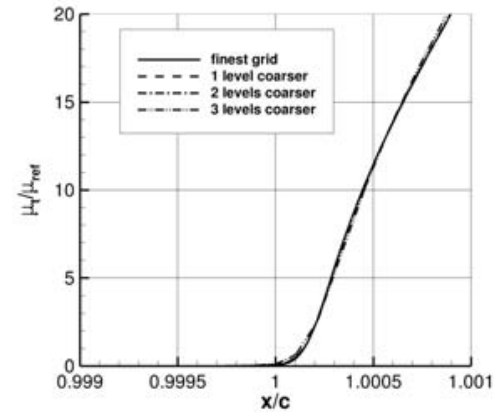

(c) TAU.

Figure 36. Eddy-viscosity variation near the trailing edge along the line $z=0.00008$. 


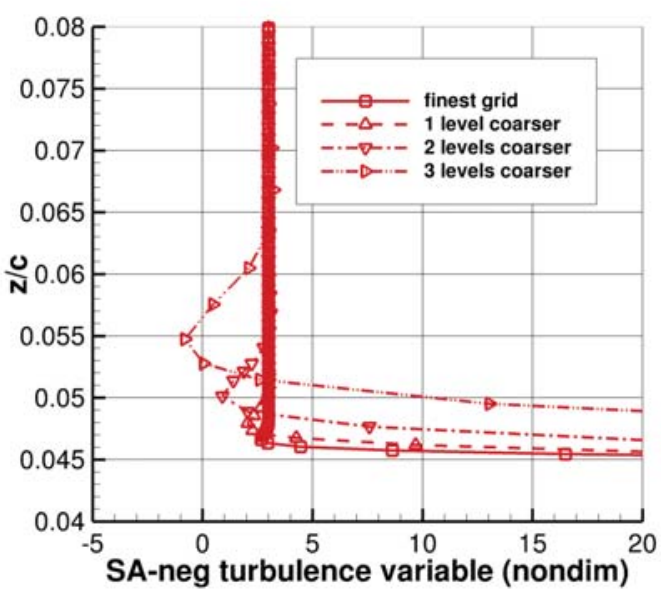

(a) $x=0.999$, upper surface, zoom to boundary layer edge.

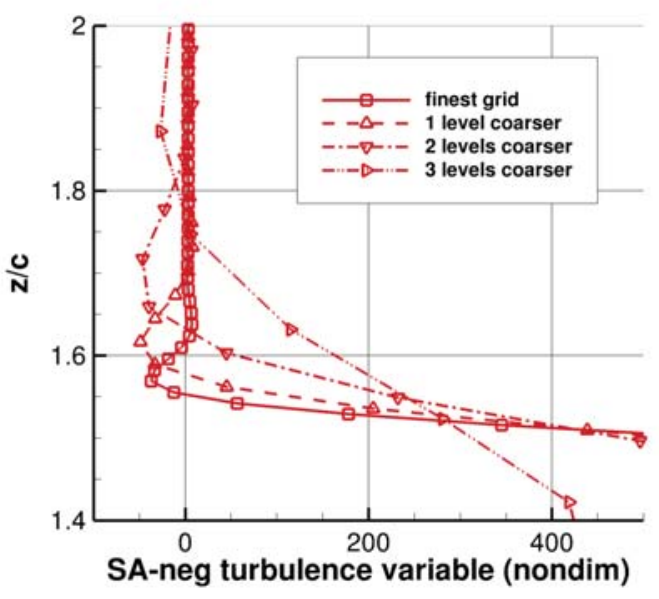

(b) $x=10$, zoom to wake edge.

Figure 37. FUN3D: Variation of the non-dimensional Spalart turbulence variable.

\section{F. Effects of Discretization Methods and Grid Elements}

Effects of variations in the FUN3D discretization methods and grid elements are studied for grids of Family I and Family II. The discretization methods vary in the approximation order of the convection term in the turbulence-model equation and the reconstruction method for the inviscid terms in the meanflow equations.

The baseline results correspond to a nominally second-order approximation of meanflow and turbulence-model equations. The meanflow variables are reconstructed from a node to the edge midpoints using the $\kappa=0.5$ MUSCL scheme. In the direction approximately tangential to the airfoil surface and horizontal in the wake, least-squares (lsq) gradients are used for reconstruction. The lsq gradients at a node are constructed from an unweighted least-squares linear fit to the primitive variables at the neighboring nodes. In the direction approximately normal to the airfoil surface and vertical in the wake, the gradients are computed using an implicit mapping along the structured grid lines approximately following this direction. Such gradients are denoted as mapped-n gradients. The second-order accurate discretization of the SA model convection term always uses lsq gradients for reconstruction of the Spalart turbulence variable from the node to the edge midpoint. The reconstruction corresponds to the $\kappa=0$ scheme. The baseline results on quadrilateral grids of Family II are expected to be the most accurate of the results presented.

Figures 38-40 compare convergence of the lift, pitching moment, viscous drag, and pressure drag coefficients. Each plot is characterized by four parameters: grid Family (I or II), grid element type (Q for quadrilateral elements or T for triangular elements), convection approximation order in the SA equation (1st or 2nd), and the type of gradients used for reconstruction in the normal direction (mapped-n or lsq). For example, the baseline discretization plot is designated as I:Q:2nd:mapped-n. Figure 38 shows convergence plots for different discretization methods on quadrilateral grids. The grid element designation $(\mathrm{Q})$ is omitted as it would be the same for all plots on the figure. The lift and pitching moment computed on grids of the same family with different discretization methods differ little in comparison to the differences between solutions on grids of different families. Note that errors introduced by poor trailing-edge resolution (on Family I grids) negate effects of more accurate discretization methods. In particular, lift and pitching moment coefficients computed on Family I grids with mapped-n gradient reconstruction appear less accurate than the coefficients computed with 1sq gradient reconstruction. The viscous drag coefficient computed from solutions with the first-order approximation in the SA equation is significantly lower than the coefficient computed from a solution with a second-order approximation, independent of the grid family. In pressure drag, the variations due to decreasing the accuracy of the turbulence-model convection term and the meanflow reconstruction method are in opposite directions. Thus, the pressure drag coefficients computed from the presumably most and least accurate solutions are similar on the third-finest grid. In either viscous or pressure drag, the total variation across all solutions on the finest grid is approximately 0.1 count.

Convergence of the aerodynamic forces and moment on triangular grids of Family I and Family II is shown in Fig. 39 and Fig. 40, respectively. The results on quadrilateral grids of Family I and II are shown for reference. 


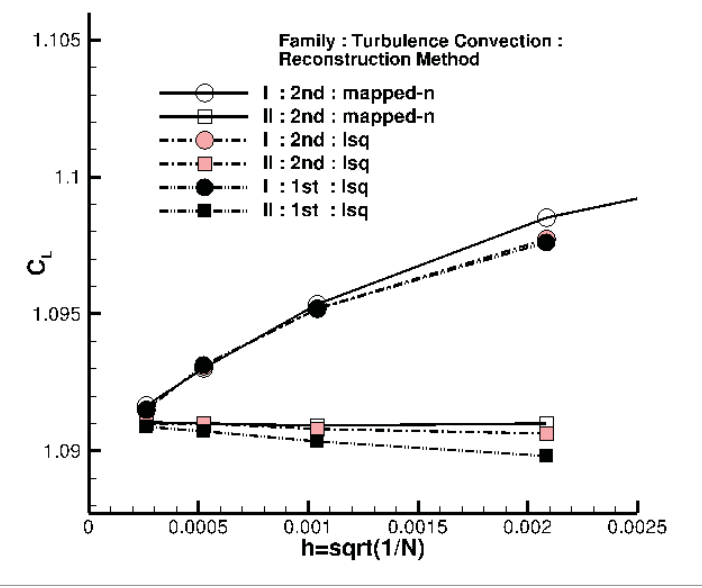

(a) Lift.

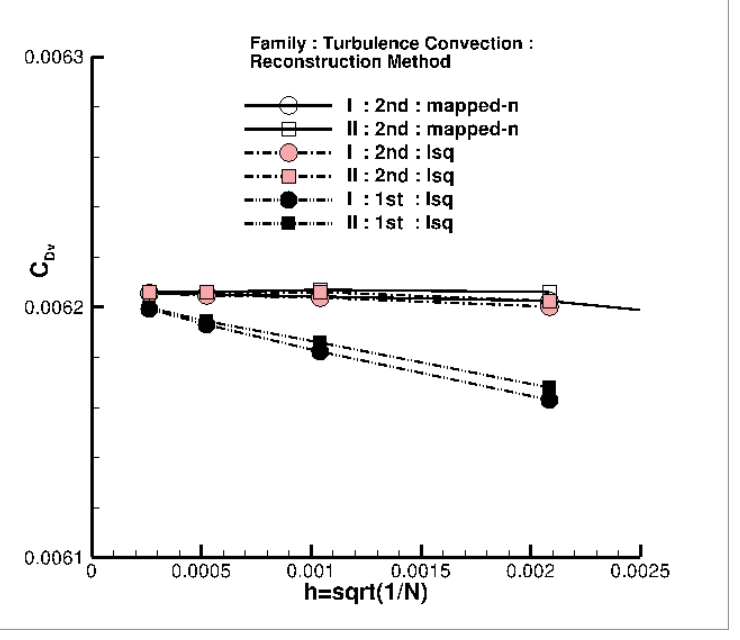

(c) Viscous drag.

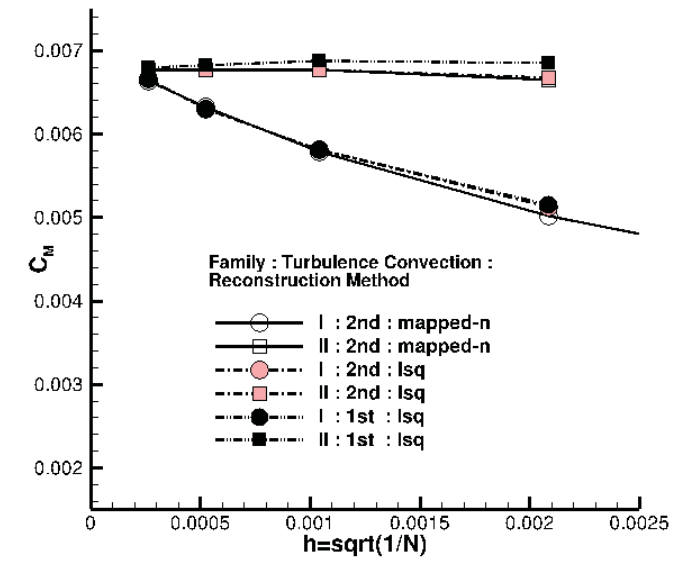

(b) Moment.

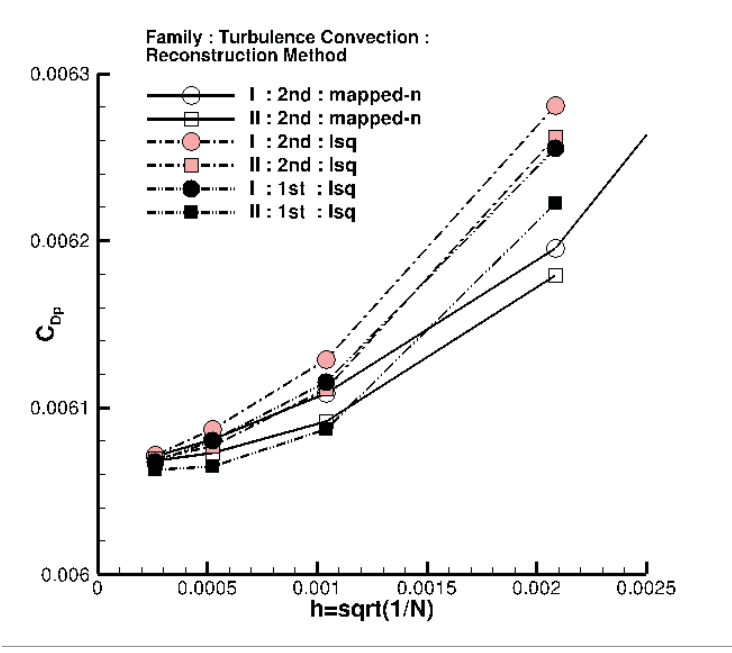

(d) Pressure drag.

Figure 38. Effect of variations in discretization method for quadrilateral grids. 
Approximation for the convection term in the SA model equation has a significant effect on solution accuracy on grids with triangular elements. The lift computed with the first-order approximation is reduced and the pitching moment is increased in comparison with the corresponding quantities computed using a second-order approximation. Solutions on triangular grids of Family I produce higher lift and pressure drag and a lower pitching moment than solutions on corresponding quadrilateral grids. Similar to computations on quadrilateral grids, the order of the convection-term approximation in the SA equation is the major factor affecting accuracy of the viscous drag coefficient. The viscous drag is significantly lower with the first-order approximation than with a second-order approximation independent of the grid elements, family, and meanflow flux reconstruction scheme. The sensitivities to triangular elements are more pronounced for Family I grids than for Family II grids. The solutions on triangular Family II grids computed with a second-order accurate SA-model convection term are very similar to the baseline solutions on quadrilateral Family II grids.

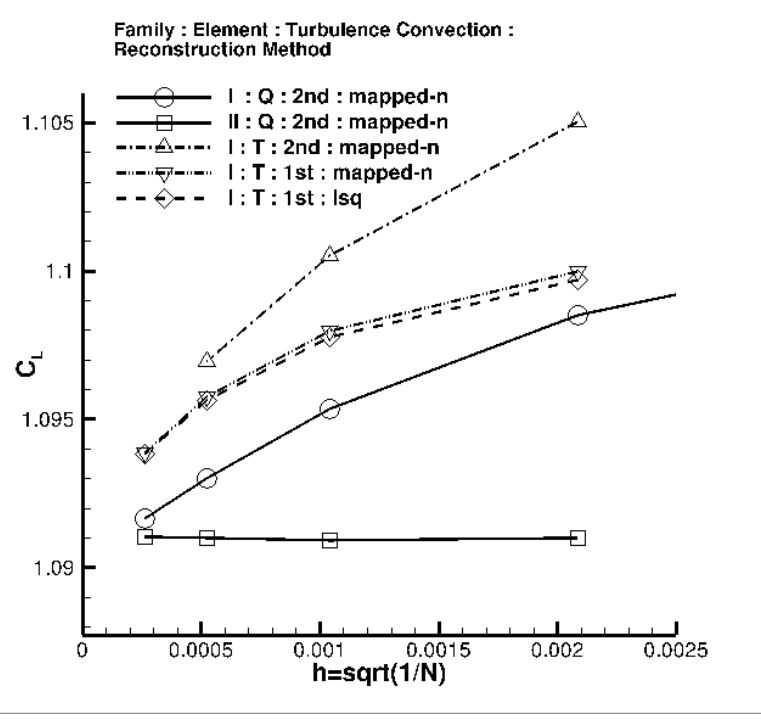

(a) Lift.

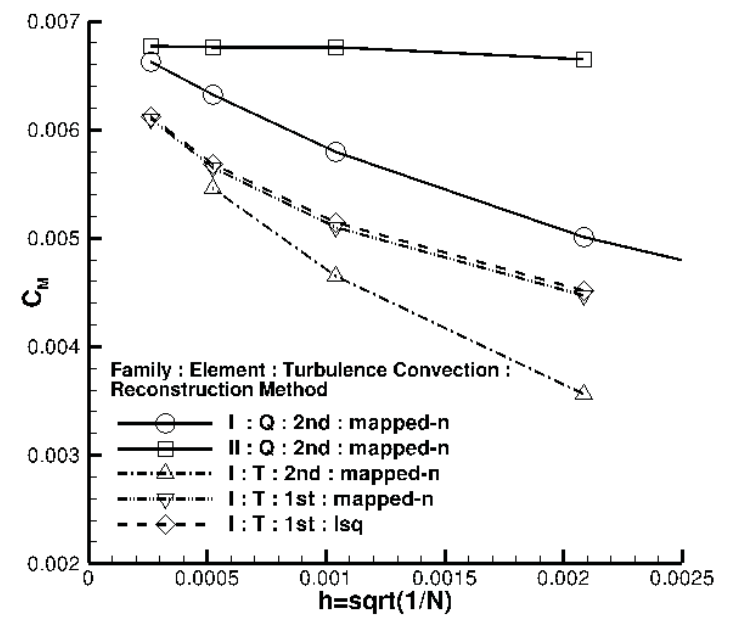

(b) Pitching moment.

(d) Pressure drag.

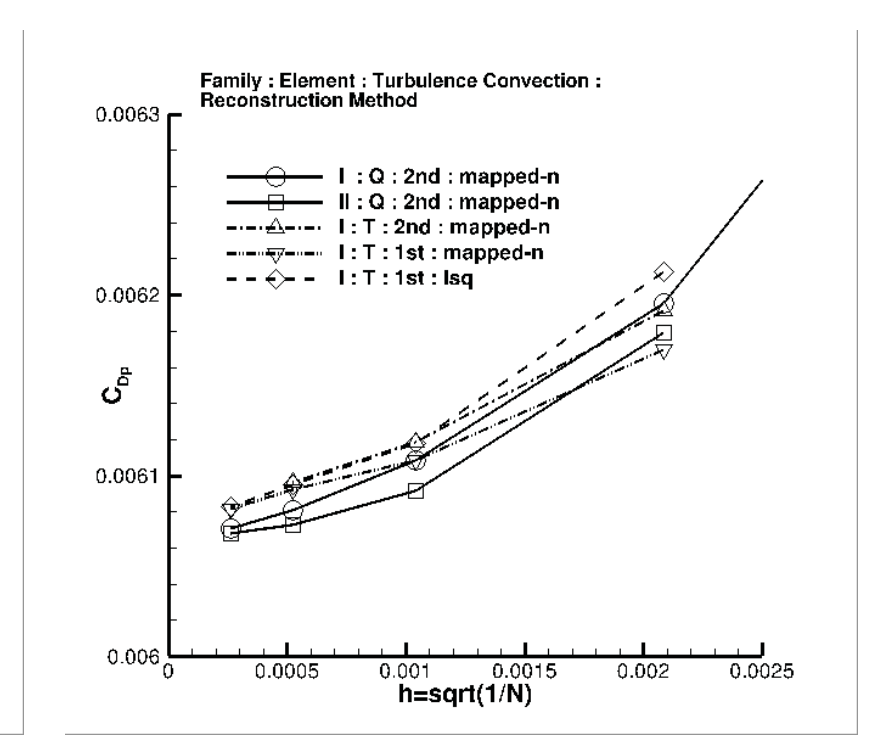

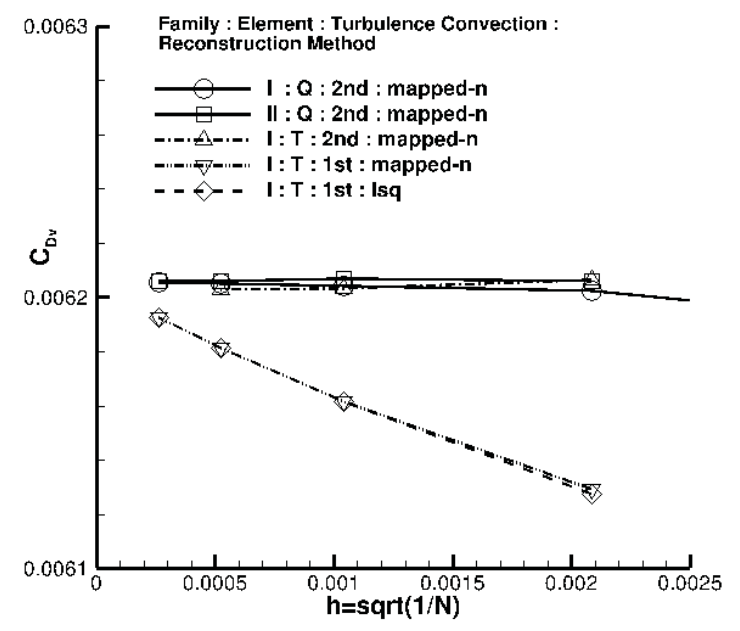

(c) Viscous drag.

Figure 39. Triangular grids of Family I: effect of discretization methods. 


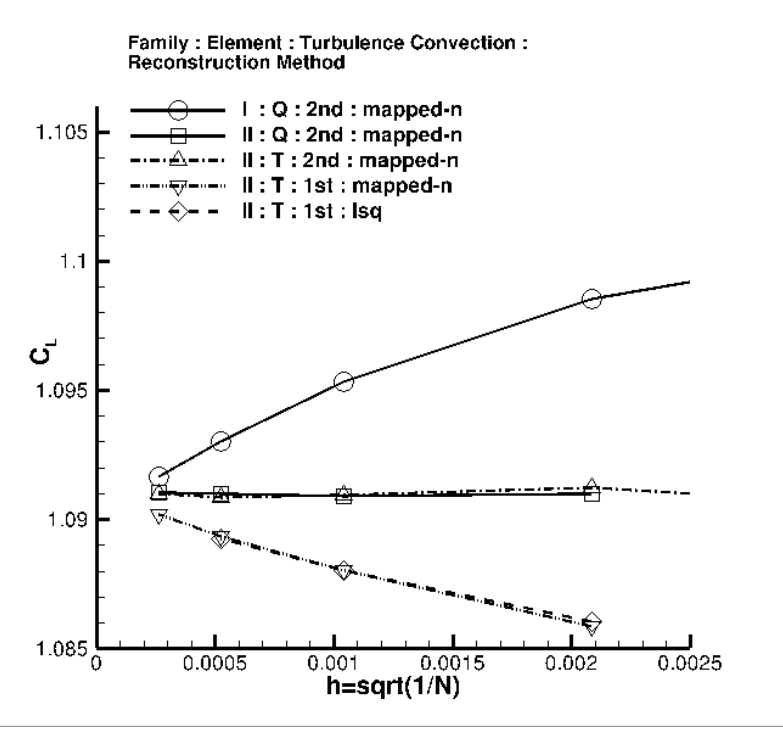

(a) Lift.

Family : Element : Turbulence Convection Reconstruction Method

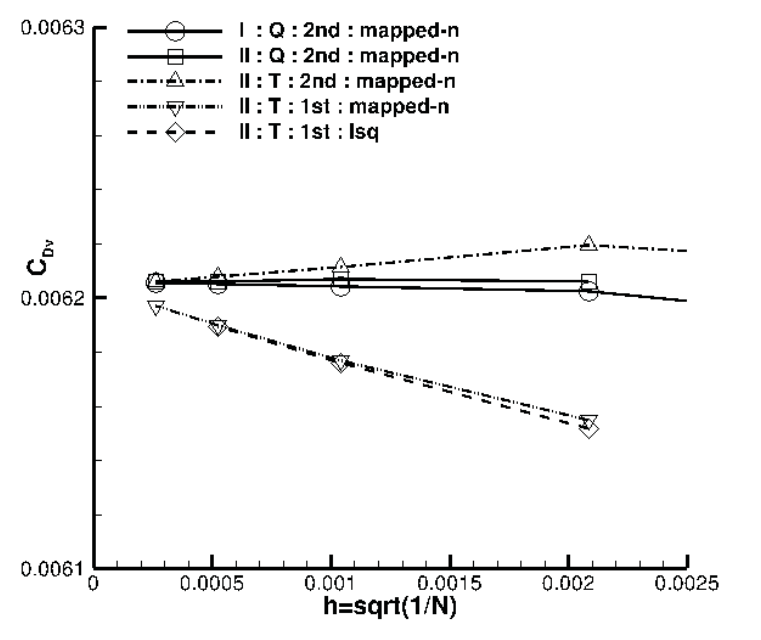

(c) Viscous drag.

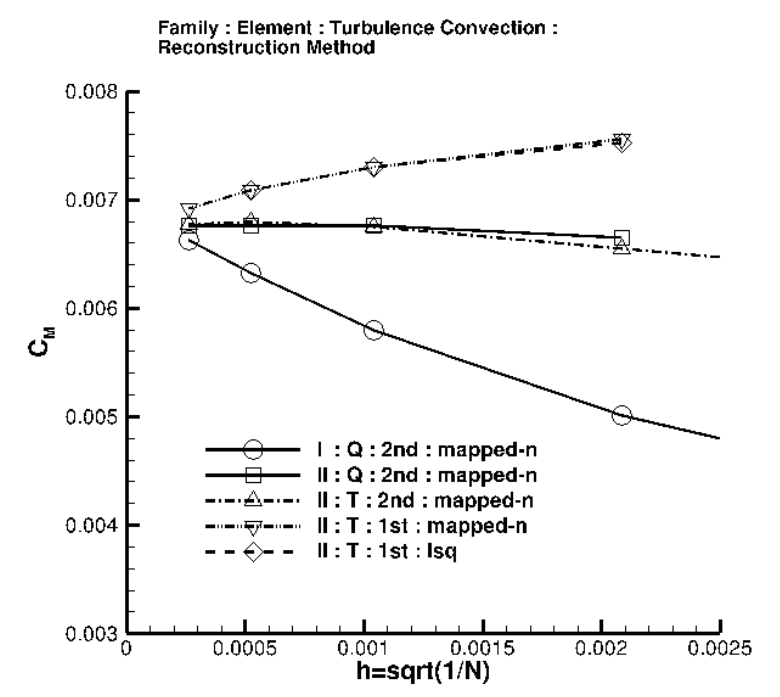

(b) Pitching moment.

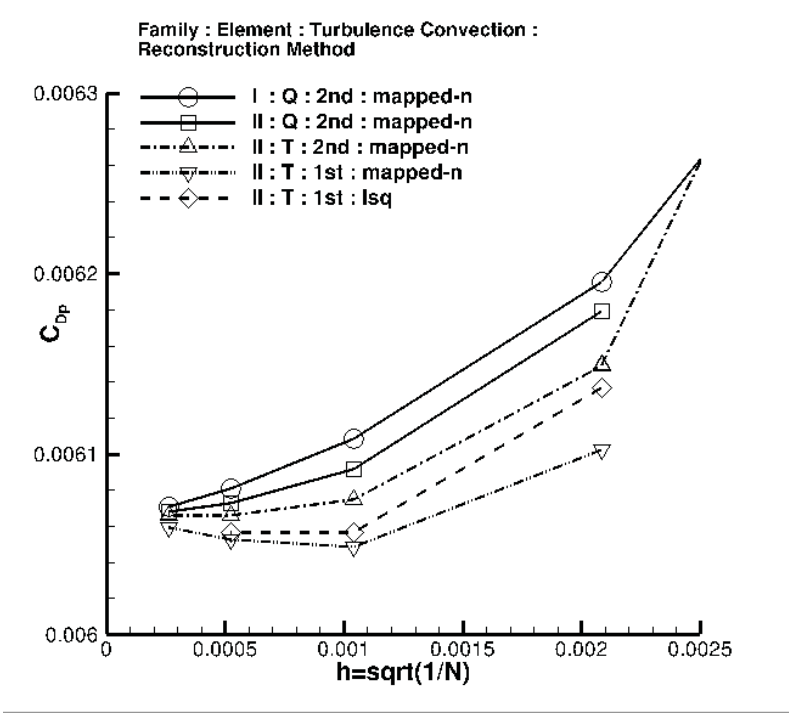

(d) Pressure drag.

Figure 40. Triangular grids of Family II: effect of discretization methods. 


\section{Flat Plate Configuration}

A grid convergence study for a turbulent flow through a cascade of finite flat plates is presented. FUN3D is used to establish an accurate reference solution and to assess effects of streamwise grid resolution near geometric singularities (i.e., the leading and trailing edges) on convergence of turbulent-flow solutions.

\section{A. Test Case Description}

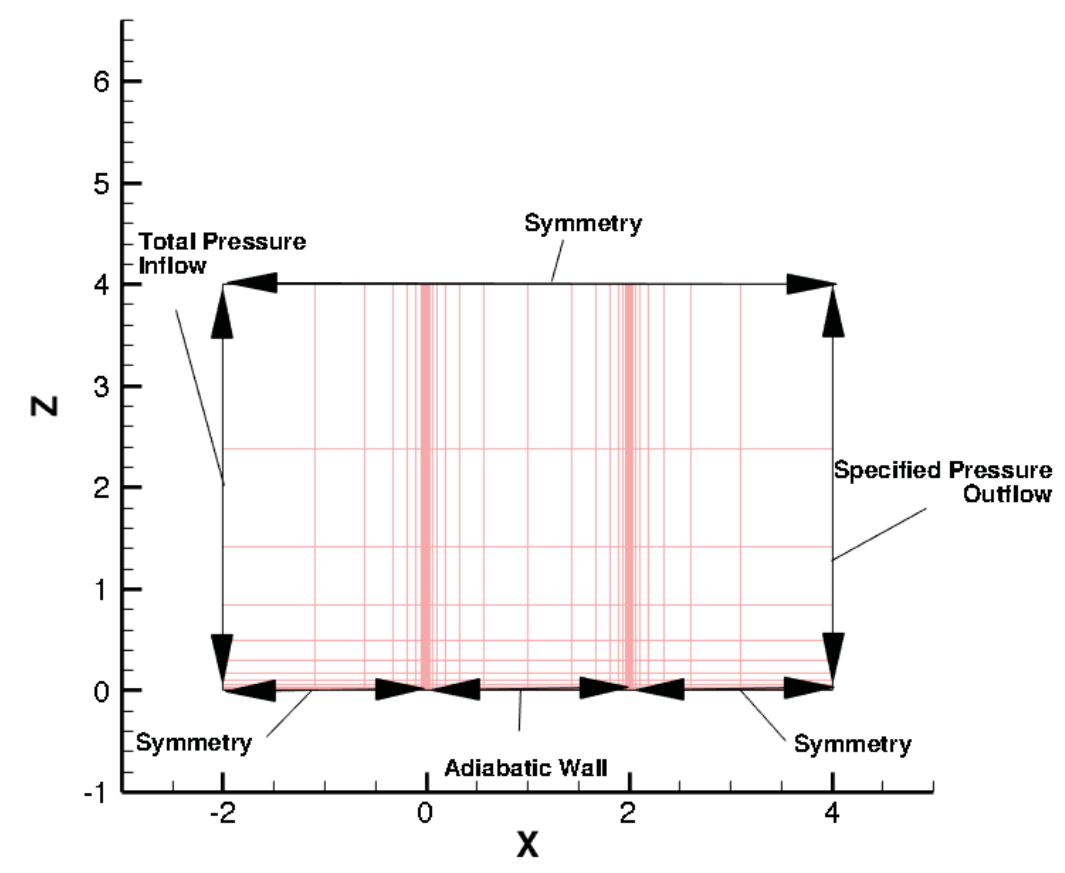

Figure 41. Boundary conditions for the finite flat plate geometry; $81 \times 25$ grid is shown.

This test case corresponds to the "2D Finite Flat Plate" case defined under the "Cases and Grids for Turbulence Model Numerical Analysis" section at the TMR website. The coordinate system is defined using non-dimensional units; $x$ and $z$ are streamwise and normal (vertical) coordinate directions, respectively. The boundary conditions are shown schematically in Fig. 41. The flat plate is located at the bottom of the domain $(0 \leq x \leq 2, z=0)$, similarly to the "2D Zero Pressure Gradient Flat Plate" case defined under the "Turbulence Model Verification Cases and Grids" section at the TMR website. For the current study, the computational domain $(-2 \leq x \leq 4,0 \leq$ $z \leq 4$ ) has been extended upward, upstream, and downstream of the plate, creating a trailing edge inside of the computational domain at $x_{T E}=2, z_{T E}=0$. The specific placement of the top, upstream, and downstream boundaries has been chosen to ensure that the drag coefficient is within 0.02 counts of the coefficient computed on a domain with such boundaries placed at infinity (see Sec. IV.F below). The top boundary condition has been changed to a symmetry condition to avoid specifying the external state along the top of the computational domain. Thus, the test case corresponds to a cascade of finite flat plates separated by a distance of eight in the vertical $z$-direction. Adiabatic no-slip boundary conditions are applied at the plate surface $(0 \leq x \leq 2, z=0)$. Constant total pressure boundary conditions, corresponding to $T_{t} / T_{r e f}=1+0.2 M_{\infty}^{2}$ and $P_{t} / P_{r e f}=\left(T_{t} / T_{r e f}\right)^{3.5}$, are applied at the upstream boundary $(x=-2,0 \leq z \leq 4)$. Constant pressure boundary conditions, corresponding to $P / P_{r e f}=1$, are applied at the downstream boundary $(x=4,0 \leq z \leq 4)$. Symmetry conditions are applied at the top $(-2 \leq x \leq 4, z=4)$ and bottom $(-2 \leq x \leq 0$ and $2 \leq x \leq 4, z=0)$ boundaries.

FUN3D computations have been performed on a series of nested, stretched, rectilinear grids ranging from the $2,561 \times 769$ (finest) grid to the $21 \times 7$ (coarsest) grid. The numbers in the grid notation indicate the points in the streamwise and normal directions, respectively. Each coarser grid is exactly every-other-line of the next finer grid. 
The grids are stretched in the wall-normal direction and are clustered near the leading and trailing edges. The normal spacing of the finest grid at the wall is $2.5 \times 10^{-7}$, corresponding to a non-dimensional boundary-layer spacing of $z^{+}=0.1$ at the middle of the plate $(x \approx 1)$. The $x$-directional clustering near the leading edge is set by specifying a local aspect ratio $\left(A R_{L E}\right)$ of the grid. The grids are symmetric about $x=1$ and thereby the trailing-edge aspect ratio is the same as at the leading edge. The recommended value, $A R_{L E}=1$, is used by default, although some computations on grids with $A R_{L E}=1200$, which is more typical of current practice, are reported in Sections IV.B and IV.C below. Figure 41 shows a view of the $81 \times 25$ grid.

The flow conditions correspond to $M_{\infty}=0.2$ and $R e=5 \mathrm{M}$ based on a unit length of the grid. The body reference length is two units. Thus, $R e=5 \mathrm{M}$ at the middle of the plate at $x=1$ and $R e=10 \mathrm{M}$ at the trailing edge of the plate at $x=2$. The SA-neg version of the Spalart-Allmaras turbulence model variable is used, although at convergence there are no negative values of the turbulence variable. The farfield value of the Spalart turbulence variable is $\tilde{\nu}_{\text {farfield }}=3 \nu_{\infty}$. The Prandtl number is taken to be constant at $\operatorname{Pr}=0.72$, and the turbulent Prandtl number is taken to be constant at $\operatorname{Pr}_{t}=0.9$. The molecular viscosity is computed using Sutherland's Law.

\section{B. Drag and Maximum Eddy Viscosity}

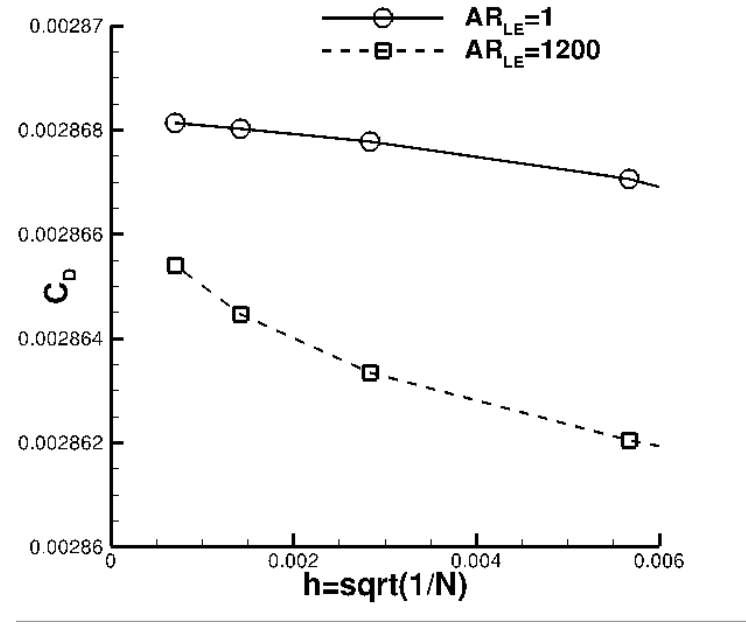

(a) Drag coefficient.

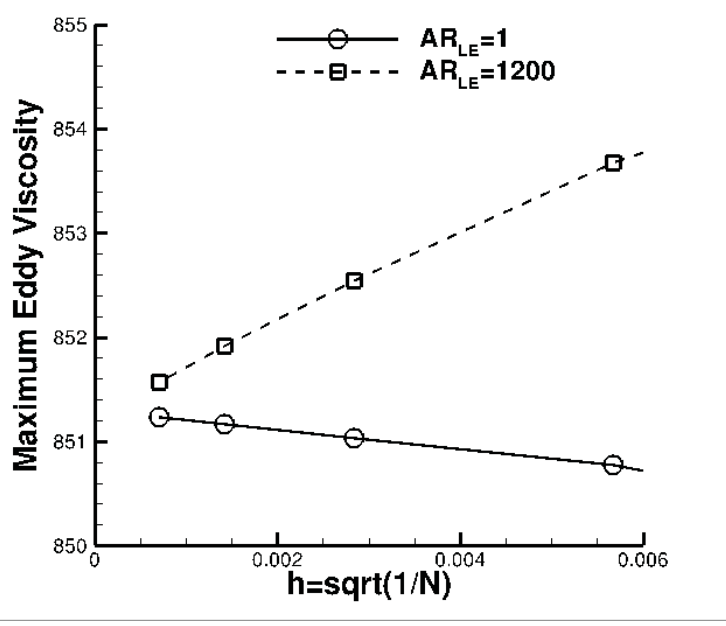

(b) Maximum eddy viscosity.

Figure 42. Grid convergence of drag and maximum eddy viscosity.

The convergence plots of the drag coefficient and the maximum eddy viscosity are shown in Fig. 42 for $A R_{L E}=1$ and $A R_{L E}=1200$ versus $h=\sqrt{1 / N}$. The drag scale is quite fine, spanning only 0.1 drag count. Both the drag coefficient and the maximum eddy viscosity show less variation in grid convergence with $A R_{L E}=1$ than with $A R_{L E}=1200$. The maximum eddy viscosity converges linearly in $h$ for each aspect ratio, indicating firstorder convergence. Close examination shows that the maximum eddy viscosity occurs just above the wake centerline (see Sec. IV.D below). The first-order variation of maximum eddy viscosity is believed to be a boundary effect, as grid convergence at a fixed location upstream of the trailing edge is approximately second order. In contrast, drag convergence is first order on the three finest grids for $A R_{L E}=1$ and less than first order for $A R_{L E}=1200$. From the boundary layer theory and the numerical results here, the skin friction in the leading edge varies as $O(1 / \sqrt{x})$. The drag integration routine is a trapezoidal second-order integration, so drag convergence is expected to be first order even if the skin friction values were exact.

To investigate convergence of local drag contributions, three sections on the surface are chosen: near the leading edge, in the middle of the plate, and near the trailing edge; the sections are defined in Table 3 . The separator nodes, $(x, z)=(0.107267441655523,0)$ and $(x, z)=(1.89273255834448,0)$, are present on the finest four $A R_{L E}=1$ grids used in the investigation. Convergence of drag within each of the sections is shown versus $h^{p}$ in Fig. 43 for various choices of $p$. The dashed lines in the figure are linear fits for the finest two grids. In the leading-edge section, the drag convergence order is slightly less than first, as would be expected. In the middle-plate section, the drag 


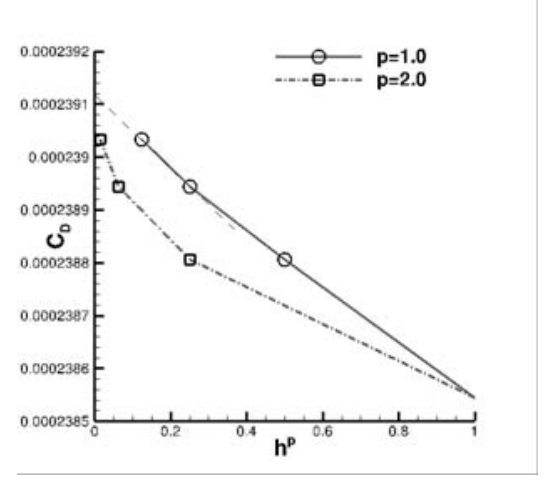

(a) Leading-edge section.

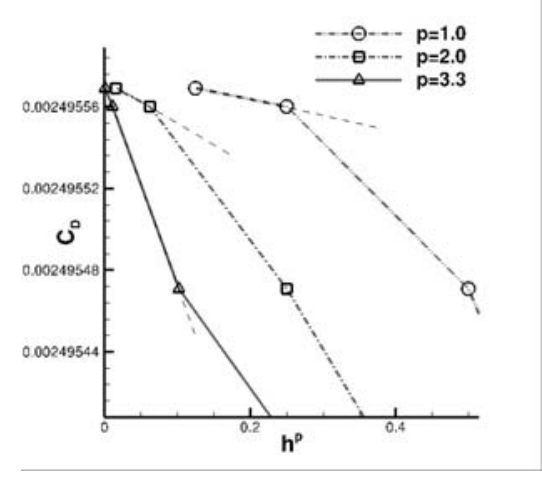

(b) Middle section.

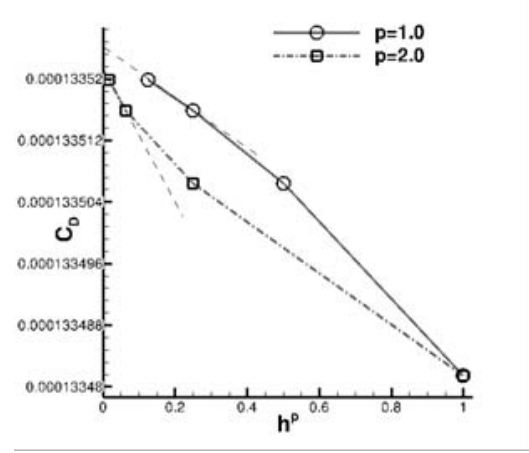

(c) Trailing-edge section.

Figure 43. Grid convergence of drag within different sections.

convergence order is high, close to $p=3.3$, which is unexpected. In the trailing-edge section, the drag convergence order is slightly greater than first.

Table 3. Sections of the plate.

Leading Edge

Middle Plate

Trailing Edge

$0 \leq x \leq 0.107267441655523 \quad 0.107267441655523 \leq x \leq 1.89273255834448 \quad 1.89273255834448 \leq x \leq 2$

\section{Skin Friction, Surface Pressure, and Boundary-Layer Profile}

The skin friction variation near the leading edge is shown in a global view (Fig. 44 (a)) and in a local view near $x=0.000101$ (Fig. 44 (b)). Four finest grids with $A R_{L E}=1$ are used: "grid 1" designates the finest $2,561 \times 769$ grid, "grid 4" designates the $321 \times 97$ grid. An approximate analytic fit to the skin friction variation corresponding to $0.0002503 x^{-0.52}$ is shown, with the constant selected to match the infinite-grid extrapolated value at $x=0.000101$ assuming second-order grid convergence. In Fig. 44 (a), the maximum discretization error actually grows on finer grids. The maximum relative discretization error, defined as the local discretization error divided by the local solution value, reduces quite slowly in grid refinement. In Fig. 44 (b), the skin friction plots on the two finest grids are indistinguishable. The skin friction at $x=0.000101$ is shown in Fig. 45 versus $h$ and $h^{p}$ to assess grid convergence. The dashed lines in the figures fit the results on the two finest grids. Grid convergence exhibits an order very close to 1.6 on the three finest grids.

Skin-friction convergence in the middle of the plate at $x=1.0$ is shown in Fig. 46 for $A R_{L E}=1$ and $A R_{L E}=$ 1200. The $A R_{L E}=1$ results show an apparent convergence order of 2.5; the $A R_{L E}=1200$ results show less-thanfirst convergence order. Somewhat surprisingly, the $A R_{L E}=1200$ results have smaller discretization error levels on coarser grids; the errors are extremely small for all solutions.

The skin friction variation near the trailing-edge region is shown in a global view (Fig. 47 (a)) and near $x=1.95$ (Fig. 47 (b)). Results with both $A R_{L E}=1$ and $A R_{L E}=1200$ are shown. The authors could not find a simple function to characterize the variation of the skin friction in this range; the function $0.0027001033 x^{-0.14}+0.000007 /(2-x)^{0.5}$ is shown to indicate that the skin friction is singular near the trailing edge. Assuming that the $A R_{L E}=1$ solution on grid 1 is reasonably close to the exact solution, large errors in $A R_{L E}=1200$ solutions are evident over the four grid points closest to the trailing edge and the maximum $A R_{L E}=1200$ discretization error decays very slowly, if at all. The $A R_{L E}=1$ solutions also exhibit the largest errors at the four grid points closest to the trailing edge. Grid convergence at the trailing edge is not analyzed in detail, but can be inferred from Fig. 47 (b). The $A R_{L E}=1$ results exhibit low discretization errors and a better-than-first grid convergence order, while the $A R_{L E}=1200$ results show larger discretization errors and an apparent less-than-first grid convergence order.

Convergence of the pressure coefficient, $C_{p}$, near the leading and trailing edges is shown in Fig. 48. The pressure appears discontinuous at both the edges along the line tangential to the plate. Grid convergence is slow at the grid 


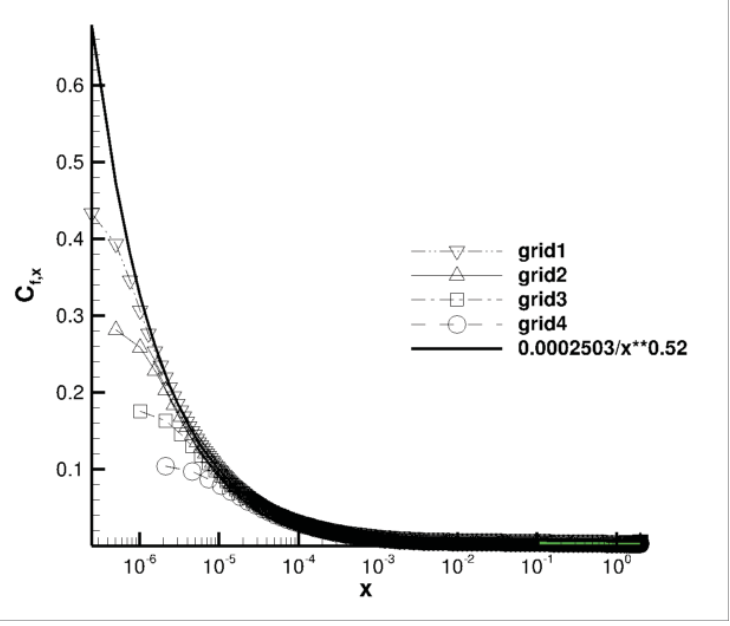

(a) Global view.

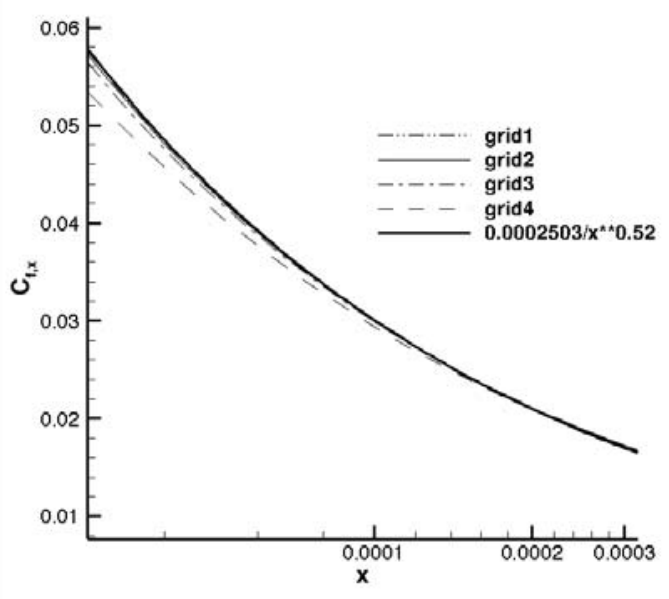

(b) Near $x=0.0001$.

Figure 44. Skin friction convergence in leading-edge region; $A R_{L E}=1$.
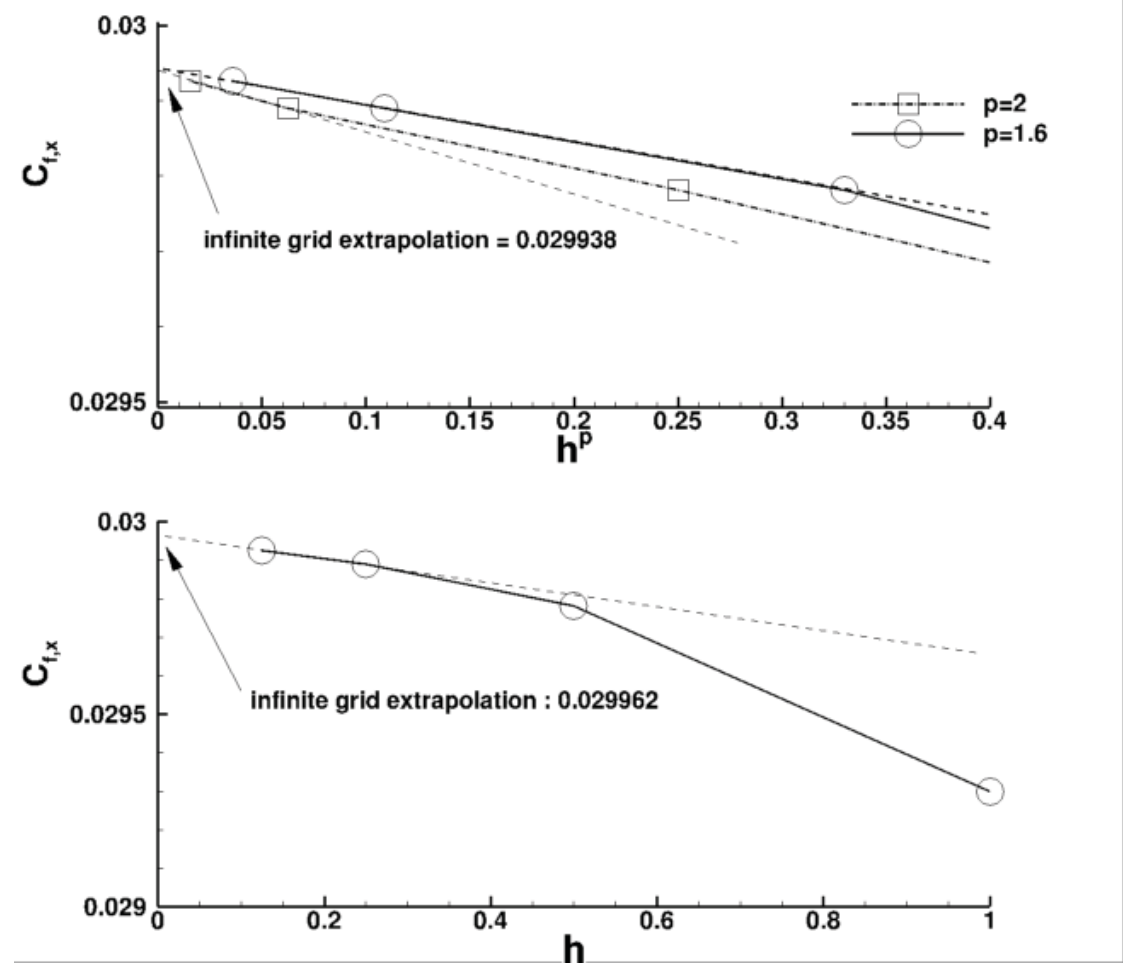

Figure 45. Skin friction grid convergence at $x=0.000101 ; A R_{L E}=1$ 

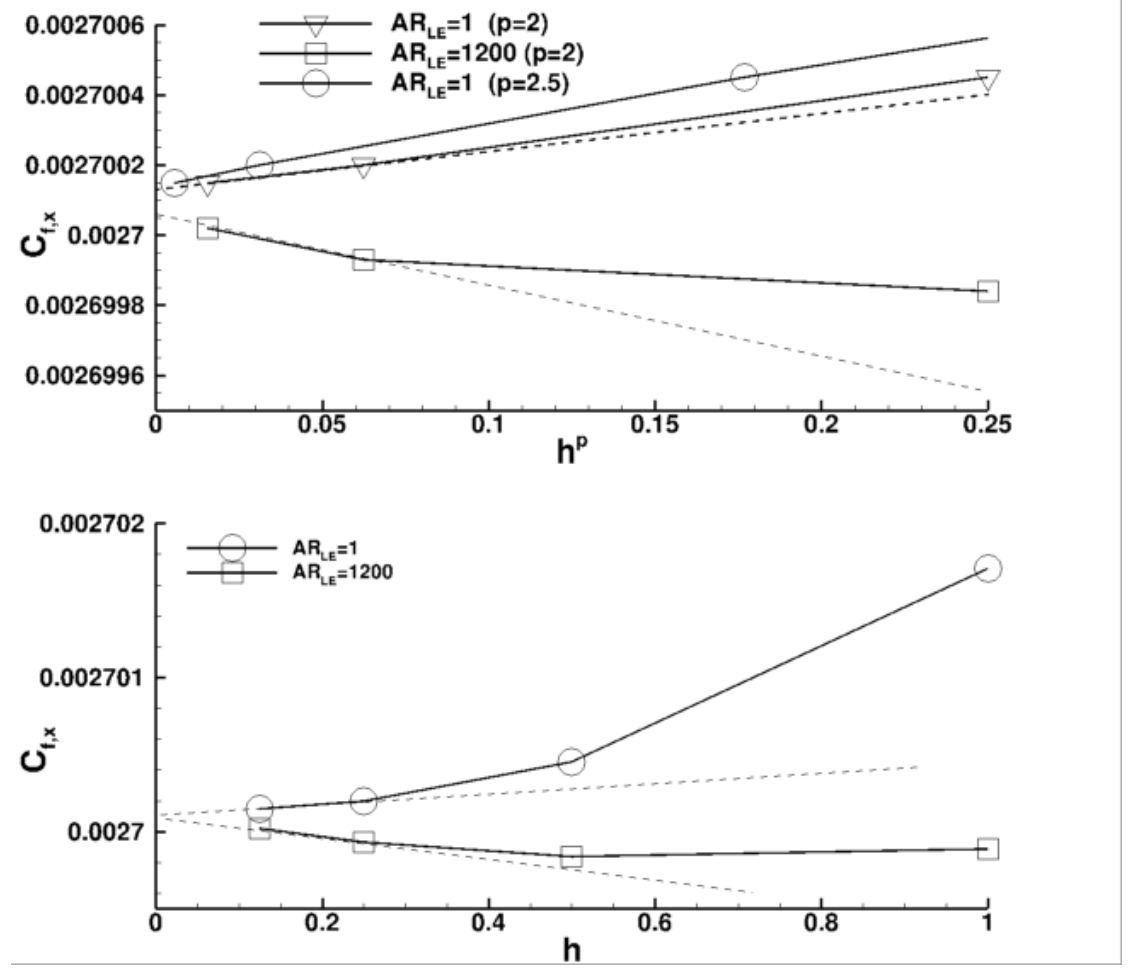

Figure 46. Skin friction grid convergence at $x=1.0$.

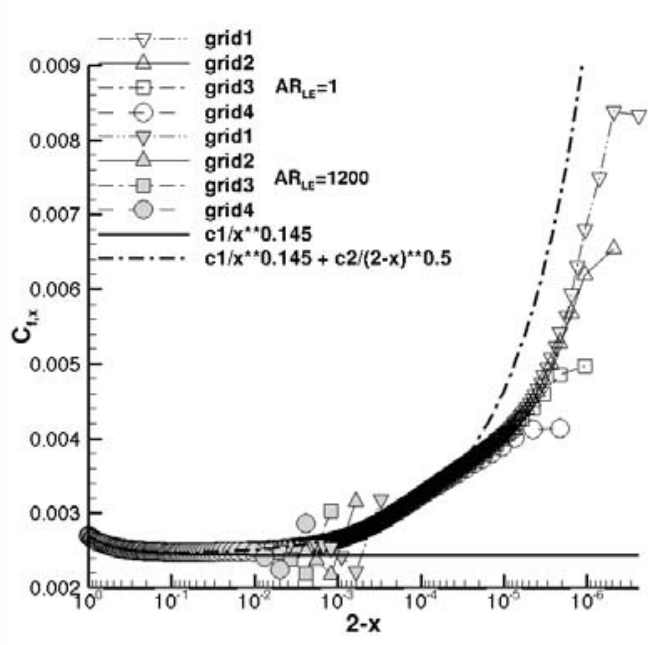

(a) Global view; $\mathrm{c} 1=0.0 .0027001033 ; \mathrm{c} 2=0.000007$.

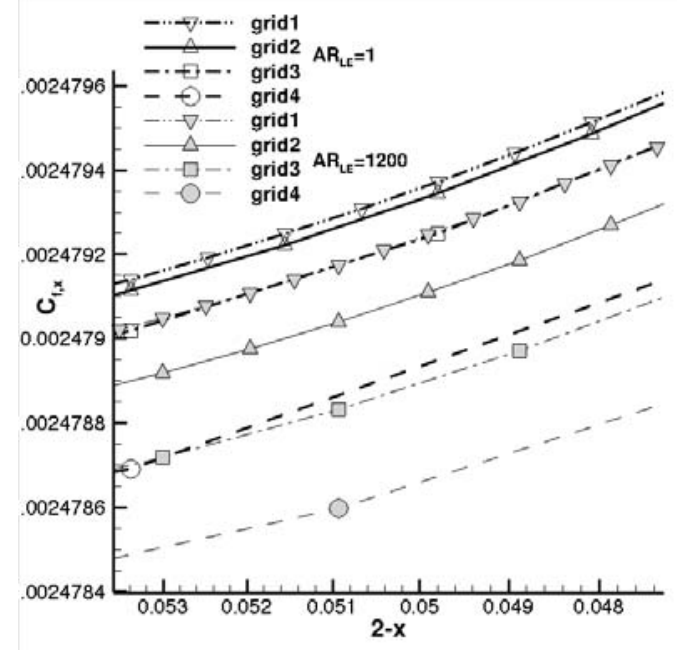

(b) Near $x=1.95$.

Figure 47. Skin friction convergence near trailing edge. 
points immediately adjacent to the edges but, at a fixed distance away from the edges, convergence is approximately second order. Contours of the pressure coefficient near the leading and trailing edges are shown in Figs. 49 (a) and (b), respectively. Near both the edges, the pressure coefficient varies smoothly along rays emanating from the edges.

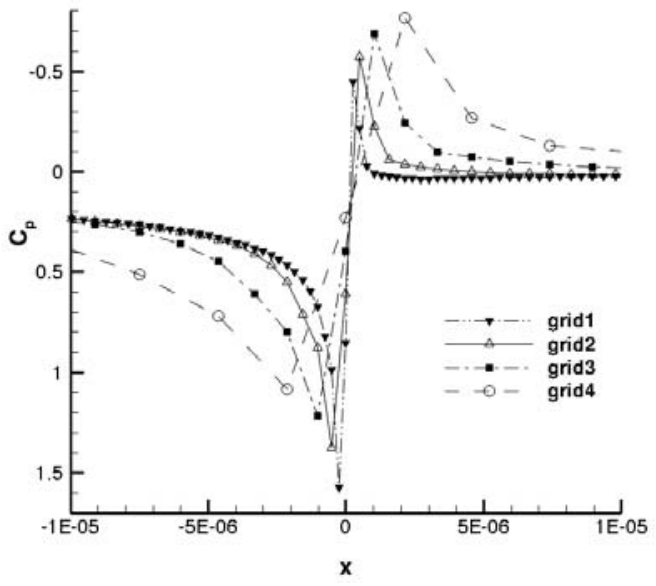

(a) Near leading edge.

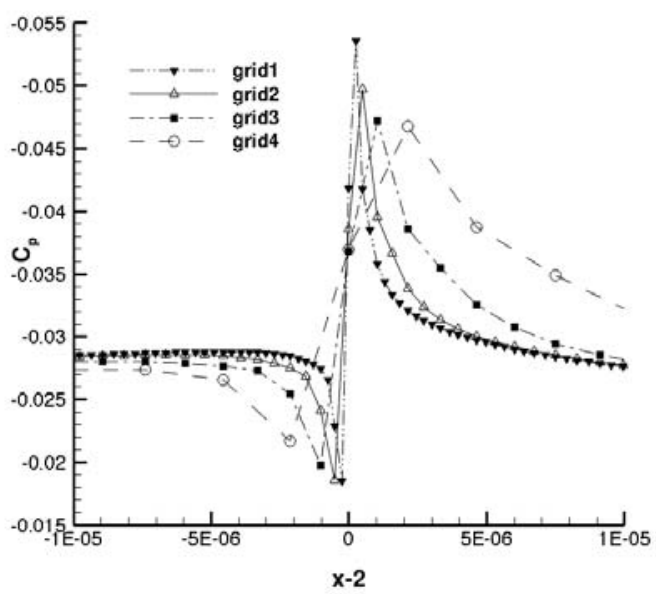

(b) Near trailing edge.

Figure 48. Surface pressure coefficient.

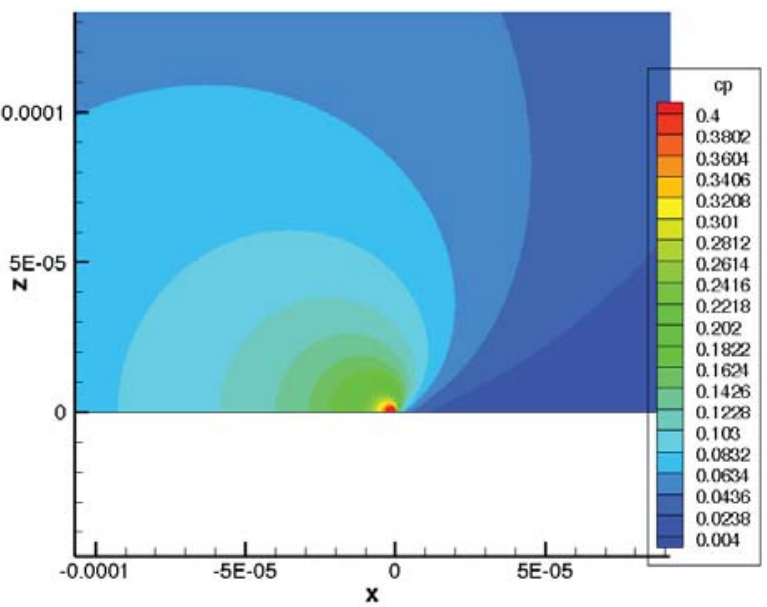

(a) Near leading edge.

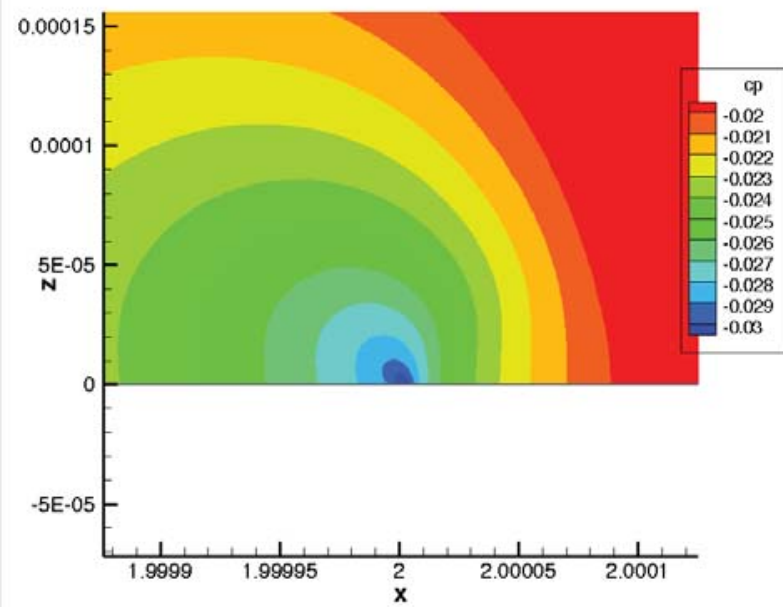

(b) Near trailing edge.

Figure 49. Contours of pressure coefficient.

Figure 50 shows the boundary layer profiles of the horizontal velocity, $u$, in the middle of the plate at $x=1$. In the global view with a logarithmic $z$-scale, the velocity profiles computed on different grids are indistinguishable from each other. In a detailed view near $z \approx 0.001$, grid convergence is qualitatively second order. Similarly, in the global view (Fig. 51 (a)), the eddy viscosity profiles are indistinguishable from each other; and the detailed view near the edge of the boundary layer (Fig. 51 (b)) reveals grid convergence that is qualitatively better than first order. 


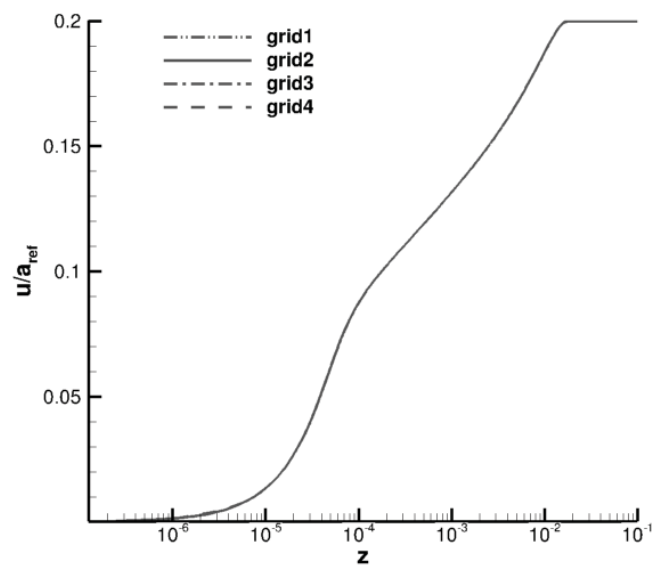

(a) Global view.

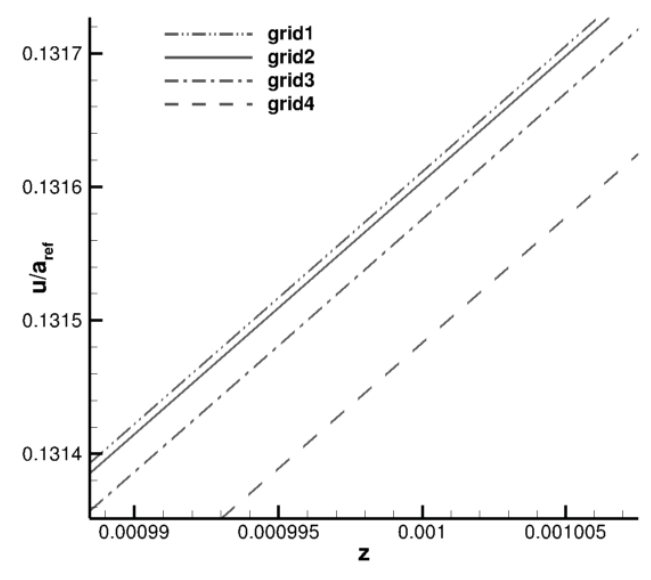

(b) Near middle of logarithmic region.

Figure 50. Velocity profile at $x=1$.

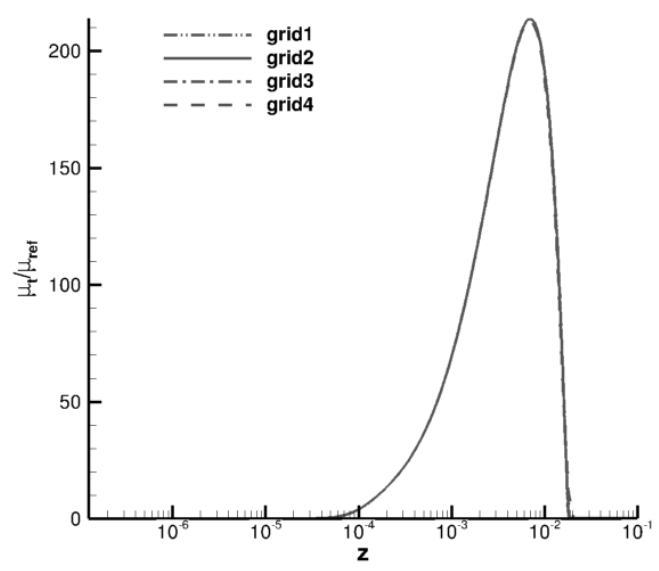

(a) Global view.

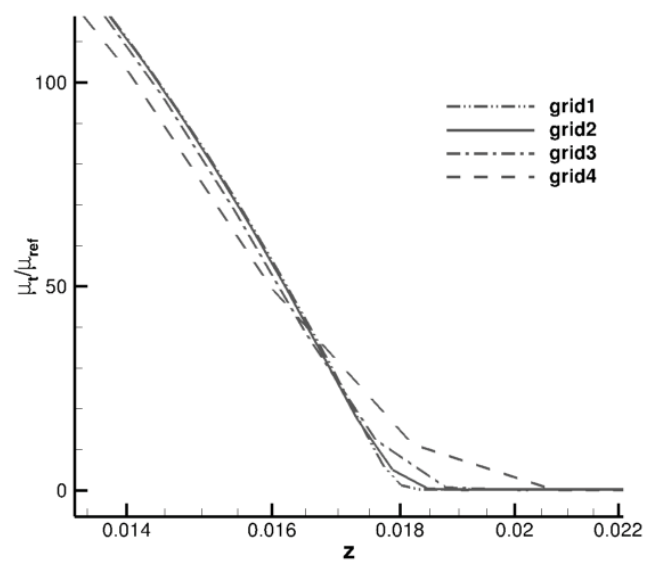

(b) Near edge of boundary layer.

Figure 51. Eddy viscosity profile at $x=1$. 


\section{Downstream of Trailing Edge}

Figures 52 and 53 show grid convergence of the horizontal velocity, $u$, downstream of the trailing edge. Away from the trailing edge, the horizontal velocity increases as $\left(x-x_{T E}\right)^{0.08}$ and the velocity distributions on different grids are nearly indistinguishable from each other in the global view (Fig. 52 (a)). Near the trailing edge (Fig. 52 (b)), the maximum errors are reducing slowly as the grid is refined. At a small distance from the trailing edge, the velocity variation slope is changed to $\left(x-x_{T E}\right)^{0.43}$, and the errors converge with the rate between first and second order. Figures 53 and 54 illustrate convergence at locations corresponding to close proximity to the trailing edge $\left(\left(x-x_{T E}\right) \approx\right.$ $\left.10^{-4}\right)$ and at a location further in the wake $(x \approx 3)$. At both locations the apparent convergence order is 1.6. Note that the turbulent-flow velocity profile near the trailing edge is similar to the profile of a reentrant-corner solution for a pure-diffusion equation described in the Appendix, but the observed convergence rate is higher. The pure-diffusion solution exhibits a square-root behavior near the singularity and first-order convergence at any fixed interior location.

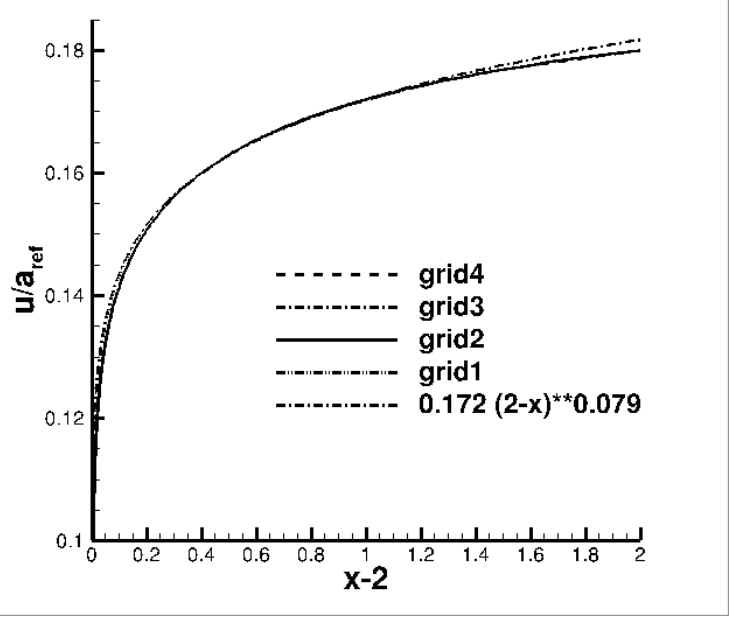

(a) Global view.

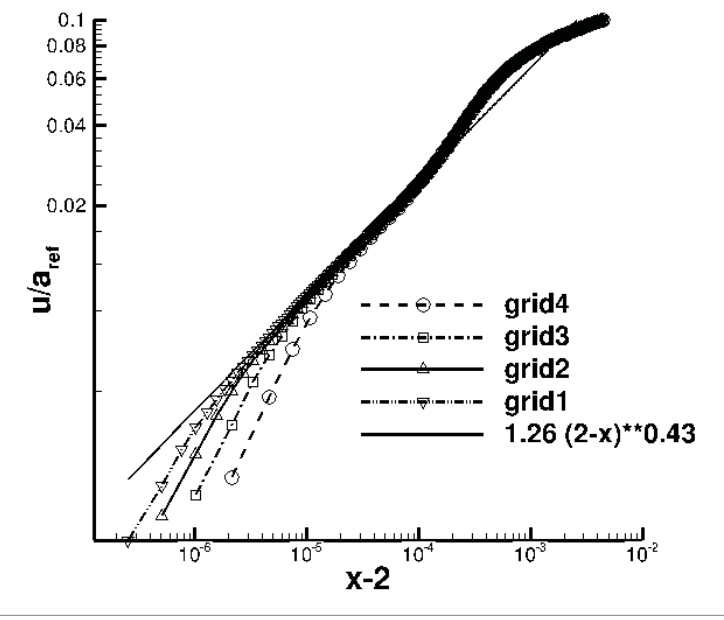

(b) Near trailing edge.

Figure 52. Velocity downstream of the trailing edge.

Figures 55 and 56 show grid convergence of the pressure $\left(C_{p}\right)$ and eddy viscosity $\left(\mu_{t}\right)$ downstream of the trailing edge. The pressure distributions (Fig. 55 (a)) on different grids are nearly indistinguishable from each other, except in an immediate vicinity of the outflow boundary, where the specified-pressure boundary condition forces sharp solution variations over a few grid points adjacent to the boundary. Fig. 55 (b) indicates that, in the trailing-edge vicinity, maximum errors in pressure are at the grid points nearest the edge. Note grid convergence in Fig. 55 (b) is the same as in Fig. 48 (b), but shown with a logarithmic scale for the abscissa. At a fixed distance away from the trailing edge, grid convergence is qualitatively second order.

The eddy viscosity distributions behind the trailing edge computed on different grids are nearly indistinguishable from each other (Fig. 56 (a)). Although not shown, examination of the eddy viscosity at a fixed distance from the trailing edge shows better-than-first-order grid convergence.

Figures 57 and 58 show the wake profiles of the horizontal velocity and eddy viscosity at $x=3$. In the global view, the velocity profiles computed on different grids are indistinguishable from each other except near the edge of the wake. In a detailed view near the edge of the wake, grid convergence is qualitatively second order. Similarly, the eddy viscosity profiles from the three finest grids are close to each other in the global view with small deviations near the edge of the wake; and the detailed view near the maximum-eddy-viscosity location reveals grid convergence that is qualitatively second order.

\section{E. Iterative Convergence}

Figures 59 (a) and (b) illustrate iterative convergence of the Full Multigrid (FMG) solver ${ }^{35}$ on grids with $A R_{L E}=$ 1200 and $A R_{L E}=1$, respectively. Convergence of the $L_{2}$ norms of the meanflow and turbulence-model residuals and the drag coefficient is shown. Only four grids are used in the FMG process. A Full Approximation Scheme (FAS) 


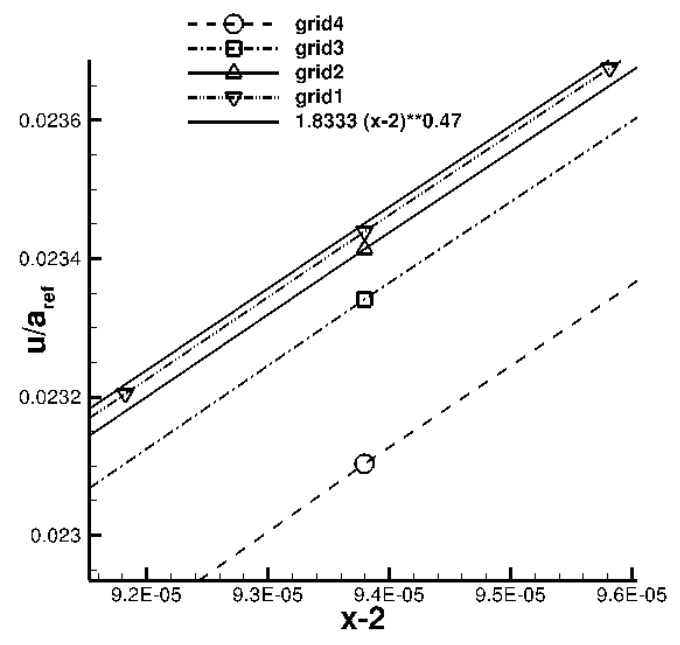

(a) Local view.

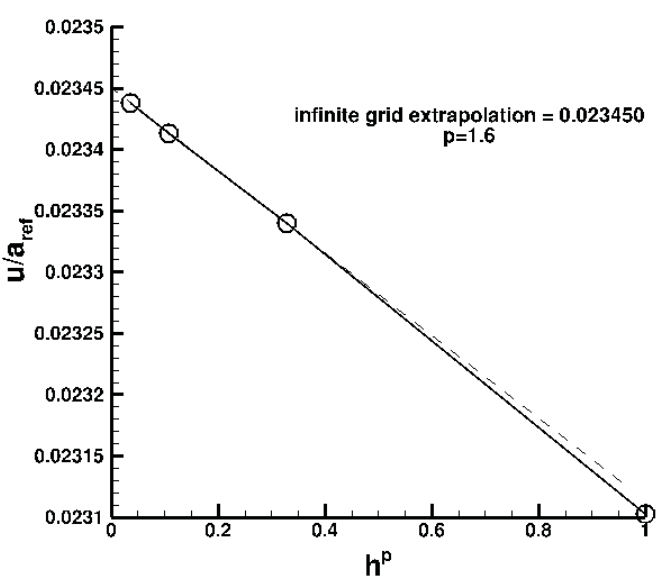

(b) Convergence order.

Figure 53. Velocity convergence at $x-x_{T E} \approx 10^{-4}$.

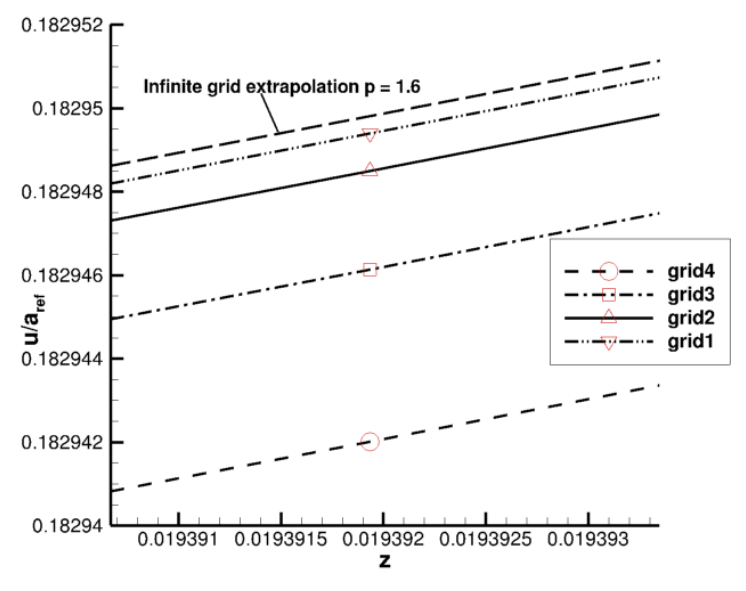

(a) Local view.

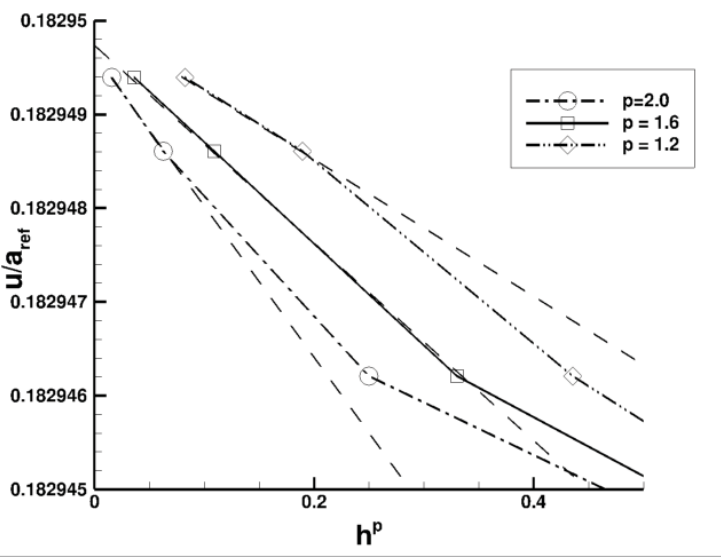

(b) Convergence order.

Figure 54 . Velocity convergence at $x \approx 3$. 


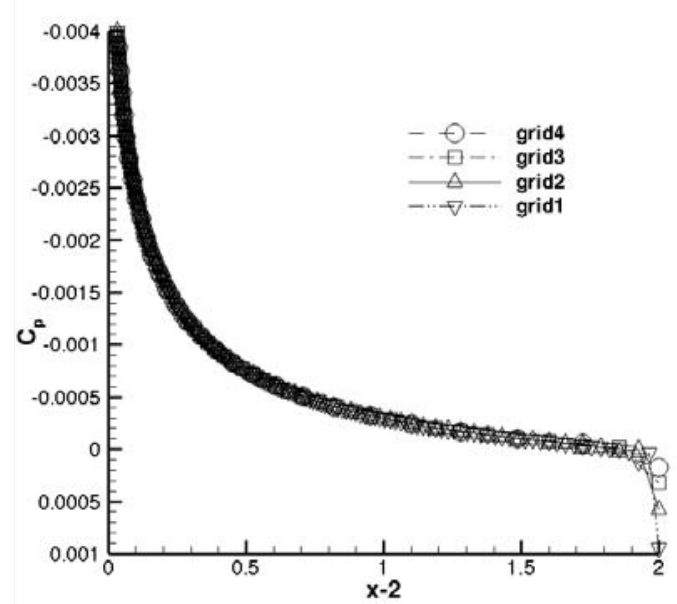

(a) Global view.

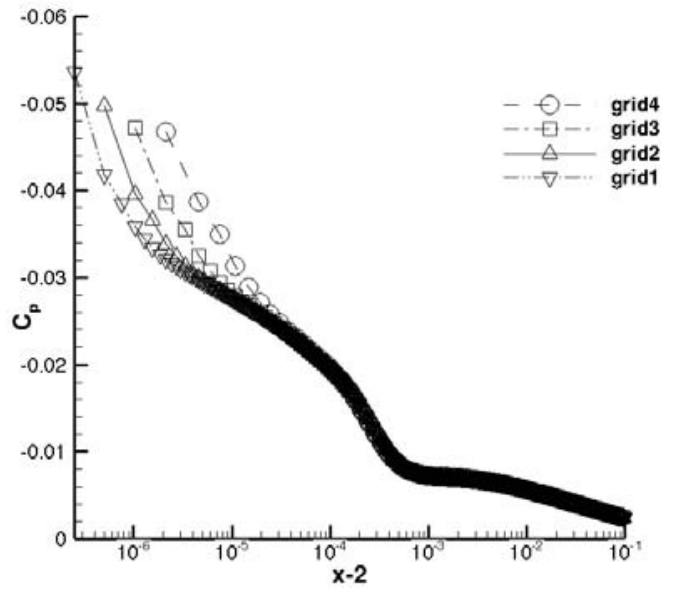

(b) Near trailing edge.

Figure 55. Pressure downstream of the trailing edge.

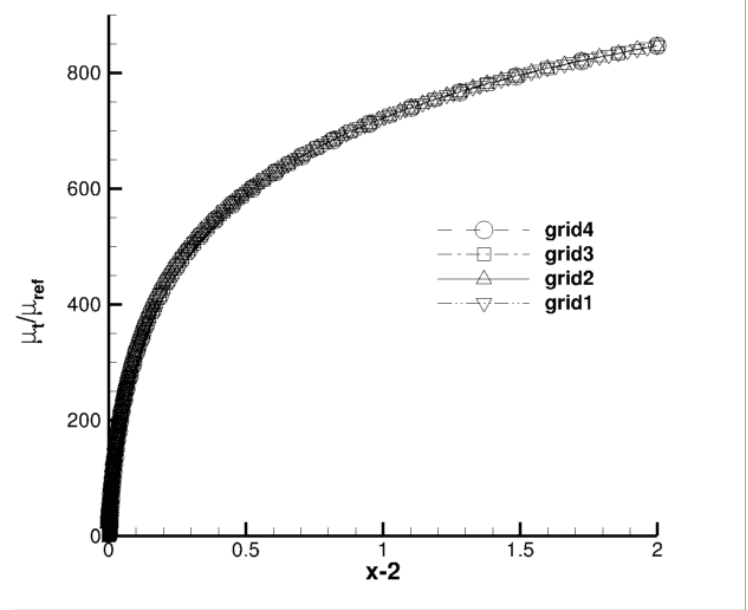

(a) Global view.

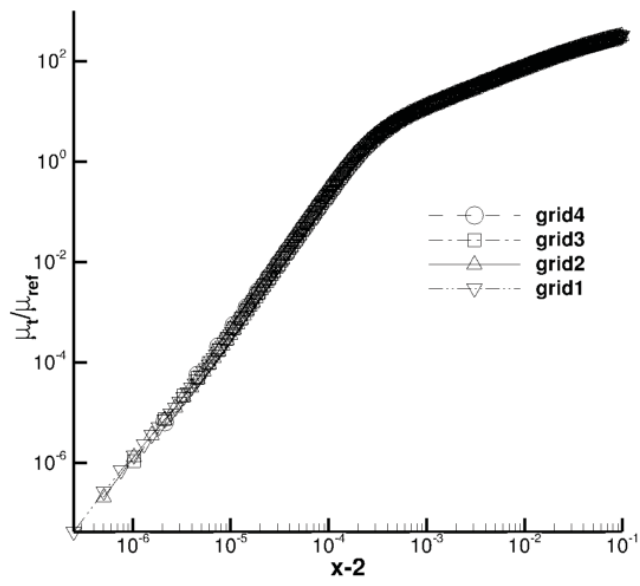

(b) Near trailing edge.

Figure 56. Eddy viscosity downstream of the trailing edge. 


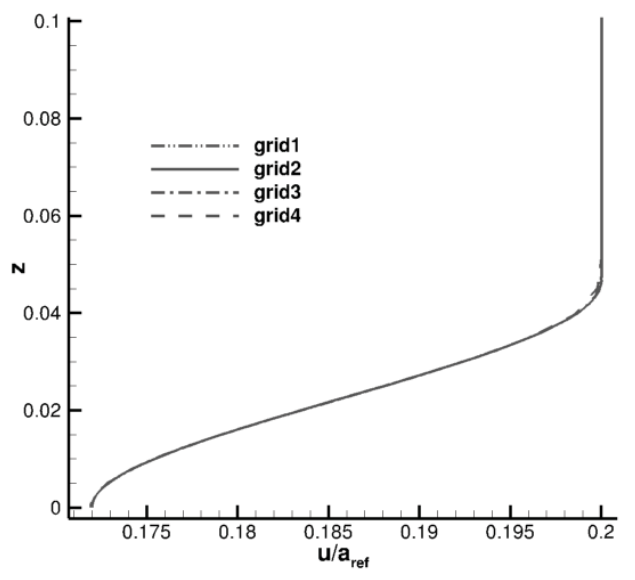

(a) Global view.

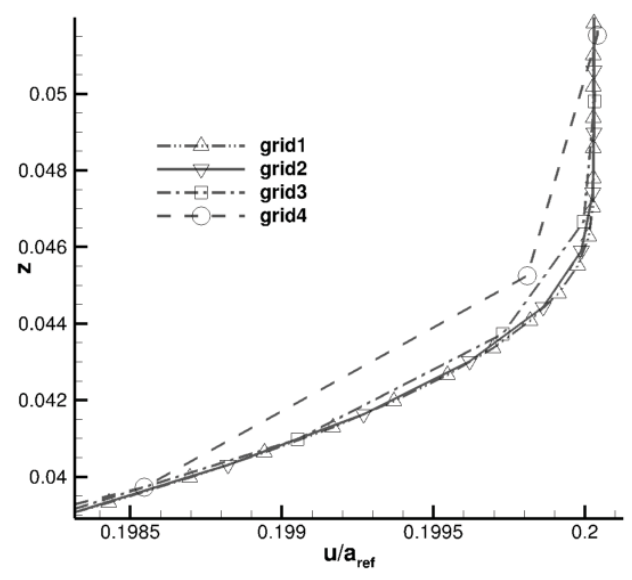

(b) Near edge of wake.

Figure 57. Wake velocity profile at $x=3$.

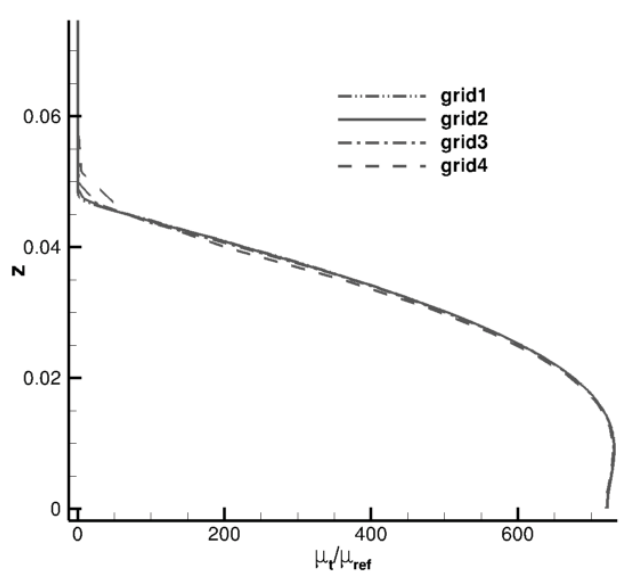

(a) Global view.

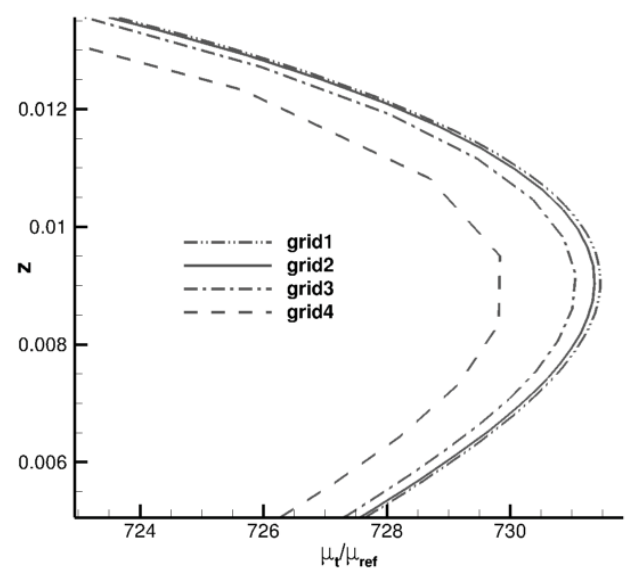

(b) Near maximum eddy viscosity.

Figure 58. Wake eddy viscosity profile at $x=3$. 
nonlinear V-cycle, ${ }^{35} \mathrm{FAS}(2,2)$, with 2 relaxations preceding and 2 relaxations following the coarse-grid correction, is used. Each relaxation uses a tightly-coupled formulation and one multicolor point-implicit sweep and one lineimplicit sweep over the entire domain at each multigrid level. The coarsest grid (level 4) takes just over 100 cycles (400 relaxations) to converge residuals to the residual tolerance of $10^{-12}$. The finer grids take about 20 FAS cycles to converge residuals to the tolerance. The drag coefficient converges faster on grids with $A R_{L E}=1$ than on grids with $A R_{L E}=1200$; specifically, the level of discretization error is reached in just a few cycles on grids with $A R_{L E}=1$ and within ten cycles on grids with $A R_{L E}=1200$. This faster convergence of the drag coefficient is attributed to a better initial approximation provided by the FMG solver from a coarser grid with $A R_{L E}=1$.

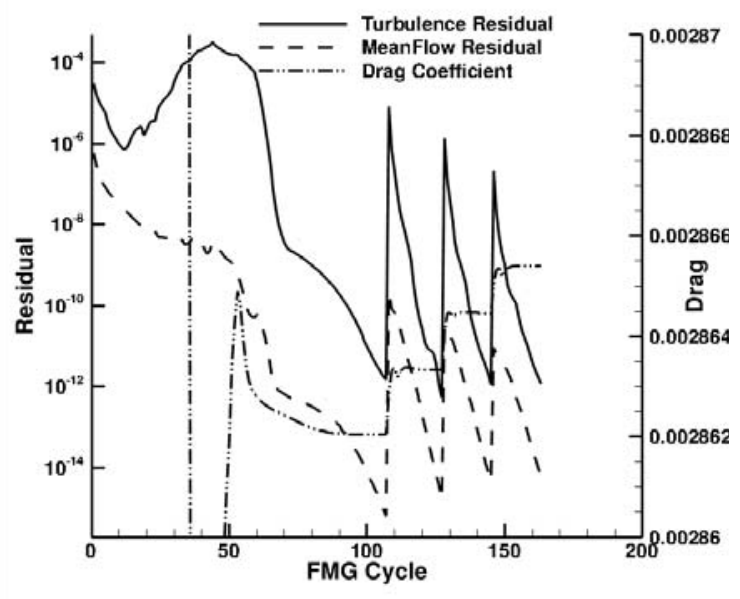

(a) $A R_{L E}=1200$.

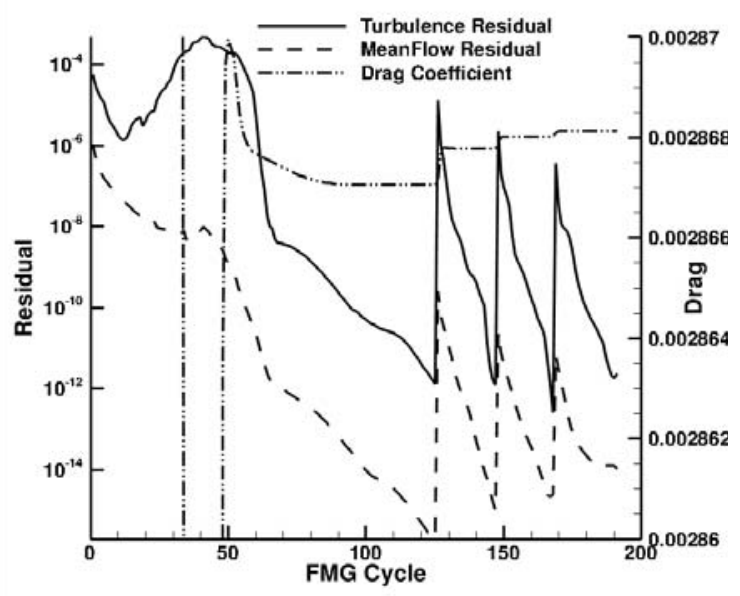

(b) $A R_{L E}=1$.

Figure 59. Iterative convergence of residuals and drag coefficient versus multigrid FMG cycle for 4 finest grids.

\section{F. Variation of the Farfield Boundary Locations}

The effects of upper and upstream/downstream boundary locations have been studied parametrically and the results are shown in Fig. 60. For these studies, the aspect ratio of the grids was $A R_{L E}=1200$. The grids are symmetric about $x=1$, so the distances from the plate to the upstream and downstream boundaries are the same. The drag coefficient and maximum eddy viscosity on the finest grid are shown for varying locations. In Fig. 60 (a), the distance from the plate to the upstream and downstream boundaries is fixed at $1(-1 \leq x \leq 3)$, and the upper boundary is shifted. Both the drag and maximum eddy viscosity vary linearly with respect to the inverse of distance from the plate to the upper boundary. The drag coefficients computed on the domains with upper boundary located at $z=4$ and $z=1$ are 0.015 and 0.055 counts lower, respectively, than the drag coefficient extrapolated to the limit of the boundary at $z=\infty$. Corresponding changes to the maximum eddy viscosity are less than $1 \%$.

In Fig. 60 (b), the upper boundary fixed at $z=4$, and the inflow/outflow boundary locations are shifted. The drag varies linearly with respect to the inverse of distance from the plate to the downstream (or upstream) boundary location. The variation in the computed drag coefficient is smaller $(<0.01 \mathrm{drag}$ count $)$ than the variation due to changes in the upper boundary location. The maximum eddy viscosity increases considerably as the upstream/downstream boundaries are moved farther from the plate, slightly faster than the inverse of the distance to the boundaries. Based of these results, the upper boundary location was chosen as $z=4$ and the horizontal extent of the domain was chosen as $-2 \leq x \leq 4$ to provide the distance of 2 from the plate to the downstream (and upstream) boundary.

\section{Concluding Remarks}

A detailed grid convergence study has been conducted to establish accurate reference solutions corresponding to a one-equation linear eddy-viscosity Spalart-Allmaras (SA) turbulence model for two-dimensional (2D) turbulent flows around the NACA 0012 airfoil and a flat plate configuration. The investigation of the NACA 0012 airfoil involved three widely used computational fluid dynamics (CFD) codes, FUN3D (NASA), CFL3D (NASA), and TAU (DLR), 


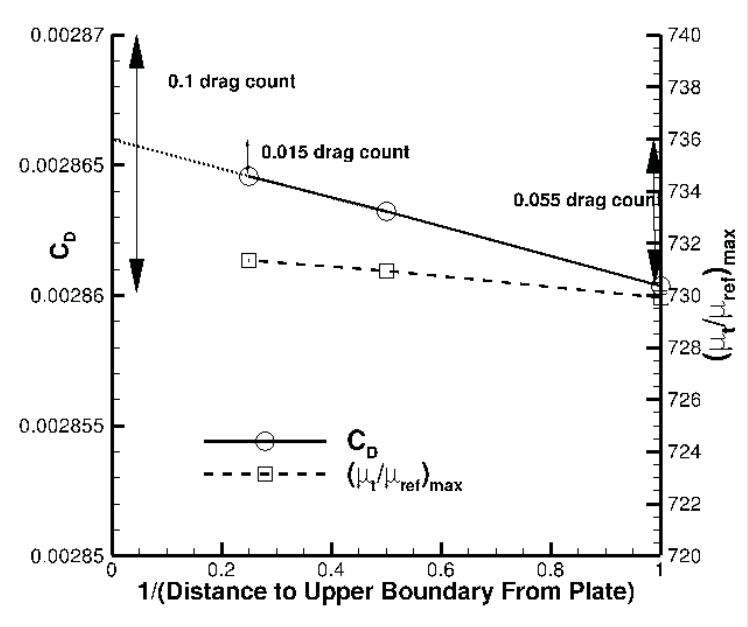

(a) Variation in upper boundary location; distance from plate to downstream boundary of 1 .

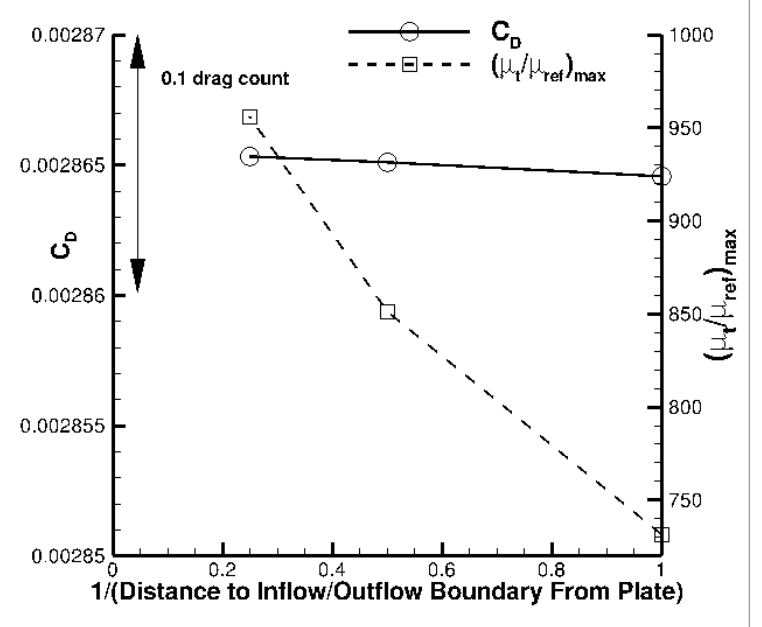

(b) Variation in downstream boundary location; distance from plate to upper boundary of 4 .

Figure 60. Effect of varying boundary locations; $A R_{L E}=1200$; FUN3D finest grid values.

and three families of uniformly refined structured grids with different density distribution. The following observations have been reported.

1. Solutions computed on different grid families appear to converge to the same continuous limit, but exhibit different convergence characteristics.

2. The grid resolution in the vicinity of geometric singularities, such as a sharp trailing edge, is the major factor affecting accuracy of discrete solutions, more prominent than differences in discretization schemes and/or grid elements.

3. On grids from the family with an improved trailing-edge resolution, the solutions obtained with different codes are similar. Plotted on a global scale, the solutions on the finest grid are almost indistinguishable. Differences in the pressure and skin friction coefficients appear only in a narrow range within $0.001 \mathrm{c}$ distance from the trailing edge and near the minimum pressure location in the leading edge. Off-body profiles differ mostly in this narrow range of the trailing edge.

4. The aerodynamic coefficients predicted by the three codes on the finest grid with $15 \mathrm{M}$ degrees of freedom and with an improved trailing-edge resolution show an impressive agreement. The code-to-code variations in the total drag are less than $0.1 \%$ ( 0.1 count), in the lift are less than $0.02 \%$, and in the pitching moment are less than $1 \%$.

5. Even on such fine grids producing such accurate solutions, the asymptotic convergence order has not been established.

Similar observations have been made for FUN3D computations performed for a turbulent flow around a cascade of flat plates: the solution accuracy and convergence have greatly benefited from an improved streamwise resolution near geometric singularities, i.e., the leading and trailing edges. Namely, solutions computed on a sequence of uniformly refined grids with a high resolution near the leading and trailing edges (i.e., with the local aspect ratio of $A R_{L E}=1$ ) show significantly less variation in grid refinement than solutions computed on grids with the same number of degrees of freedom but with a lower resolution near the edges (the local aspect ratio of $A R_{L E}=1200$ ). On grids with $A R_{L E}=1$, the drag contributions from the leading and trailing-edge sections converge with first order. The drag contribution from the middle-plate section converges with an apparent order of 3.3. Skin friction convergence exhibits an order of 1.6 in a fixed location next to the leading edge, an order of 2.5 in the middle of the plate, and a betterthan-first order near the trailing edge. The flow velocity at fixed locations behind the trailing edge converges with an apparent order of 1.6. The local eddy viscosity converges with an apparent second order in the middle of the plate, 
near the trailing edge, and in the wake, but the maximum eddy viscosity converges with approximately first order. On grids with $A R_{L E}=1200$, the skin friction errors are less than those with $A R_{L E}=1$ on coarser grids but the grid convergence order is less than first order. On all grids, the maximum errors in skin friction and surface pressure coefficients always occur over a fixed number of grid points near the leading and/or trailing edges and decay slowly with grid refinement.

Note that on the grids used in the study, convergence of turbulent-flow solutions near geometric singularities is significantly different from the solution for an elliptic (pure diffusion) equation. The elliptic-equation solution exhibits first-order convergence on uniformly refined grids at any fixed interior location. Observed convergence of the turbulent-flow solutions does not degrade to the same degree. This difference in convergence may indicate that, in spite of a high solution accuracy obtained on the grids used in the study, much finer grids are needed to observe the expected first-order asymptotic convergence order. This observation is discouraging to the prospects of realizing high asymptotic convergence orders on uniformly refined grids. The topic deserves further investigation. A corner (nonuniform) refinement strategy is shown in the appendix to recover design order convergence for the elliptic-equation solution. Its success in application to the pure-diffusion equation provides an indication that improved resolution near geometric singularities is essential to improve the accuracy of turbulent-flow simulations.

\section{Acknowledgments}

The first author acknowledges support from NASA Cooperative Agreement NNL09AA00A.

\section{References}

${ }^{1}$ Rumsey, C. L., "Turbulence Modeling Resource Website," http: / / turbmodels. larc. nasa.gov [retrieved 18 Nov. 2014].

${ }^{2}$ Rumsey, C. L., Smith, B. R., and Huang, G. P., "Description of a Website Resource for Turbulence Modeling Verification and Validation," AIAA Paper 2010-4742.

${ }^{3}$ Spalart, P. R. and Allmaras, S. R., "A One-Equation Turbulence Model for Aerodynamic Flows," Recherche Aerospatiale, No. 1, 1994, pp. $5-21$

${ }^{4}$ Guide for Verification and Validation of Computational Fluid Dynamics Simulations, AIAA G-007-1998(2002).

${ }^{5}$ Oberkumpf W. L. and Roy C. J., Verification and Validation in Scientific Computing, Cambridge University Press, Cambridge, 2010.

6 "Fifth AIAA CFD Drag Prediction Workshop Website," http://aaac.larc.nasa.gov/tsab/cfdlarc/aiaa-dpw [retrieved 18 Nov. 2014]

${ }^{7}$ Levy, D., Laflin, K., Vassberg, J., Tinoco, E., Mani, M., Rider, B., Brodersen, O., Crippa, S., Rumsey, C., Wahls, R., Morrison, J., and amd M. Murayama, D. M., "Summary of Data from the Fifth AIAA CFD Drag Prediction Workshop," AIAA Paper 2013-0046.

${ }^{8}$ Nishikawa, H., Diskin, B., Thomas, J. L., and Hammond, D. P., "Recent Advances in Agglomerated Multigrid," AIAA Paper $2013-863$.

${ }^{9}$ Diskin, B. and Nishikawa, H., "Evaluation of Multigrid Solutions for Turbulent Flows," AIAA Paper 2014-082.

${ }^{10}$ Langer, S., Schwöppe, A. E., and Kroll, N., "The DLR Flow Solver TAU - Status and Recent Algorithmic Developments," AIAA Paper 2014-0080.

${ }^{11}$ Laarsonen, P., "On the Discretization Error of the Dirichlet Problem in a Plane Region with Corners," Annales Academi Scientiarum Fennic Mathematica, Vol. 408, 1967, pp. 1-16.

${ }^{12}$ Rumsey, C. L., "CFL3D Contribution to the AIAA Supersonic Shock Boundary Layer Interaction Workshop," NASA/TM 2010-216858.

${ }^{13}$ Heeg, J., "Overview and Lessons Learned from the Aeroelastic Prediction Workshop," AIAA Paper 2013-1798.

${ }^{14}$ Park, M. A., Lee-Rausch, E. M., and Rumsey, C. L., "FUN3D and CFL3D Computations for the First High Lift Prediction Workshop," AIAA Paper 2011-0936.

${ }^{15}$ Park, M. A., Laflin, K. R., Chaffin, M. S., Powell, N., and Levy, D. W., "CFL3D, FUN3D, and NSU3D Contributions to the Fifth Drag Prediction Workshop," Journal of Aircraft, Vol. 51, No. 4, 2014, pp. 1268-1283.

${ }^{16}$ Anderson W. K., Thomas, J. L., and Van Leer, B., "Comparison of Finite Volume Flux Vector Splittings for the Euler Equations," AIAA Journal, Vol. 24, No. 9, 1986, pp. 1453-1460.

${ }^{17}$ Burg C. O. E., "Higher Order Variable Extrapolation For Unstructured Finite Volume RANS Flow Solvers," AIAA Paper 2005-4999.

${ }^{18}$ Allmaras, S. R., Johnson, F. T., and Spalart, P. R., "Modifications and Clarifications for the Implementation of the Spalart-Allmaras Turbulence Model," Seventh International Conference on Computational Fluid Dynamics, Big Island, Hawaii, 2012.

${ }^{19}$ E. M. Lee-Rausch, C. L. Rumsey, M. A. P., "Grid-Adapted FUN3D Computations for the Second High Lift Prediction Workshop," AIAA Paper 2014-2395.

${ }^{20}$ Bartels, R., Vatsa, V. N., Carlson, J.-R., and Mineck, R., "FUN3D Grid Refinement and Adaptation Studies for the Ares Launch Vehicle," AIAA Paper 2010-4372.

${ }^{21}$ Chwalowski, P. and Heeg, J., "FUN3D Analysis in Support of the First Aeroelastic Prediction Workshop," AIAA Paper 2013-0785.

${ }^{22}$ Park, M. A., "Low Boom Configuration Analysis with FUN3D Adjoint Simulation Framework," AIAA Paper 2011-3337.

${ }^{23}$ Gnoffo, P. A., Wood, W. A., Kleb, B., Alter, S. J., Padilla, J., and White, J. A., "Functional Equivalence Acceptance Testing of FUN3D for Entry, Descent, and Landing Applications," AIAA Paper 2013-2558.

${ }^{24}$ Vatsa, V. N., Khorrami, M. R., Park, M. A., and Lockard, D. P., "Aeroacoustic Simulation of Nose Landing Gear on Adaptive Unstructured Grids with FUN3D," AIAA Paper 2013-2071. 
${ }^{25}$ Nielsen, E. J. and Diskin, B., "Discrete Adjoint-Based Design for Unsteady Turbulent Flows on Dynamic Overset Unstructured Grids," AIAA J., Vol. 51, No. 6, 2013, pp. 1355-1373.

${ }^{26}$ Schwamborn, D., Gerold, T., and Heinrich, R. K., "The DLR TAU-Code: Recent Applications in Research and Industry," Proceedings of ECCOMAS CFD 2006, Delft, The Netherlands, 2006.

${ }^{27}$ Heinrich, R. K., Kroll, N., Neumann, J., and Nagel, B., "Fluid-Structure Coupling for Aerodynamic Analysis and Design,” AIAA Paper $2008-0561$.

${ }^{28}$ Nishikawa, H., "Beyond Interface Gradient: A General Principle for Constructing Diffusion Schemes," AIAA Paper 2010-5093.

${ }^{29}$ Eisfeld, B., "Numerical Simulation of Aerodynamic Problems with a Reynolds Stress Turbulence Model," New Results in Numerical and Experimental Fluid Mechanics V, Vol. 92 of Notes on Numerical Fluid Mechanics and Multidisciplinary Design, Springer, Berlin, Germany, 2006, p. 413421.

${ }^{30}$ Dwight, R. P., "A Comparison of Implicit Algorithms for the Navier-Stokes Equations on Unstructured Grids," Third International Conference on Computational Fluid Dynamics,Toronto, Canada, 2004.

${ }^{31}$ Thomas, J. L. and Salas, M. D., "Far-Field Boundary Conditions for Transonic Lifting Solutions to the Euler Equations," AIAA Journal, Vol. 24, No. 7, 1986, pp. 1074-1080.

${ }^{32}$ Anderson, W. K., Private Communication, 2014.

${ }^{33}$ Darmofal, D. L. and Allmaras S. R.*, Private Communication, 2014.

${ }^{34}$ White, F. M., Viscous Fluid Flow, McGraw-Hill, 2nd ed., 1991.

${ }^{35}$ Trottenberg, U., Oosterlee, C. W., and Schüller, A., Multigrid, Academic Press, 2000.

${ }^{36}$ Thomas, J. L., Diskin, B., and Rumsey, C. L., "Towards Verification of Unstructured Grid Methods," AIAA J., Vol. 46, No. 12, 2008, pp. 3070-3079.

${ }^{37}$ Yano, M., and Darmofal, D. “An optimization-based framework for anisotropic simplex mesh adaptation,” J. Comp. Phys., Vol. 231, 2012, pp. 7626-7649.

${ }^{38}$ Yano, M. "An optimization framework for adaptive higher-order discretizations of partial differential equations on anisotropic simplex meshes," Ph.D. thesis, Massachusetts Institute of Technology, Dept. of Aeronautics and Astronautics, Jun. 2012.

\section{Appendix: Elliptic Equation with Reentrant Corner Singularity}

The long-known accuracy degeneration in solutions of elliptic equations on domains with reentrant corner singularities ${ }^{11}$ is revisited. The reentrant corner geometry is represented parametrically by an exterior angle, $\omega$, that can be selected to match the local trailing-edge geometry of the NACA 0012 airfoil. The computations shown in this appendix are for $\omega=2 \pi$, which corresponds to a cusped trailing-edge geometry and the most severe accuracy degradation. Elliptic equations describe diffusion phenomena and thus applies directly to Stokes flows. The relevance of the accuracy degeneration to high Reynolds number flows has not been studied.

The exact solution, $q$, of the 2D Laplace equation,

$$
\partial_{x x} q+\partial_{y y} q=0
$$

satisfying homogeneous Dirichlet boundary conditions for a reentrant corner situated on the left of the computational domain (as in Fig. 61 (a)) is given by the following,

$$
q=\hat{r}^{\hat{\alpha}} \sin \left(\hat{\alpha} \theta^{L}\right),
$$

where the angle $\theta^{L}$ increases clockwise with a branch cut along $\theta=\pi$,

$$
\theta^{L}=\pi-\theta .
$$

The position of the reentrant corner tip (also referred as the trailing edge by analogy with the NACA 0012 geometry) is $\left(x_{T E}, z_{T E}\right)=(1,0)$, the inverse relative exterior angle is $\hat{\alpha} \equiv \pi / \omega$, and

$$
\begin{gathered}
\hat{x}=x-x_{T E}, \\
\hat{z}=z-z_{T E}, \\
\theta=\arctan 2(\hat{z}, \hat{x}), \\
\hat{r}=\sqrt{\hat{x}^{2}+\hat{z}^{2}} .
\end{gathered}
$$

Dirichlet conditions, $q=0$, are applied for $x \leq x_{T E}$ along the radial lines $\theta=\pi-(2 \pi-\omega) / 2$ and $\theta=-\pi+(2 \pi-$ $\omega) / 2$. The exact solution contours with $\omega=2 \pi$ are shown in Fig. 61 (a). For completeness, the exact solution with the reentrant corner situated on the right of the domain, corresponding to the geometry most often cited in the literature, ${ }^{35}$ is given by the following,

$$
q=\hat{r}^{\hat{\alpha}} \sin \left(\hat{\alpha} \theta^{R}\right),
$$


where the angle $\theta^{R}$ increases counter-clockwise with a branch cut along $\theta=0$,

$$
\theta^{R}= \begin{cases}\theta+2 \pi & \text { if } \hat{z}<0, \\ \theta & \text { otherwise }\end{cases}
$$

The theoretical estimates ${ }^{11}$ for convergence orders of discretization error, $\epsilon$, on uniformly refined Cartesian grids are

$$
\begin{aligned}
& \|\epsilon\|_{\infty} \approx h^{\hat{\alpha}}, \\
& \|\epsilon\|_{1} \approx h^{2 \hat{\alpha}} .
\end{aligned}
$$

Here, $\|\cdot\|_{\infty}$ and $\|\cdot\|_{1}$ are the $L_{\infty}$ and $L_{1}$ norms, respectively.

The lowest convergence order is predicted for a cusped trailing edge, which is a zero-angle reentrant corner $(\omega=2 \pi$, $\hat{\alpha}=0.5)$,

$$
\begin{aligned}
& \|\epsilon\|_{\infty} \approx \sqrt{h}, \\
& \|\epsilon\|_{1} \approx h .
\end{aligned}
$$

This is a significant global degradation of second-order convergence expected for nominally second-order discretization schemes and smooth solutions.

To demonstrate and repair this degradation, several sequences of rectilinear grids are generated on the domain $(x, z) \in[0,2] \times[-1,1]$ centered about $\left(x_{T E}, z_{T E}\right)$. Solutions are computed on a sequence of uniformly-refined isotropic Cartesian grids as well as on sequences of non-uniformly refined rectilinear grids with additional degrees of freedom added near the reentrant corner. The latter refinement is reference as corner refinement to distinguish it from uniform refinement.

A 2D rectilinear grid is derived from a one-dimensional (1D) primal mesh generated on the interval $0 \leq x \leq 1$. This $1 \mathrm{D}$ mesh is mapped symmetrically on the interval $1 \leq x \leq 2$ and also on the $z$-axis. The 2D mesh is constructed as the tensor product of the 1D $x$ - and $z$-directional meshes (see Fig. 61 (b) and (c)). A sequence of uniform nested 1D meshes on the primal interval leads to a sequence of uniformly refined isotropic Cartesian grids.

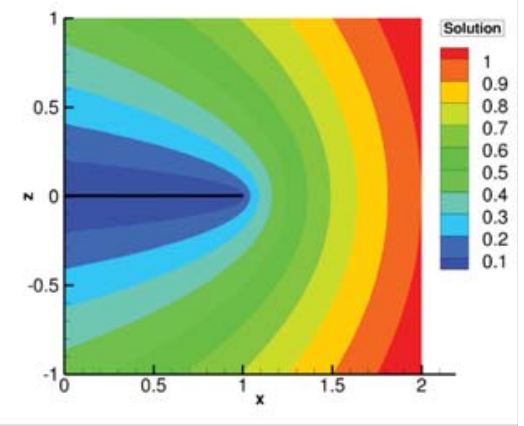

(a) Exact solution. The reentrant-corner boundary is the black line on the left side $(x \leq 1, z=0)$.

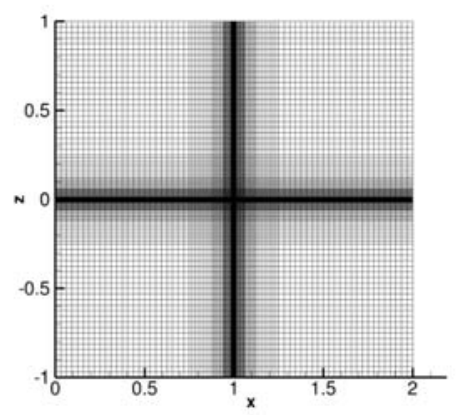

(b) Corner-Refinement grid (level 4; 2 seed cells).

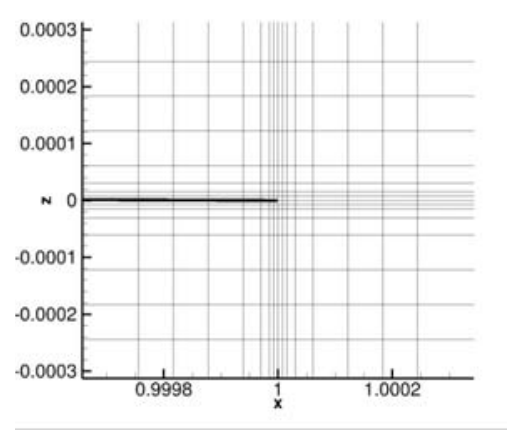

(c) Corner-Refinement grid near reentrant corner (level 4; 2 seed cells).

Figure 61. Reentrant corner: exact solution and grids.

The starting coarse grid for a sequence of corner-refined rectilinear grids is one of the Cartesian grids characterized by the number mesh spacings (cells) over the interval $0 \leq x \leq 1$; this number is referred as the number of seed cells. One step of the grid refinement is defined as follows. First, all coarse cells except the reentrant-corner cell (the cell attached to the $\left(x_{T E}, z_{T E}\right)$ node) are divided into two equal fine cells. The coarse reentrant-corner cell undergoes the corner refinement. It is subdivided 4 times; in each subdivision, only the local cell closest to the reentrant corner is divided into two equal cells. Thus, the original coarse reentrant-corner cell has been divided into four cells, while each of the other coarse cells has been divided into two cells. If the size of the coarse reentrant-corner cell is defined by $s=1$, then the sizes of the four fine cells are $s=\{s / 2, s / 4, s / 8, s / 16, s / 16\}$, where the two smallest spacings are nearest to the reentrant corner. Note that the mesh size away from the reentrant-corner has been uniformly reduced by 
factor 2; the mesh size next to the reentrant corner has been reduced by factor 16 . The further mesh refinement on the interval $0 \leq x \leq 1$ is done recursively.

The mesh size, $h$, in the theoretical estimate of Eq. 1 is the local mesh size in a vicinity of the reentrant corner. In the corner refinement, the square root of the local mesh size is proportional to the square of the mesh size in the uniformly refined part of the domain. This corner refinement is expected to recover second-order convergence of discretization errors for the worst case of $\omega=2 \pi$.

The number of seed cells effectively determines the physical extent of the grid affected by the corner refinement; the rest of the computational domain undergoes the uniform grid refinement. A grid started from 2 seed cells (level 1) and refined through 3 steps of corner refinement (to level 4) is shown in Fig. 61 (b) and (c). The grid differs from a corresponding uniformly refined grid over $\approx 44$ percent of the domain. For example, the upper left quadrant defined by $x \leq 0.75$ and $z \geq 0.25$ is uniformly refined. A corner-refinement grid started from 16 seed cells (not shown) differs from a uniformly refined over a much smaller region $(\approx 6$ percent $)$ of the physical domain; the upper left quadrant defined by $x \leq 0.9675$ and $z \geq 0.0325$ is uniformly refined.

The degrees of freedom on the uniform and corner-refinement grids are shown in Tables 4 and 5 for grid sequences started from 2 and 16 seed cells, respectively. The degrees-of-freedom increase from level to level is also tabulated for the corner refinement. Although the increase factor is quite large for coarse-grid levels, the factor asymptotes to the factor of four, which is typical for the uniform refinement. Note that the uniform-refinement grid levels in the tables are not synchronized; level 2 from Table 4 corresponds to level 1 from Table 5.

Table 4. Number of degrees of freedom on the uniform and corner-refinement grids with 2 seed cells. Number in parentheses is the degrees-of-freedom increase factor from the previous level.

\begin{tabular}{lrr}
\hline Grid Level & Uniform-Refinement & Corner-Refinement \\
\hline \hline 8 (coarsest) & 27 & 27 \\
7 & 85 & $232(8.59)$ \\
6 & 297 & $1242(5.35)$ \\
5 & 1105 & $5662(4.56)$ \\
4 & 4257 & $24102(4.26)$ \\
3 & 16705 & $99382(4.12)$ \\
2 & 66177 & $403542(4.06)$ \\
1 (finest) & 263425 & $1626262(4.03)$ \\
\hline
\end{tabular}

Table 5. Number of degrees of freedom on the uniform and corner-refinement grids with $\mathbf{1 6}$ seed cells. Number in parentheses is the degrees-of-freedom increase factor from the previous level.

\begin{tabular}{lrr}
\hline Grid Level & Uniform-Refinement & Corner-Refinement \\
\hline \hline 4 (coarsest) & 1105 & $1105(\mathrm{NA})$ \\
3 & 4257 & $5076(4.59)$ \\
2 & 16705 & $21682(4.27)$ \\
1 (finest) & 66177 & $89550(4.13)$ \\
\hline
\end{tabular}

The discretization errors on uniformly refined grids at $z=0$ and $x \geq 1$ are shown in Fig. 62. The finest grid denoted as "Grid 1" in the figure corresponds to the level 1 grid in Table 4 . The reentrant corner position is at $x=1$. The same errors on a logarithmic scale are shown versus linearly scaled $x$ (Fig. 62 (a)) and versus the logarithm of $x-1$ (Fig. 62 (b)); the latter emphasizes errors near the reentrant corner. As expected, the discretization error shows first-order convergence at a fixed distance from the corner. The maximum error occurs at the first point away from the corner and converges with a $\sqrt{h}$ order.

The discretization errors on corner-refinement grids at $z=0$ and $x \geq 1$ are shown in Fig. 63 and 64 for 2 and 16 seed cells, respectively. For both sequences of corner-refinement grids, the discretization error shows uniform secondorder convergence - at all fixed distances from the corner and in the maximum, which again occurs at the first point away from the corner. 


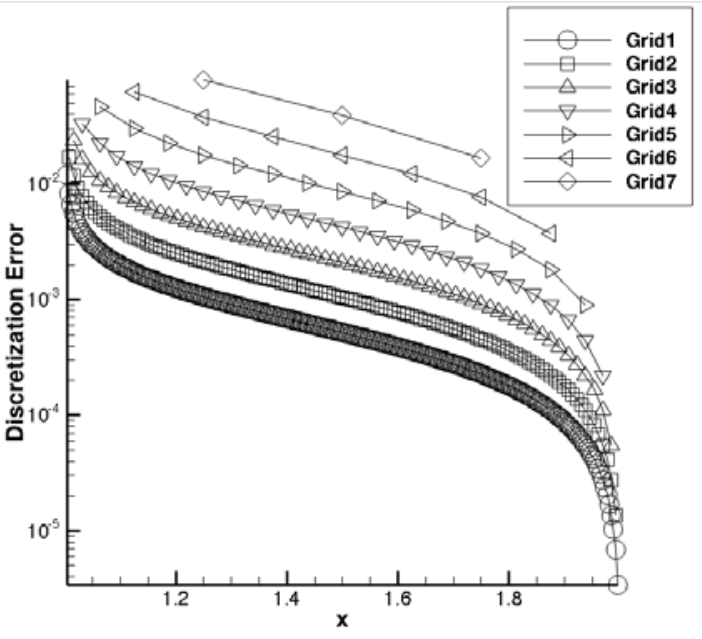

(a) Linear scale in $x$.

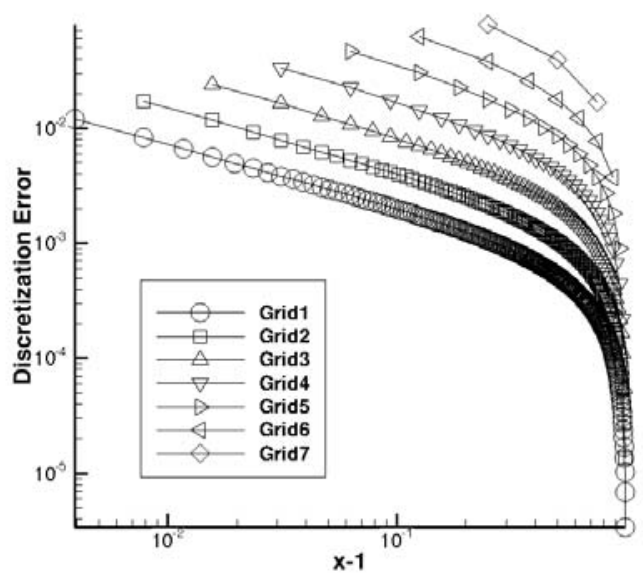

(b) Logarithmic scale in $x-1$.

Figure 62. Discretization errors in grid refinement at $z=0$ and $x \geq 1$ for uniform grid.

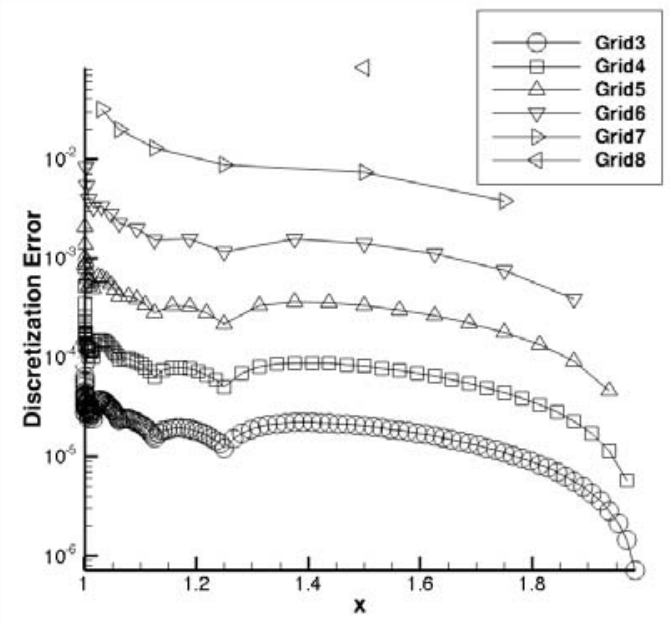

(a) Linear scale in $x$.

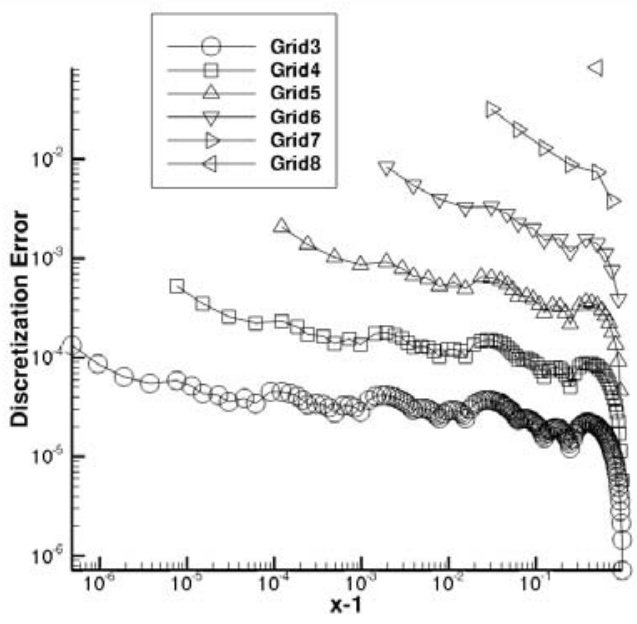

(b) Logarithmic scale in $x-1$.

Figure 63. Discretization errors in grid refinement at $z=0$ and $x \geq 1$ for corner-refinement grids formed from 2 seed cells. 


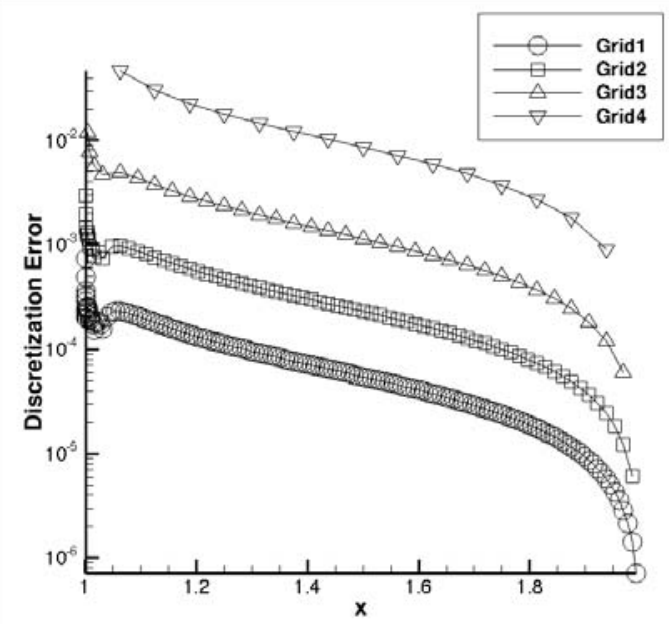

(a) Linear scale in $x$.

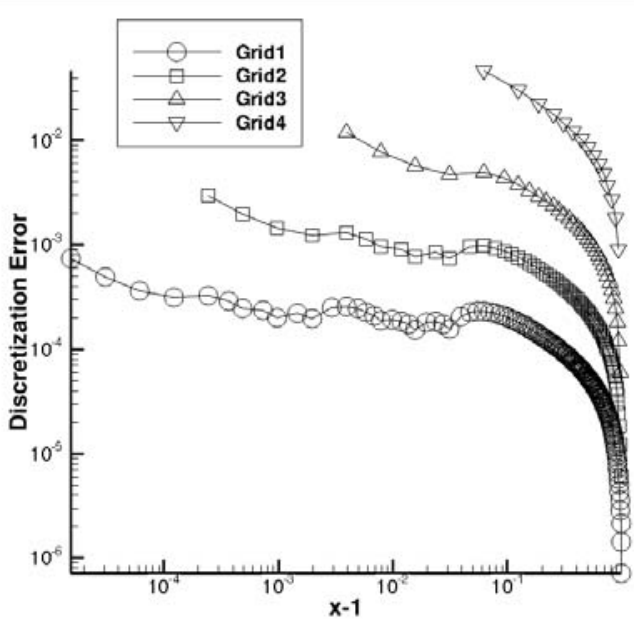

(b) Logarithmic scale in $x-1$.

Figure 64. Discretization errors in grid refinement at $z=0$ and $x \geq 1$ for corner-refinement grids formed from 16 seed cells

The local discretization errors at $x=1.5$ and $z=0$ for uniform and corner-refinement grids are shown in Fig. 65 . The logarithm of the errors are shown versus the logarithm of $h_{\max }$ in Fig. 65 (a) and versus the logarithm of an effective $h$ based on the number of degrees of freedom, $h_{e f f} \equiv N^{-1 / 2}$, in Fig. 65 (b). Triangles indicating the firstand second-order convergence slopes are shown for reference. Computations on the uniform grids exhibit first-order convergence and computations on corner-refinement grids exhibit second-order convergence on finer grids. The errors reduce immediately on both corner-refinement sequences. At fixed degrees of freedom, $N$, the corner-refinement errors with 2 seed cells (corner refinement affects $44 \%$ of the domain) are less than the errors with 16 seed cells (corner refinement affects $6 \%$ of the domain).

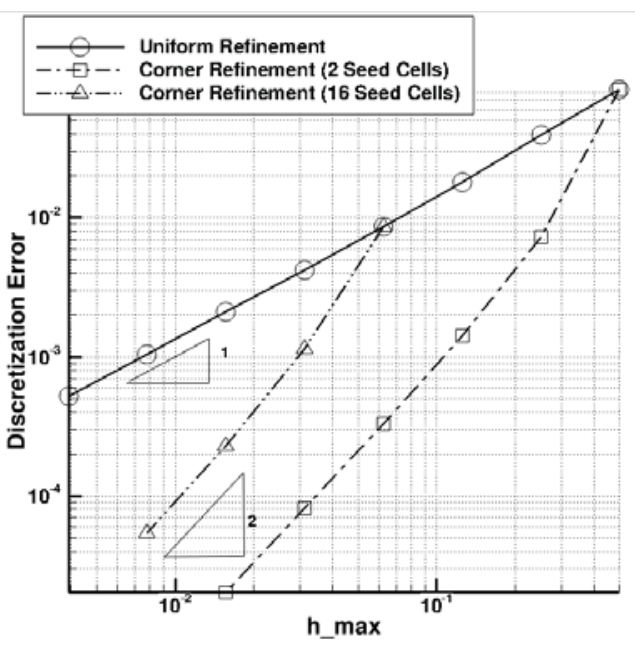

(a) Discretization error convergence versus $h_{\max }$.

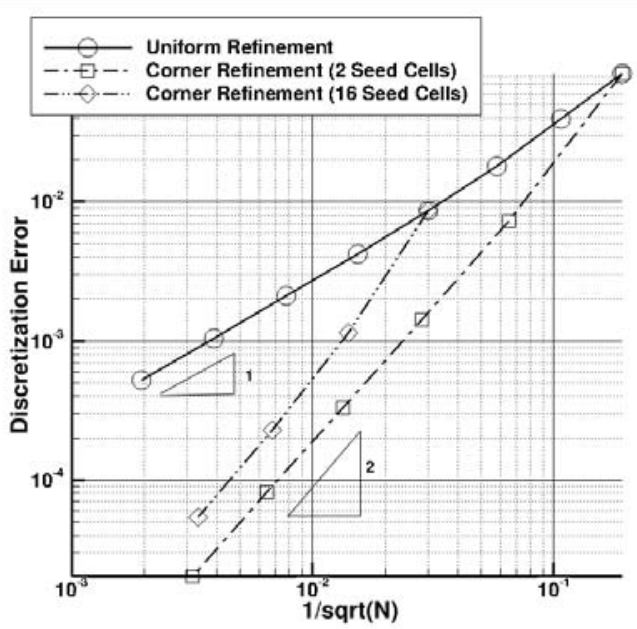

(b) Discretization error convergence versus $h_{\text {eff }}$.

Figure 65. Convergence of discretization errors at $x=1.5$ for uniform and non-uniformly refined grids .

A parametric study for the corner refinement method has been conducted on a computational domain including only the right half of the domain shown in Fig. 61 (a). Dirichlet boundary conditions are prescribed at $x=1,-1 \leq$ $z \leq 1$. The number of recursive cell divisions is varied from two to four; corresponding smallest mesh size obtained 
after one step of mesh refinement is $1 / 4 s, 1 / 8 s$, and $1 / 16 s$, respectively, where $s$ is the size of the coarse corner cell. The discretization errors on uniform grids and on grids with corner refinement are shown in Fig. 66. The convergence order of the maximum errors (Fig. 66 (a)) improves to the first order with only two recursive cell divisions, but four cell divisions are needed to obtain second-order convergence. The $L_{1}$ norm of discretization errors (Fig. 66 (b)) converges with (nearly) second order with two recursive cell divisions.

The computations indicate that second-order convergence of discretizations errors in all norms can be recovered on non-uniformly corner-refined grids with an increased refinement rate in the vicinity of the geometric singularity. This refinement is a consistent refinement ${ }^{36}$ ensuring that every cell is refined with a rate greater or equal to the rate corresponding to the uniform refinement. Although not shown, similar degradation of convergence order to $O(\sqrt{h})$ locally and to $O(h)$ globally has been observed on uniformly refined grids for higher-order discretization methods. ${ }^{32}$ To recover design-order convergence for high-order methods on domains with geometric singularities, more local subdivisions may be required. To recover design $p$-th order convergence of the $L_{\infty}$ norm of the discretization error, the estimated number of subdivisions is $2 p$, i.e., each corner cell should be divided into $2 p+1$ cells in each dimension. For design-order convergence of the $L_{1}$ norm, $p$ subdivisions should suffice. Grids used in the corner refinement employ more degrees of freedom than corresponding uniformly refined grids, but asymptotically the total number of degrees of freedom increases with the same rate as on uniform-refinement grids. The rectilinear grids used for the corner refinement place many additional degrees of freedom away from the geometric singularity. These remote degrees of freedom seem wasteful; a local adaptive grid refinement is expected to be a much more efficient way to improve convergence and to recover the design-order accuracy.

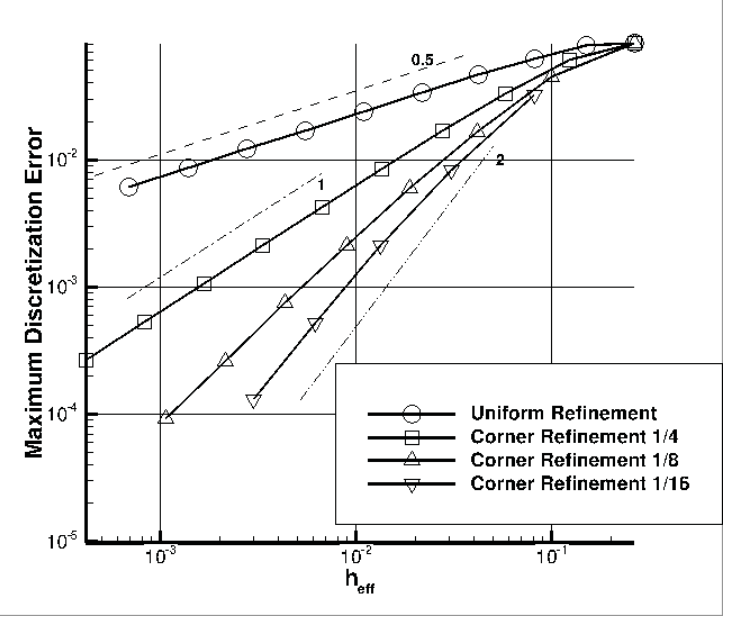

(a) $L_{\infty}$ norm.

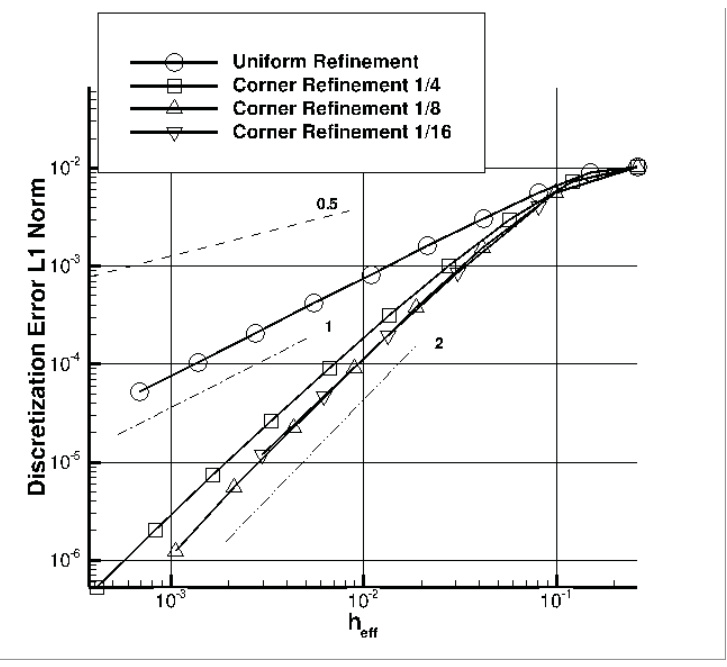

(b) $L_{1}$ norm.

Figure 66. Discretization error for reentrant corner with uniform grids and grids with varying corner refinements; $\omega=2 \pi$.

The corner-refinement grids used herein are closely related to the optimal meshes developed by Yano and Darmo$\mathrm{fal}^{37,38}$ for a series of $L_{2}$ error control problems. The optimal meshes are derived using established techniques based on the polynomial interpolation theory and calculus of variations. For the reentrant-corner problem considered here, the optimal meshes are characterized as

$$
h=C \hat{r}^{k},
$$

where $C$ is a non-zero constant, $\hat{r}$ is the distance from the corner, and $k$ is a grading coefficient given by

$$
k=1-\frac{\hat{\alpha}+1}{p+1} .
$$

The grading coefficient characterizes mesh size disparity. A uniform mesh corresponds to $k=0$, and the grading becomes stronger ( $k$ approaching 1 ) as $\hat{\alpha}$ decreases or $p$ increases. Note that $k=0.5$ for second-order convergence $(p=2)$ and a zero-angle reentrant corner $(\hat{\alpha}=0.5)$.

A 1 D primal mesh on the interval $0 \leq x \leq 1$ can be generated with this gradation using the 1D mapping

$$
\hat{r}=\xi^{\frac{1}{1-k}}
$$


where $\hat{r}=1-x$ is the distance from the corner, and $\xi$ is a mapping coordinate, $0 \leq \xi \leq 1$. Discrete meshes are obtained using equal spacing in the mapping coordinate,

$$
x_{i}=1-\xi_{i}^{\frac{1}{1-k}}, \quad \xi_{i}=\frac{i-1}{N-1} \quad \text { for } \quad \mathrm{i}=1,2, \ldots, \mathrm{N}
$$

where $N$ is the number of nodes on the 1D interval. The local average mesh spacing and stretching factor of two optimal mesh sequences are shown in Figs. 67 and 68. The gradation parameters $k=0.50$ and $k=0.75$ correspond to $p=2$ and $p=5$, respectively, for $\hat{\alpha}=0.5$. The average mesh spacing, $h_{a v g}$, and the stretching factor, $\beta$, are defined pointwise as

$$
\left(h_{a v g}\right)_{i}=\frac{x_{i+1}-x_{i-1}}{2}, \quad \beta_{i}=\frac{x_{i+1}-x_{i}}{x_{i}-x_{i-1}} \quad \text { for } \quad \mathrm{i}=2, \ldots, \mathrm{N}-1
$$

The mesh sequences are obtained by doubling the number of cells in each finer grid. Both mesh sequences corresponding to $k=0.50$ and $k=0.75$ are consistent, i.e., the mesh spacing at any location goes to zero in the limit as $N$ goes to infinity. Also, at a fixed non-zero distance from the corner, the stretching factor goes to unity in the limit as $N$ goes to infinity, and the mesh spacing reduces by a factor of two in each refinement. However, the stretching factor $\beta_{2}$ corresponding to the interior point nearest to the corner does not approach unity in mesh refinement, being 3 and 15, respectively, as shown in Figs. 67 and 68. These fixed stretching factors are a consequence of the singularity in the mapping at $\xi=0$.

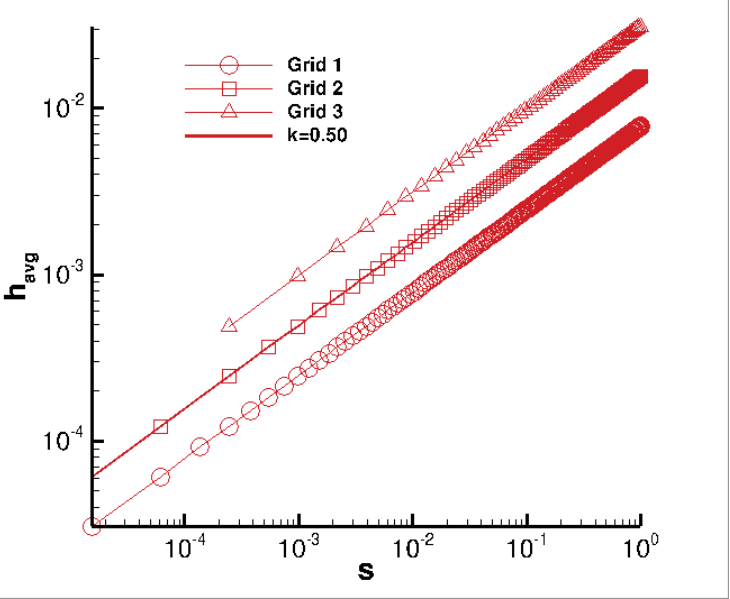

(a) Average mesh spacing

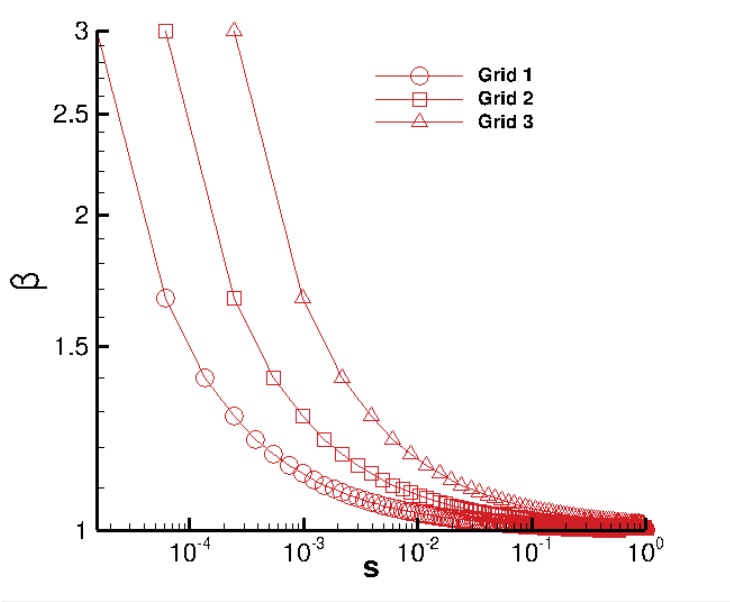

(b) Stretching ratio.

Figure 67. Sequence of grids with optimal grading of Yano and Darmofal; $k=0.5 ; s=1-x$

The corner-refinement grids are characterized on average by a grading $h=C \hat{r}^{1-1 / n}$, where $n$ is the number of recursive subdivisions. Similar to the optimal meshes, the corner-refinement mesh sequences are consistent. The grids are irregular — the stretching factors vary between one and two in the interior. The corner-refinement grading with $n=2$ is similar to the grading of the the optimal meshes with $k=0.5(p=2)$; likewise, the corner-refinement grading with $n=4$ is similar to the grading of the optimal meshes with $k=0.75(p=5)$.

Particular corner-refinement and optimal grids are compared in Fig. 69 (a) and (b) for grading coefficients of $k=0.5$ and $k=0.75$, respectively, for a comparable number of nodes. The corner-refinement grids start from 2 seed cells and have a a portion of the domain that is uniformly refined. The optimal grids are smoother because they are mapped grids and provide a slightly lower minimum spacing for a fixed number of nodes $N$. Although not shown, we have verified that the optimal meshes for $k=0.5$ recover discretization-error convergence of second order in the $L_{1}$ norm, but only first order in the $L_{\infty}$ norm. Computations using optimal meshes with the higher gradation, $k=0.75$, are expected to recover the second-order convergence in all norms. 


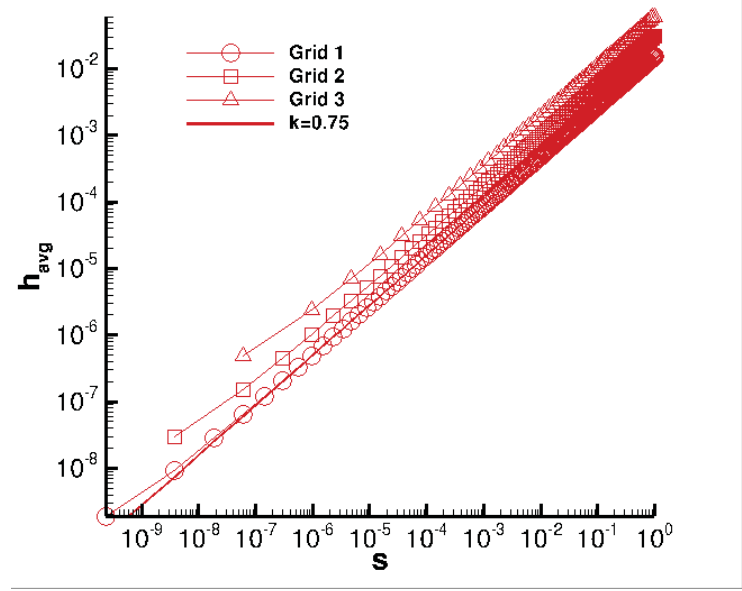

(a) Average mesh spacing.

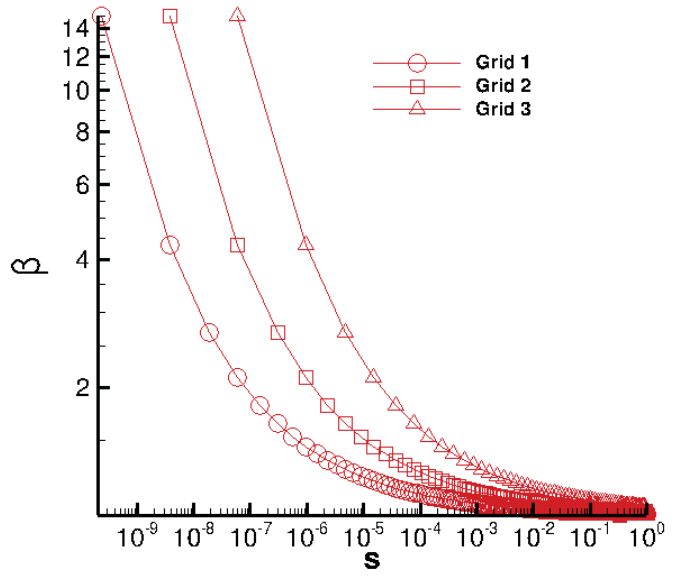

(b) Stretching ratio.

Figure 68. Sequence of grids with optimal grading of Yano and Darmofal; $k=0.75 ; s=1-x$.

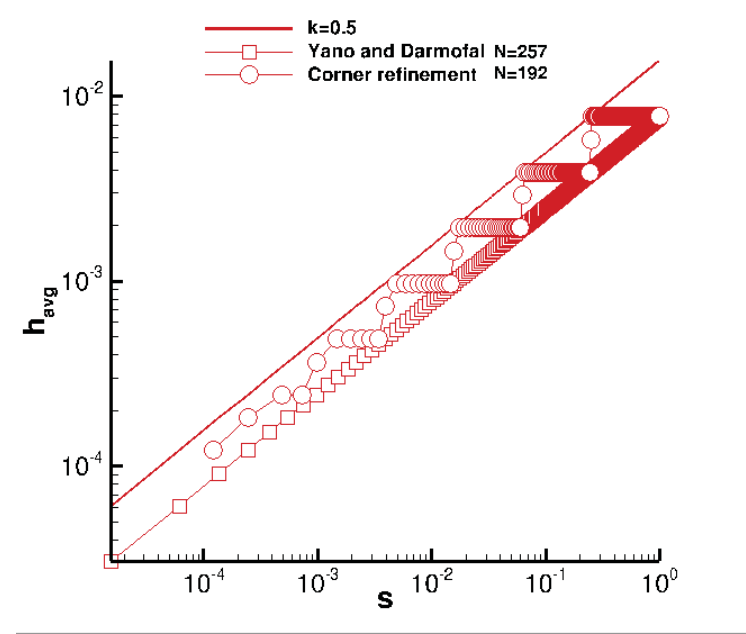

(a) Corner-refinement grids with $n=2$ and optimal grids with $k=0.5$;

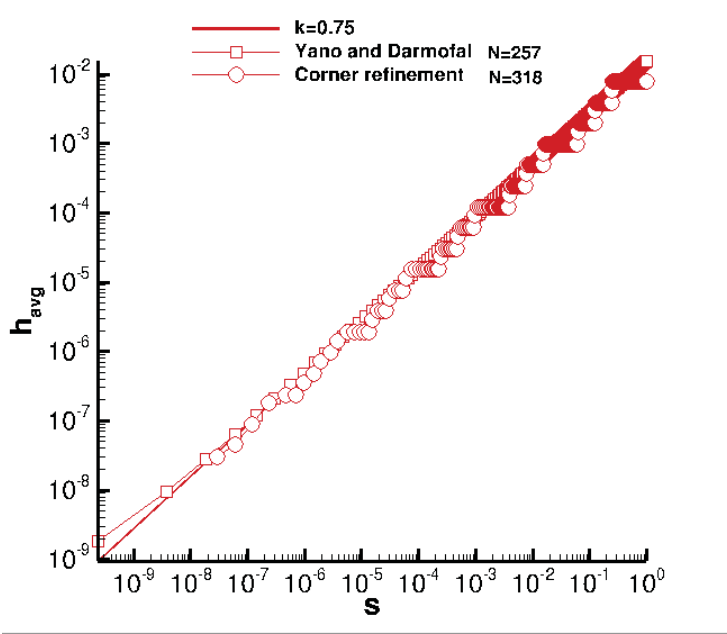

(b) Corner-refinement grids with $n=4$ and optimal grids with $k=0.75$.

Figure 69. Averaged mesh spacing on corner-refinement and optimal grids at equivalent gradation and comparable number of nodes; $s=1-x$. 